NASA Technical Memorandum 104564

\title{
Modeling Radiation Forces Acting on TOPEX/Poseidon for Precision Orbit Determination
}

\author{
J.A. Marshall \\ NASA Goddard Space Flight Center \\ Greenbelt, Maryland
}

S.B. Luthcke

Hughes STX Corporation

Lanham, Maryland

P.G. Antreasian and G.W. Rosborough

University of Colorado

Boulder, Colorado

\section{N/SA}

National Aeronautics and

Space Administration

Goddard Space Flight Center

Greenbelt, Maryland 20771 



\section{PREFACE}

Geodetic satellites such as GEOSAT, SPOT, ERS-1, and TOPEX/Poseidon require accurate orbital computations to support the scientific data they collect. Until recently, gravity field mismodeling was the major source of error in precise orbit definition. However, with recent improvements in these models, accurate modeling of the nonconservative forces has become a significant concern. In fact, the TOPEX/Poseidon mission requirements dictate that the mismodeling of the nonconservative forces of solar radiation, Earth albedo and infrared re-radiation, and spacecraft thermal imbalances produce in combination no more than a $6-\mathrm{cm}$ radial rms error over a 10-day period. This requires the development of nonconservative force models which take the satellite's complex geometry, attitude, and surface properties into account. Specifically, for TOPEX/Poseidon, a "box-wing" satellite form has been investigated. This approach models the satellite as the combination of flat plates arranged in the shape of a box and a connected solar array. The nonconservative forces acting on each of the eight surfaces are computed independently, yielding vector accelerations which are summed to compute the total aggregate effect on the satellite center-of-mass. Parameters associated with each flat plate are adjusted to obtain a better representation of the satellite acceleration history. In order to test the validity of this concept, "micro-models" based on finite element analysis of the TOPEX/Poseidon spacecraft have been used to generate acceleration histories in a wide variety of orbit orientations. These accelerations profiles are compared to those from the "box-wing" model. Tests indicate that modeling solar radiation pressure acceleration is relatively straightforward. However, the thermal imbalance modeling is made much more complicated given the satellite's complex attitude control law and its relation to the predicted temperature history for each surface. The results of these simulations and their implication on the ability to precisely model the TOPEX/Poseidon orbit will be discussed. 


\section{INTRODUCTION}

\section{Mission/Science Overview}

The seasonal variations of the world's ocean circulations, and how they influence the Earth's climate will soon be investigated with a high-precision Earth orbiting altimetric satellite. This project, referred to as the Ocean TOPography EXperiment/Poseidon Mission (T/P), is a joint venture between the U.S.'s National Aeronautics and Space Administration (NASA) and the French Centre National d'Etudes Spatiales (CNES). The TOPEX/Poseidon spacecraft will be equipped with two radar altimeters (1 US, and 1 French), which will measure the ocean surface topography.

By measuring the height of a satellite above the ocean surface with the use of a radar altimeter and subtracting the satelite's height in geocentric coordinates, the sea surface topography in geocentric coordinates is calculated. Subtracting the geoid height and accounting for Earth/ocean tides yields a measure of the sub-satellite ocean dynamic topography. Knowledge of the dynamic topography over time is very important in that it consists mainly of surface geostrophic currents and ocean thermal responses which drive global weather patterns and their changes.

The T/P spacecraft will orbit the Earth at an altitude of $1336 \mathrm{~km}$, inclination of $66^{\circ}$ and with nearly zero eccentricity. The period of the orbit is 1.87 hours and its groundtrace will repeat every 10 days in a "frozen" orbit. T/P is expected to be launched in July of 1992 using the European Space Agency's Ariane launch vehicle.

\section{Precision Orbit Determination}

Since the orbit of T/P will provide the reference frame from which the altimeter measurements are taken, any error in determining the satellite's position will affect the accuracy of the direct interpretation of the altimetric measurements. In order to obtain direct measurements of dynamic topography to the degree of accuracy that is required by the scientific community for oceanographic studies, the orbit of T/P must be determined over contiguous 10 day periods to within $13 \mathrm{~cm}$ RMS accuracy in the radial component with less than $5 \mathrm{~cm}$ RMS geographically correlated error [Stewart et al., 1986]. Orbit determination of this accuracy has never before been undertaken or achieved for a satellite at T/P's altitude.

The common method for computing the radiation pressure upon orbiting satellites with the orbit determination software has been to ignore these rotating, attitude controlled, geometrically complex shapes and treat the satellite form as a symmetrically perfect and 
rotationally invariant sphere, or so-called "cannonball." A typical cannonball radiationpressure model had a constant projected area with respect to the radiation source, and empirical scaling factors were commonly adjusted to improve the orbital fit to the tracking data. In addition, the thermal emission of the spacecraft itself was ignored. These approaches are not adequate to meet the required $6 \mathrm{~cm}$ radial $\mathrm{rms}$ error budget for modeling the radiation forces on T/P over a 10 day period. After considerable analysis of all surface force contributions, resultant models to be used in T/P orbit determination have been derived and are presented herein.

\section{Radiation Forces}

At the $1336 \mathrm{~km}$ altitude, solar radiation is the greatest nonconservative force acting on the spacecraft. In addition to direct solar radiation from the Sun, the Earth's albedo and infrared (IR) emissions, along with infrared emissions from the spacecraft itself will perturb the orbital motion. These effects are described below.

\section{Solar Radiation}

The Sun emits a nearly constant amount of photons per unit of time, varying less than $0.2 \%$ [Kive/son, 1986]. At a mean distance of 1 A.U., this time rate of flow of radiant energy per unit area, known as the solar constant or solar irradiance [Wilson and Hudson, 1988], is

$$
G_{\odot}=1367.7 \mathrm{~W} / \mathrm{m}^{2}
$$

Since the Earth's orbit is eccentric, the solar radiation flux received by the Earth varies by $\pm 40.6 \mathrm{~W} / \mathrm{m}^{2}$ throughout its orbit around the Sun. The force produced by this radiation is by far the largest of the radiative effects. Also, for T/P, it is the largest nongravitational force acting on the satellite.

\section{Earth Albedo and Infrared Radiation}

A fraction of the solar radiation is instantly reflected off the land-water-snow-iceatmosphere system as shortwave energy and the remainder is absorbed by the Earth. This absorbed energy is later emitted as longwave infrared radiation.

The development given by Knocke et al., 1988 for modeling the radiation force due to Earth albedo and infrared emission has been adopted for this study. For the case of albedo, the amount of radiation received by a satellite due to an elemental Earth surface area $d A_{\oplus}$, will be, 


$$
G_{\oplus}=\frac{a G_{\odot}}{\pi r^{2}} \cos \theta_{\odot} \cos \gamma d A_{\oplus}
$$

where $\theta_{\odot}$ is the angle between the unit normal of the elemental Earth surface area and the direction to the Sun, $\gamma$ is the angle between the Earth surface unit normal and the direction to the satellite, $r$ is the distance from the Earth surface element to the satellite, and $a$ is the albedo coefficient of the surface element. This model assumes that the Earth reflects and emits most of its energy in a diffuse manner and, therefore, each elemental Earth area radiates constant flux. In general, the $a$ varies over the entire irradiated portion of the Earth. For this application, a longitudinally averaged model of albedo, as specified by Knocke et al. [1988] has been used. The average value of the albedo coefficient is 0.34, which means the reflecting Earth surface elements radiate, on average, $465 \mathrm{~W} / \mathrm{m}^{2}$.

The infrared radiant energy flux density received by the spacecraft from an elemental Earth area $d A_{\infty}$ at a distance, $r$, is

$$
G_{\oplus_{\varepsilon}}=\frac{\epsilon G_{\odot}}{4 \pi r^{2}} \cos \gamma d A_{\oplus}
$$

where $\epsilon$ is the emissivity of the surface element. As with the albedo, a simple latitudinal model is used for the emissivities. The average emissivity of the Earth is 0.68 , which results in the surface elements radiating, on average, $232 \mathrm{~W} / \mathrm{m}^{2}$.

Spacecraft Radiation

In addition to the radiation forces produced by the direct effects of incident radiation, a heated surface produces a thermal force due to its emission of infrared energy. A spacecraft surface with a temperature of $T$ will emit longwave radiation at the level

$$
G_{s / c}=\operatorname{eot} T^{4}
$$

where $\sigma$ is the Stefan-Boltzmann constant $\left(5.670 \times 10^{-8} \mathrm{~W} / \mathrm{m}^{2} \mathrm{~K}^{4}\right)$ and $\epsilon$ is the emissivity of the surface. As the temperature of the surface changes throughout the orbit (especially due to eclipsing) the magnitude of the thermal force can change substantially. The surface itself changes because of the hostile orbital environment, and these effects must also be considered. 


\section{TOPEX/Poseidon Spacecraft}

The T/P spacecraft will fly in a near-circular orbit at an altitude of $1336 \mathrm{~km}$ and an inclination of 66 degrees. The eccentricity is controlled to maintain the argument of perigee at 90 degrees, producing a "frozen" orbital mission. This frozen orbit configuration minimizes height variations (with respect to the ellipsoid) and is very favorable for altimetric missions. Table 1 summarizes the orbital characteristics.

Table 1. Orbital Parameters

\begin{tabular}{|l|c|r|}
\hline Semi-major axis & $a$ & $7714 \mathrm{~km}$ \\
\hline Eccentricity & $\theta$ & 0.00 \\
\hline Inclination & $i$ & $66^{\circ}$ \\
\hline Argument of Perigee & $\omega$ & $90^{\circ}$ \\
\hline Altitude & $h$ & $1336.00 \mathrm{~km}$ \\
\hline Nodal Precession Rate & $\Omega$ & $-2.31 \mathrm{deg} / \mathrm{day}$ \\
\hline Period & $P$ & $112 \mathrm{~min}$ \\
\hline
\end{tabular}

The spacecraft consists of an instrument module, propulsion module and a single solar array. A representation of the spacecraft is shown in Figure 1a and the corresponding approximate projected areas of the spacecraft are summarized in Table 2. Note that there is a significant variation in the projected area-to-mass ratio depending on the view direction and that the spacecraft exhibits considerable asymmetries.

Table 2. Projected Area Properties

\begin{tabular}{||l|l|r|}
\hline X projected s/c body area & $A_{x}$ & $4.70 \mathrm{~m}^{2}$ \\
\hline Y projected s/c body area & $A_{y}$ & $8.18 \mathrm{~m}^{2}$ \\
\hline$Z$ projected s/c body area & $A_{z}$ & $8.30 \mathrm{~m}^{2}$ \\
\hline Solar array area & $A_{s}$ & $25.5 \mathrm{~m}^{2}$ \\
\hline Spacecraft mass & $m$ & $2500.0 \mathrm{~kg}$ \\
\hline Max. proj. area-to-mass ratio & & $0.140 \mathrm{~cm}^{2} / \mathrm{g}$ \\
\hline Min. proj. area-to-mass ratio & & $0.019 \mathrm{~cm}^{2} / \mathrm{g}$ \\
\hline
\end{tabular}




\section{Attitude/Yaw Steering}

The TOPEX/Poseidon spacecraft has an intricate attitude control system because of its large, single-axis gimbal solar array. Perfect solar pointing (Sun incidence vector normal to the solar array) requires the spacecraft to yaw about its Earth-pointing Z-axis at rates that exceed the capabilities of the attitude control system. Therefore, Fairchild Space Company (FSC) designed a sinusoidal yaw command that achieves near-perfect solar pointing while remaining within attitude control system limits [Perrygo, 1987]. The algorithm is based on the coordinate systems shown in Figures $1 \mathrm{~b}$ and 2 . The spacecraft body-fixed system origin is within the vehicle body, although not at the center of mass, with the positive $Y$-axis pointed opposite of the solar array axis, the positive $Z$-axis directed to the Earth nadir, and the positive $X$-axis orthogonal to the $Y$ and $Z$ axes to complete the right-handed system. The inertial system is centered at the geocenter with the $X_{0}$-axis normal to the satellite orbit plane, the $Z_{0}$-axis pointed in the direction of the Sun as projected into the orbit plane, and the $Y_{0}$-axis normal to these axes. As shown in Figure 2, $B^{\prime}$ refers to the angle between the Sun vector and the orbit plane and $\Omega$ is the orbit angle, measured from the $Y_{0}$-axis.

The actual yaw angle, $\psi$, of the spacecraft is rotated positively from the $X_{p}$-axis (the alongtrack direction) about the $Z$-axis and is determined from the $B^{\prime}$ and $\Omega$. The multiple yaw steering algorithms the satellite follows during its orbit are summarized in Table 3 [Zimbelman, 1989]. The B' and $\Omega$ yaw steering mode boundary values specified are the nominal values selected for the mission and may be changed at any time by ground controllers. 
Table 3. TOPEX/Poseidon Yaw Steering Modes

\begin{tabular}{|c|c|c|c|}
\hline $\begin{array}{l}\text { Yaw Steering } \\
\text { Mode }\end{array}$ & $B^{\prime}$ Region & \& Reglon & Yaw Algorlthm \\
\hline \multirow{4}{*}{ Flxed } & $+0.1^{\circ} \leq B^{\prime} \leq+15^{\circ}$ & All & $\boldsymbol{\Psi}=0^{\circ}$ \\
\hline & $-15^{\circ} \leq \mathrm{B}^{\prime} \leq-0.1^{\circ}$ & All & $\Psi=180^{\circ}$ \\
\hline & $\mathrm{B}^{\prime} \geq+80^{\circ}$ & All & $\boldsymbol{\Psi}=90^{\circ}$ \\
\hline & $B^{\prime} \leq-80^{\circ}$ & All & $\Psi=-90^{\circ}$ \\
\hline \multirow{2}{*}{ Sinusoldal } & $\mathrm{B}^{\prime}>+15^{\circ}$ & All & $\boldsymbol{\Psi}=90^{\circ}+\left(90^{\circ}-\mathrm{B}^{\prime}\right) \cos \Omega$ \\
\hline & $\mathbf{B}^{\prime}<-15^{\circ}$ & All & $\Psi=-90^{\circ}-\left(90^{\circ}+B^{\prime}\right) \cos \theta$ \\
\hline \multirow{2}{*}{ Ramp Up } & $\mathbf{B}^{\prime}=+15^{\circ}$ & $90^{\circ} \leq \Omega \leq 180^{\circ}$ & $\Psi=B^{\prime} \cos ^{2} \Omega$ \\
\hline & $B^{\prime}=-15^{\circ}$ & $270^{\circ} \leq \Omega \leq 360^{\circ}$ & $\boldsymbol{\Psi}=-\mathrm{B}^{\prime} \cos ^{2} \Omega-180^{\circ}$ \\
\hline \multirow{2}{*}{ Ramp Down } & $B^{\prime}=+15^{\circ}$ & $180^{\circ} \leq \Omega \leq 270^{\circ}$ & $\Psi=B^{\prime} \cos ^{2} \Omega$ \\
\hline & $B^{\prime}=-15^{\circ}$ & $0^{\circ} \leq \Omega \leq 90^{\circ}$ & $\Psi=-B^{\prime} \cos ^{2} \Omega-180^{\circ}$ \\
\hline \multirow{2}{*}{ Fllp } & $0^{\circ} \leq B^{\prime}<0.1^{\circ}$ & $-180^{\circ} \leq \Omega \leq 0^{\circ}$ & $\Psi=-90^{\circ}[1+\cos \Omega]$ \\
\hline & $-0.1^{\circ} \leq \mathbf{B}^{\prime}<0^{\circ}$ & $-180^{\circ} \leq \Omega \leq 0^{\circ}$ & $\Psi=-90^{\circ}[1-\cos \Omega]$ \\
\hline
\end{tabular}


As Table 3 suggests, the yaw angle computation algorithm is extremely intricate at the yaw regime boundaries. When the on-board computer indicates that the spacecraft has crossed a B' limit, it sets a flag to initiate the steering mode when it next crosses into the appropriate orbit angle transition regime. If the satellite crosses a B' limit while in its corresponding orbit angle transition area, it will wait an entire revolution to commence yaw mode changes.

The solar array pitch angle is defined as,

$$
\begin{aligned}
0^{\circ}<B^{\prime}<15^{\circ} & \gamma=0 \\
-15^{\circ}<B^{\prime}<0^{\circ} & \gamma=\Pi-Q \\
B 1 \geq 15^{\circ} & \gamma=\arctan \left(\frac{\sin \Omega \cos \beta^{\prime}}{\cos Q \cos \beta^{\prime} \cos \Psi-\sin \Psi \sin \beta^{\prime}}\right)
\end{aligned}
$$

where the pitch angle, $\gamma$, rotates positively from the spacecraft $X$-axis about the $Y$-axis to orient the cells toward the Sun for optimum Sun pointing.

\section{Model Development}

The first step in a detailed analysis of the radiation forces acting on T/P was to accurately compute the radiation forces due to the Sun, Earth albedo, Earth infrared, and spacecraft thermal emissions upon T/P with the use of a finite element model of the spacecraft. This investigation generated what is referred to as the "micro-models"; a thorough explanation of this modeling effort is given by Rosborough and Antreasian [1991]. Since a precise thermal and radiative model of a spacecraft is necessarily computationally intensive, this micro-model, which served as a "truth" model, was computed offline. A relatively simple and less computationally intensive model, or macro-model, more suitable for precision orbit computations, was devised and tested to emulate the micro-model accelerations. This model was introduced in Marshall et al., 1991. A graphical representation of this development is shown in Figure 1.

\section{Micro-Models}

A radiation force is produced on a spacecraft surface if that spacecraft surface absorbs, reflects and/or emits radiant energy. Hence, forces will be generated by incident radiant energy from the Sun or Earth falling on a surface and by radiant energy emission from the surface. The detailed evaluation of the radiation forces is dependent upon accurately defining the flux that is incident on the various spacecraft surfaces and the resulting 
interaction (absorption, reflection). The evaluation of these processes has been carried out using industry standard software by correctly orienting the spacecraft for a given orbital location and determining the incident fluxes on hundreds of unique elements comprising T/P [Rosborough and Antreasian, 1991]. Once the fluxes on each surface are determined for all locations around a single orbit, their transient temperatures were determined. These fluxes and temperatures are then converted to resultant forces based on the surface properties and orientation of each element. The resultant forces were then summed to get the total force acting on the spacecraft at each point in the orbit. A wide array of orbit orientations were evaluated for each force and acceleration histories in the radial, along-track, and cross-track directions were generated. The direct solar radiation, Earth albedo and IR micro-models were evaluated at B' increments of 4 degrees and $\mathbf{3 0}$ points about the orbit for each B'. The thermal imbalance acceleration on the spacecraft was computed at B' increments of 8 degrees and 72 orbit angles for each B'. Consideration also has to be given to the reflective and absorptive properties of the surfaces which will degrade with time due to the inhospitable orbit environment.

A few assumptions have been made in computing the T/P micro-models. These include:

- Circular orbit
- $\quad$ Comp and flip yaw steering modes ignored
- Diffuse reflection and emission obey Lambert's cosine law
for angular distribution of energy
- The contribution of the force due to the specular
surface-to-surface reflections is negligible
- Cylindrical Earth shadow model
- Constant solar, Earth albedo, and Earth IR fluxes
- $\quad$ components of the spacecraft reflect the same percentage of
reflectenergy specularly

The last assumption is due to data not being currently available for each surface coating, but will be incorporated later. Philosophically, the circular orbit and simplistic yaw steering provide a useful intermediary "orbit" on which to derive the response of the spacecraft to the indicated forces.

\section{Macro-Models}

The micro-models yield the best available detailed force model history of the spacecraft. However, their computational requirements are excessive, taking as much as 1 day to process a single orbital revolution on a typical computer workstation. Therefore, a more simplistic approach which still mimics the spacecraft's reaction to radiative forces at a 
satisfactory level has been adopted. This concept is based on approximating the satellite shape with a combination of flat plates. The nonconservative forces acting on each of the composite surfaces are computed independently. All plate interaction effects, such as shadowing, reflection, and conduction are ignored. This yields vector accelerations which are summed to compute the total effect on the spacecraft center-of-mass. The algorithm includes the ability to adjust aggregate parameters associated with each flat plate to obtain a better fit to the actual satellite acceleration history based on orbit errors sensed from laser tracking data and from telemetered satellite on-orbit temperatures. For $T / P$, a box-wing shape was chosen with the plates aligned along the satellite body-fixed coordinate system (Figure 1).

During development, every attempt has been made to keep the macro-models as simple as possible while still meeting T/P mission requirements and avoiding telemetry dependence. These concepts are often in conflict and, as in the case of the spacecraft radiation model, simplicity yielded to the needs of meeting modeling requirements.

\section{Solar and Earth Radiation}

Solar, albedo, and infrared emissions are the three external radiative fluxes acting on a spacecraft. The radiation pressure acting on a flat plate can be computed using the following equation [Milani et al., 1987], assuming a Lambertian diffusion,

$$
\Gamma=-\frac{G A \cos \theta}{M C}[2(\delta / 3+\rho \cos \theta) \vec{n}+(1-\rho) \vec{s}]
$$

where

$\begin{array}{lll}\Gamma & \equiv \text { radiation pressure acceleration on the flat plate } \\ \mathbf{A} & \equiv \text { surface area of the flat plate } \\ \boldsymbol{\theta} & \equiv \text { angle between surface normal and Sun source vectors } \\ \mathbf{G} & \equiv \text { radiation flux from source } \\ \mathbf{n} & \equiv \text { surface normal vector } \\ \mathbf{S} & \equiv \text { source incidence vector } \\ \mathbf{P} & \mathbf{3} \text { specular reflectivity (percentage of total incoming radiation) } \\ \delta & \equiv \text { diffusive reflectivity (percentage of total incoming radiation) } \\ \mathbf{M} & \equiv \text { satellite mass } \\ \mathbf{C} & \equiv \text { speed of light }\end{array}$

The adjustable parameters are area and specular and diffusive reflectivity. These parameters are averaged values which represent the consolidation of the spacecraft's complex shapes and material properties into a single, homogeneous flat plate. 
The albedo and infrared accelerations use a similar acceleration equation as the solar radiation. However, the flux magnitude is different. Also, the source vector is the Earth grid spot-to-satellite vector rather than the solar incidence vector. The spot definition and location are defined by Knocke et al., [1988]. Note that this model is not self-shadowing. The total albedo/infrared acceleration can be expressed as:

$$
\Gamma=-\sum_{1}^{N} \sum_{f}^{19} \frac{G_{f} A_{1} \cos \theta_{1 j}}{M C}\left[2\left(\delta_{1} / 3+p_{1} \cos \theta_{1 j}\right) \vec{n}_{1}+\left(1-\rho_{1}\right) \vec{s}_{f}\right]
$$

where

$$
\begin{array}{lll}
\mathbf{i} & =\text { plate of interest } \\
\mathbf{j} & \mathbf{\equiv} \text { Earth spot of interest } \\
\mathrm{n} & \equiv \text { total number of plates }
\end{array}
$$

\section{Spacecraft Radiation}

Two separate types of fluxes affect the flat plate temperatures: internal and external. Internally, the equipment dissipates radiation which serves to heat the satellite surfaces. Externally, the solar radiation, albedo, and infrared fluxes cause surface heating. The force exerted on a surface due to thermal emission, assuming a Lambertian diffusion function, can be expressed as:

$$
\vec{F}=-\frac{2 A \sigma}{3 C} e T^{4} \vec{n}
$$

where

$$
\begin{array}{lll}
\epsilon & \equiv \text { emissivity } \\
\sigma & \equiv \text { Stefan-Boltzmann constant }\left(5.67 \mathrm{E}-08 \mathrm{~W} / \mathrm{m}^{2} / \mathrm{K}^{4}\right) \\
\mathrm{A} & \equiv \text { surface area } \\
\mathrm{T} & \equiv \text { temperature }(\text { Kelvin }) \\
\mathrm{C} & \equiv \text { speed of light }(\mathrm{m} / \mathrm{s}) \\
\mathrm{n} & \equiv \text { surface normal vector }
\end{array}
$$

The temperature history algorithm, however, is not as clearly defined. One must take into account the complexities of (1)occultation effects, (2)oblique illumination, and (3) the spacecraft's thermal inertia, without losing sight of the need for simplicity and generality.

The temperature for a surface exposed to sunlight is modelled as

$$
T=a+c * \cos \left(\frac{\theta}{x}\right)\left[1-\exp \left(-\frac{t_{1}}{f}\right)\right]
$$


and while in shadow as

$$
T=a+c \exp \left(-\frac{t_{2}+s_{2}}{d}\right)
$$

where

$$
s_{2}=-d l n\left(\cos \left(\frac{\theta_{\text {shd }}}{x}\right)\left[1-\exp \left(-\frac{t_{1}}{f}\right)\right)\right)
$$

and

$$
\begin{array}{lll}
a & \equiv \text { cold equilibrium surface temperature } \\
c & \equiv \text { delta temp. between cold and hot equilibrium } \\
d & \equiv \text { transition time from hot to cold equilibrium temp. } \\
f \quad \equiv \text { transition time from cold to hot equilibrium temp. } \\
x \quad \equiv \text { rotation rate/thermal inertia constant } \\
t_{1} \equiv \text { time since shadow exit } \\
t_{2} \equiv \text { time since shadow entry } \\
s_{1} \quad \equiv \text { shift parameter to ensure continuity } \\
s_{2} \quad \equiv \text { shift parameter to ensure continuity } \\
\theta \quad \equiv \text { angle between surface normal and solar incidence } \\
\theta_{\text {shd }} \equiv \text { angle between normal and Sun vectors at shadow entry } \\
\theta_{\text {sun }} \equiv \text { angle between normal and Sun vectors at shadow exit }
\end{array}
$$

The adjustable parameters are area, emissivity, and all five temperature terms $(a, c, d, f, x)$.

An explanation of these equations is appropriate. First, note that solar radiation is the only direct effect influencing the temperature. That is to say, the $\theta$ angle and the time parameters are based only on solar illumination and neglect the albedo and IR effects. However, albedo and IR do indirectly influence the apriori values of all the adjustable temperature parameters. A plate's orientation with respect to the Sun dictates which temperature algorithm to use. The cosine term in the sunlight equation allows for the fact that an obliquely illuminated plate will have a lower temperature than one perpendicular to the sun vector. This is illustrated in Figure 3 which shows the temperature profile at $\mathrm{B}^{\prime}=0^{\circ}$ for a node on the $\mathrm{X}+$ plate as predicted by the micro-model. At an orbit angle of $330^{\circ}$, the face enters sunlight and warms rapidly, reaching maximum exposure at $\Omega=$ $0^{\circ}$. The cooling from this point until the plate enters shadow at $\Omega=90^{\circ}$ results from the decreasing projected area as it rotates with respect to the sun. In contrast, a plate's cooling pattern, when occulted by either the Earth or the spacecraft, is independent of 
the sun position and, therefore, no such allowance needs to be made. The $x$ parameter in the denominator of the cosine term accounts for the fact that the temperature is not directly proportional to the rate at which spacecraft rotation moves a plate from direct to oblique illumination. Without $x$, there is no delay in reaching the cold equilibrium value as the plate enters shadow. For example, Figure 3 shows that at shadow entry, $\Omega=90^{\circ}$, the plate's temperature is not at its coldest point.

The exponential term addresses the occultation transition effects. As a face enters or leaves shadow, its temperature can be approximated by an exponential curve as shown in Figure 4, depicting the $B^{\prime}=0^{\circ}$ micro-model temperature profile for a node on the $X$ face. A different time constant ( $d$ or $f$ ) is applied depending on whether the surface is heating or cooling.

Finally, a shift term is introduced to ensure continuity in the transition from the sunlight to shadow temperature equation. This assumes that the plate will reach its cold equilibrium temperature in shadow before heating begins. Given that this assumption is not true, a different set of shift parameters must be established. For example, during sinusoidal yaw the $X$ - face of $T / P$ is occulted only by the Earth. As $B^{\prime}$ increases, this shadow time gradually decreases. Therefore, the plate will always reach its hot equilibrium temperature and not necessarily reach its cold equilibrium temperature as exhibited in Figure 5. In this case the following temperature history algorithms are used: Sunlight:

$$
T=a+c * \cos \left(\frac{\theta}{x}\right)\left[1-\exp \left(\frac{-\left(t_{1}+s_{1}\right)}{f_{1}}\right)\right]
$$

where

$$
s_{1}=-f \ln \left[1-\exp \left(-\frac{t_{2}}{d}\right)\left(\frac{\cos \theta_{\text {shd }}}{\cos \theta_{\text {sun }}}\right)\right]
$$

Shadow:

$$
T=a+c * \cos \exp \left(-\frac{\left(t_{2}+s_{2}\right)}{d}\right)
$$

where

$$
s_{2}=-d \ln \cos \left(\frac{\cos \theta_{\text {shd }}}{x}\right)
$$


More simplistic representations have been tried with varying success. However, they all fail to replicate certain thermal behaviors exhibited by the micro-model. A model of this complexity is necessary to meet T/P mission requirements and, therefore, this is the chosen parameterization for T/P modeling.

\section{Results}

In order to test the validity of these macro-models, a comparison has been performed between the acceleration histories predicted by the macro and micro-models. A Bayesian least squares estimation procedure has been used to tailor the adjustable macro-model parameters to better fit the micro-model generated acceleration histories for the solar radiation and the thermal imbalance nonconservative forces as outlined by Marshall et al., [1991]. Apriori values with realistic uncertainties served to constrain some certain highly correlated parameters that could not be recovered independently. Specifically, the solar array parameters were not adjusted since their properties are relatively well known and they do not represent an average of many smaller surfaces of varying characteristics. Also, to ensure realistic temperature values, the equilibrium temperatures, $a$ and $c$, were constrained so as not to stray more than a few degrees from the values predicted by the micro-model. Parameters associated with the $X+, Y+, Y-$, and SA- faces exhibited the weakest recovery due to their limited solar exposure. To date, the solar radiation and thermal imbalance forces have been fit independently and appropriate parameters recovered. Therefore, nonphysical properties could result when the terms are considered jointly. With the delivery of the "as-built" spacecraft models, new micro-models will be generated and a more aggressive and thorough macro-model parameter recovery will be undertaken. These adjusted values will be adopted as nominal values in the actual precision orbit determination computations.

All of the macro-models described herein have been implemented in the precision orbit determination software package at NASAGSFC, GEODYN [Putney et al., 1991]. The results presented in this paper use GEODYN and the T/P macro-models to simulate a 10 day T/P orbit. Table 4 describes the individual plate normal vectors in the satellite bodyfixed system used in the "box-wing" representation of the T/P spacecraft. Tables 5 and 6 show the actual macro-model parameter values used in this testing as derived from the least- squares adjustment outlined previously. It is important to note that a constant value of .34 for Earth albedo and .68 for Earth emissivity were used to represent the Earth albedo and IR forces. These values were used as constants to be consistent with the micro-model generation. 
TABLE 4. Macro-Model Plate Normal Vectors in the Spacecraft Body-Fixed System

\begin{tabular}{|c|c|c|c|}
\hline PLATE & $X$ & $Y$ & $Z$ \\
\hline$X+$ & 1.0 & 0.0 & 0.0 \\
\hline$X-$ & -1.0 & 0.0 & 0.0 \\
\hline$Y+$ & 0.0 & 1.0 & 0.0 \\
\hline$Y-$ & 0.0 & -1.0 & 0.0 \\
\hline$Z+$ & 0.0 & 0.0 & 1.0 \\
\hline$Z-$ & 0.0 & 0.0 & -1.0 \\
\hline SA+ & 1.0 & 0.0 & 0.0 \\
\hline SA- & -1.0 & 0.0 & 0.0 \\
\hline
\end{tabular}

Table 5. Plate Optical and Thermal Characteristics for Tuned Thermal Model

\begin{tabular}{|c|c|c|c|c|c|c|c|c|}
\hline & $X+$ & $X-$ & $Y+$ & $Y-$ & $Z+$ & $Z-$ & SA+ & SA- \\
\hline \hline Area & 4.71 & 4.71 & 8.18 & 8.18 & 8.32 & 8.32 & 25.50 & 25.50 \\
\hline Specular Ref. & .201 & .244 & .886 & .782 & .239 & .275 & 0.05 & 0.17 \\
\hline Diffuse Ref. & .375 & .386 & .302 & .339 & .390 & .363 & 0.22 & 0.66 \\
\hline Emissivity & .769 & .995 & .873 & .714 & .770 & .746 & 0.87 & 0.88 \\
\hline Temp. A & 181 & 168 & 191 & 190 & 240 & 103 & 236 & 234 \\
\hline Temp. C & 233 & 178 & 18 & 63 & 98.5 & 125 & 110 & 96 \\
\hline Time D & 621 & 282 & 759 & 426 & 519 & 680 & 805 & 806 \\
\hline Time F & 111 & 120 & 624 & 487 & 767 & 413 & 828 & 866 \\
\hline ThetaX & 1.25 & 1.00 & 1.05 & 1.00 & 1.06 & 1.15 & 1.00 & 1.00 \\
\hline
\end{tabular}


Table 6. Plate Optical and Thermal Characteristics for Tuned Solar Radiation Pressure Model.

\begin{tabular}{|c|c|c|c|c|c|c|c|c|}
\hline & $X+$ & $X-$ & $Y+$ & $Y-$ & $Z+$ & $Z-$ & SA + & SA- \\
\hline \hline Area & 3.74 & 3.77 & 8.27 & 8.07 & 8.67 & 8.44 & 21.4 & 21.44 \\
\hline Specular Ref. & .201 & .244 & .886 & .782 & .239 & .275 & 0.05 & 0.17 \\
\hline Diffuse Ref. & .375 & .386 & .302 & .339 & .390 & .363 & 0.22 & 0.66 \\
\hline Emissivity & .769 & .995 & .873 & .714 & .770 & .746 & 0.87 & 0.88 \\
\hline Temp. A & 181 & 168 & 191 & 190 & 240 & 103 & 236 & 234 \\
\hline Temp. C & 233 & 178 & 18 & 63 & 98.5 & 125 & 110 & 96 \\
\hline Time D & 621 & 282 & 759 & 426 & 519 & 680 & 805 & 806 \\
\hline Time F & 111 & 120 & 624 & 487 & 767 & 413 & 828 & 866 \\
\hline ThetaX & 1.25 & 1.00 & 1.05 & 1.00 & 1.06 & 1.15 & 1.00 & 1.00 \\
\hline
\end{tabular}

The most important of all the T/P specific models added to the orbit determination software is the attitude control logic because all the subsequent nonconservative force models rely on the correct orientation of the individual plates. Therefore, substantial effort was expended checking this logic. The results not only validate the code but provide penetrating insight into the T/P force modeling.

Figures 6-8 demonstrate the behavior of the B', yaw, and solar array pitch angles for a spacecraft in a simulated T/P orbit over a 1-year period. These parameters were sampled at a rate considerably larger than the orbital period. Therefore, these plots do not attempt to show physical dependence on orbital period or ramping region data. Instead they demonstrate parameter amplitude and trends. Figure 6 exhibits the variations in B' over 1 year. During this period, B' variations complete a full cycle. The absolute value of B' never exceeds $89.5^{\circ}$ since the orbital inclination is $66^{\circ}$ and the ecliptic angle is $23.5^{\circ}$. Figure 7 demonstrates that all the yaw steering regimes are exercised and that the model adheres to the logic specified in Table 3. Note that the yaw angle transitions directly from $0^{\circ}$ to $-180^{\circ}$ instead of following the dictated flip algorithm as the spacecraft crosses $B^{\prime}=0^{\circ}$. It is assumed that tracking data taken during this regime is ignored and that its effect on the nonconservative force modeling would be minimal. In Figure 8 , note that the solar array pitch angle (SAPA) oscillates about $0^{\circ}$ or $180^{\circ}$ depending upon the yaw steering algorithm. In fixed yaw, where the SAPA is directly related to the orbit angle, 
one sees the largest variations in amplitude. In sinusoidal yaw, where the intent is to keep the solar array pointing perpendicular to the Sun, the SAPA amplitude gradually decreases with increasing B'. Almost no variation in SAPA is seen at high B'.

Figures 9-15 show the projected area of each face in the Sun direction as a function of $B$ ' and orbit angle. These plots, more than anything else, lend to the understanding of the complexities of the T/P radiative nonconservative forces. Beginning with the $X+$ face in Figure 9, this face is illuminated as the spacecraft leaves the Earth occultation boundary until it reaches a $90^{\circ}$ orbit angle. Once the satellite enters sinusoidal yaw, this plate is never again illuminated. This is done to keep the propulsion module, or $\mathrm{X}$ - face, in the sun direction and, therefore, keep the propellants warm, for as much of the time as possible, as shown in Figure 10. Note that the Earth occultation effect gradually decreases with increasing $B^{\prime}$. At $B^{\prime}>56^{\circ}$, the Earth's shadow no longer occults the $T / P$ orbit. Figures 11 and 12 demonstrate how little the $Y+$ and $Y$ - faces are illuminated. The $Y$ - face receives more sunlight on average than the $Y+$ as it is on the same side of the spacecraft as the solar array. Figure 13 exhibits the $Z+$ plate exposure. This nadirpointing face is unique in that for $B^{\prime}<56^{\circ}$ it is illuminated twice per orbit. From $0^{\circ}$ to $180^{\circ}$ orbit angle the face is rotated away from the sun while the spacecraft itself is illuminated. It then receives sunlight for the brief periods between Earth occultation and these $180^{\circ}$ and $360^{\circ}$ orbit angles. These periods of sunlight increase with increasing B'. Figure 14 shows the very symmetric illumination pattern of the Z- face. Note that, as with the Z+ face, the sun's rays become more glancing as $B$ ' increases and the projected area goes to nearly zero. Finally, Figure 15, showing the solar array projected area, clearly outlines the Earth occultation region. It is the largest and most stable, in terms of projected area, of all the plates. These visibility plots are the main drivers behind the radiative force model acceleration histories discussed in the following sections.

\section{Solar Radiation}

Solar radiation is the dominant radiative force acting on T/P. Its acceleration profile is driven by the large area of the solar array which tracks the Sun throughout the orbit. Plots of the micro-model solar radiation acceleration in the along-track, cross-track and radial directions are shown in Figures 16, 18, and 20. Data spacing is at 4-degree B' increments and 15 degree orbit angle increments. The " $U$ " shaped outline of the Earth occultation region is clearly visible. At $B^{\prime}=O^{\circ}$ the spacecraft is in fixed yaw and the Sun is edge on to the orbit plane. Therefore, the plots correctly display no cross-track acceleration, a sinusoidal signal in the radial direction, and a similar signal offset 90 degrees in phase in the along-track component. T/P is in a sinusoidal yaw at $B^{\prime}=40^{\circ}$ and the almost constant cross-track force drops to zero during occultation. The alongtrack and radial accelerations have smaller amplitudes but virtually the same shape as at 
$B^{\prime}=0^{\circ}$. At $B^{\prime}=88^{\circ}$, the spacecraft is in continuous sunlight, the sun is nearly perpendicular to the orbit plane, and the cross-track acceleration dominates.

As exhibited in Figures 17,19,and 21, the macro-model was exercised in GEODYN to generate data for comparison with the micro-model. Notice that the macro-model captures all of the features of the micro-model, even though the profiles change drastically throughout the different $B^{\prime}$ regimes. The residuals between the micro and macro-models in each of the three directions are displayed in Figures 22-24. The prominent residual spikes appearing around the Earth occultation boundary are caused by a different definition of this boundary's location in the micro and macro-models and, therefore, is not a problem since GEODYN calculates shadow entry and exit times very precisely. It is anticipated that the remaining residual exhibited in the along-track and radial residual plots will be greatly reduced with proper tuning of the solar array macromodel parameters.

\section{Earth Albedo}

The size of the Earth albedo acceleration on the T/P spacecraft is an order of magnitude smaller than that of the solar radiation pressure. Its signal is quite different from the solar radiation in that the solar array is not always oriented nearly perpendicular to the incident flux vector. The micro-model albedo acceleration history in the along-track, cross-track, and radial directions is shown in Figures 25,27 , and 29 . The accompanying macromodel and residual plots are shown in Figures $26,28,30$ and $31-33$, respectively. Note that the acceleration is dominated by the radial direction peak at $90^{\circ}$ orbit angle and $0^{\circ}$ B' (Figures 29,30). At this point the maximum spacecraft projected area (solar array and $\mathrm{Z}+$ face) is perpendicular to the albedo flux. This signal gradually fades as the orbit angle approaches $0^{\circ}$ or $180^{\circ}$ or as $B^{\prime}$ increases, and the solar array moves edge on to the incident flux. Similarly, the along-track acceleration has the greatest amplitude at low B'. The opposing humps in Figures 25 and 26 represent similar spacecraft maximum projected area configurations on either side of the $90^{\circ}$ orbit angle boundary. From $0^{\circ}$ to $90^{\circ}$, the albedo pushes the spacecraft, while from $90^{\circ}$ to $180^{\circ}$ it slows the spacecraft motion (Figures 25,26). The residual plots do not exhibit the same spiking at the occultation boundaries as seen in the solar radiation plots since there is virtually no albedo flux striking the spacecraft in this regime. It is important to remember two points when examining these residual plots. First, the micro-models were generated using a $B^{\prime}=10^{\circ}$ fixed to sinusoidal yaw transition boundary. The macro-models, however, used a $B^{\prime}=15^{\circ}$ boundary value. this leads to some of the signal seen in the residuals for this region. Second, the albedo and IR macro-models were never tuned to the micro-models. Therefore, a much better fit is anticipated in the future. 


\section{Earth IR}

The Earth IR acceleration is very similar to the albedo signal. However, it is not influenced by Earth occultation and has nearly constant flux throughout the orbit. Therefore, the accelerations exhibit a symmetry about $180^{\circ}$ orbit angle. The micro-model, macro-model, and residual acceleration plots are displayed in Figures 34-42. The narrow lip or shelf at low $B$ ' in the cross-track plots exists because, until the spacecraft transitions to sinusoidal yaw, there is virtually no cross-track signal. Also, observe that the largest magnitude along-track accelerations occur at low orbit angles in the macro-model (Figure 35) but at high orbit angles in the micro-model (Figure 34). The dips in the radial acceleration in the micro-model (Figure 38) are symmetric whereas they are not symmetric in the corresponding macro-model plot (Figure 39). This is because the macro-model assumes that the reflective characteristics of the material behave in the same way in both the visible and infrared wavelengths. This assumption is not correct and will be addressed in the future study of the "as-built" micro-models. Finally, be aware that the same problems with the different yaw steering boundaries and the absence of tunes parameters discussed for the albedo also apply for the IR force.

\section{Spacecraft Radiation}

The thermal imbalance acceleration on the T/P spacecraft is the most difficult of the nonconservative forces to model. However, using the algorithms defined previously, the macro-model approximates the micro-model acceleration profiles very well as shown in Figures 43-51. The micro-model plots were generated with data spaced at 8 degrees in $B^{\prime}$ and 5 degrees in orbit angle. The overall signature of this force is similar to the solar radiation pressure, which is expected since both forces are primarily driven by the spacecraft-Sun orientation. However, Earth albedo and IR have some effect, especially at low B' where they have the greatest magnitude, as demonstrated in temperature plots (Figures 52-67).

The large residuals between the micro and macro-models in the along-track and radial directions (Figures 49 and 51) at low B' are attributed to the fact that the thermal imbalance macro-model does not consider heating due to Earth albedo or IR. This signal dies off quickly as the spacecraft moves into sinusoidal yaw where the solar array has less projected area in the planet direction and the X-face is oriented toward the sun at all times. Also, the discontinuities seen in the along-track macro-model (Figure 44) at this yaw steering boundary are due to instantaneous changes in the macro-model plate shadow boundary location. This is most pronounced for the $X$ - face in the $0^{\circ}$ to $90^{\circ}$ orbit angle region where the plate goes from shadow to maximum illumination as soon as the spacecraft crosses $B^{\prime}=15^{\circ}$. This same discontinuity effect, coupled with differences in the 
shadow boundary location discussed previously, acts on the $Z+$ plate to create the large spike near $B^{\prime}=56^{\circ}$ in the radial residual plot.

As a further check of the model, the predicted temperature profiles for each plate were compared to selected nodes from the micro-model. Recall that the spacecraft radiation acceleration is driven by the temperature gradient across the plates. Reasonable accelerations can be obtained with unreasonable absolute temperatures. By looking at the temperature behavior emulated by the tailored model, the physical reasonableness of the resulting model is assessed. These comparisons are shown in the temperature profiles shown in Figures 52-67. Note that the micro-model temperature profiles are from select nodes on the spacecraft and may not be representative of what is happening on the entire plate. Figures 52-55 demonstrate the macro-model's excellent ability to predict the solar array temperature in all regimes. This is attributed to the fact that the solar array's material properties are nearly homogeneous, it has a low thermal inertia, and it is oriented for maximum solar exposure. The $X+$ face is shown in Figure 56 and 57. Notice that it experiences wild fluctuations at $B^{\prime}=0^{\circ}$ but quickly reaches an almost constant cold equilibrium value as the spacecraft enters sinusoidal yaw and the plate is no longer illuminated. The warming due to Earth IR can be seen between approximately $235^{\circ}$ and $325^{\circ} \Omega$ at low B' on the micro-model plot only (Figure 56). This is also seen in the Z- plots (Figures 66-67). As discussed previously, this effect is not duplicated in the macro-model. Figures 58 and 59 show the $X$ - temperature profile which exhibits the same large temperature fluctuations at low B' as experienced by the $X+$ face. Note the significant change in the profile as soon as the spacecraft begins sinusoidal yaw steering $\left(B^{\prime}=15^{\circ}\right)$. This has a pronounced effect on the thermal imbalance acceleration as outlined previously. Yaw steering causes the dip centered at $\Omega=90^{\circ}$ while Earth occultation causes the larger temperature drop between $\Omega=235^{\circ}$ and $\Omega=325^{\circ}$. Both of these effects disappear as $B^{\prime}$ increases above $56^{\circ}$ where the satellite experiences continuous sunlight and has very little yaw motion. The $\mathrm{Y}+$ temperature profile shows significant deviation between the micro and macro-models. The macro-model values make physical sense when the plate's solar illumination is considered. The temperature consistency shown in the micro-model suggests this particular node is not representative of the $\mathrm{Y}+$ plate and that it might be located in or near the spacecraft interior, where the temperature is known to be nearly constant. The $Y$ - plate also demonstrates difference between the two models, but to a lesser degree. This face, located on the same side as the solar array, experiences illumination and, therefore, heating from rays reflected from the solar array. This interaction is neglected in the macro-model. The $\mathrm{Z}+$ face (Figures 64-65) best demonstrate the albedo effect on the temperature signal in the $\Omega=0^{\circ}$ to $180^{\circ}$ range. The other half of the orbit shows the rapid heating and cooling associated with the plate illumination twice per orbit. Overall, the macro-model performs more than adequately in both acceleration and temperature space. Further verification will be 
possible on-orbit where direct comparisons with telemetered temperature data can be performed.

\section{Modelability and Error Analysis}

The results presented in the previous sections have given a measure of the macro-model success in modeling satellite accelerations. Mission requirements, however, require that model performance be evaluated in terms of radial orbit error. In order to quantify the radial orbit error produced by macro model errors, the following analysis was performed.

The micro-model acceleration histories are considered by default to be "truth". Certainly, they are the result of a very rigorous analysis and are the best representation of "truth" that is currently available. Thus, any mismodeling by the macro-model is represented by the macro-micro model acceleration residual histories previously discussed. During this analysis, each of the macro model force errors were considered individually. This was necessary, since each force was individually tuned to the micro-model. The exception is the Earth albedo and IR, which simply use the solar model tuned parameter values found in Table 5.

GEODYN was modified to include a routine that does a bilinear interpolation (over B' and $\Omega$ ) on the micro-model acceleration data for each of the forces considered (solar, albedo, IR, thermal). Thus, micro-model accelerations were computed at integration step time within the GEODYN software. Figures 68 to 79 show the bilinearly interpolated micro model acceleration histories for a B' region of 10.5 to 36 degrees. Comparisons of these figures with the micro-model acceleration histories discussed in previous sections show that the bilinear interpolation is adequate. The "micro-model GEODYN" version produced four data sets containing true-of-date $X, Y, Z$ orbit data for a 10 day $T / P$ arc, using each of the micro-model accelerations in turn. A 30 second integration step size was used for all runs, and the data generation interval was 180 seconds. The particular 10 day arc spans a B' region of 10.5 to 36 degrees. Therefore, this arc covers the fixed yaw to sinusoidal yaw regime, and the analysis is weighted towards the acceleration residuals in this region. Four separate data reduction runs using the macro-model accelerations were made on the four micro-model generated data sets. Thus, for each individual force considered, the only difference in the force modeling between the data generation and data reduction runs is the particular macro-micro model difference. Only the state, a single drag coefficient, and a single solar radiation pressure coefficient were adjusted over the 10-day arc interval. The adjustment of specific macro-model parameters were not part of this study, and will be addressed extensively in a companion report in preparation. The radial RMS orbit error was computed from the residuals of the macro- 
model-fit orbit to the micro-model-generated orbit.

Table 7 gives the modelability and error analysis summary. The RMS radial orbit errors over a 10 day $T / P$ arc for each of the individual macro-model forces is less then $6 \mathrm{~cm}$, demonstrating that each individual macro-model meets mission requirements. It should be stressed that these results were achieved without adjusting T/P macro-model specific parameters, and that the inclusion of these parameters should improve modelability and reduce the orbit errors.

Some comments about the results in Table 7 are given below. The modelability of the along-track and cross-track albedo and IR accelerations is poor. However, the surface properties used to describe the albedo and IR models were never tuned to the micromodels, and currently the solar model parameter set is employed. The modelability of the accelerations should improve when macro-model parameters are adjusted. Furthermore, the radial component of the albedo and IR accelerations is an order of magnitude larger than the other components. The modelability of this component is quite good. The along-track thermal acceleration also demonstrates deficiencies due to problems in modeling and tuning the $X$ - plate temperature. Further model tuning is necessary for this $X$ - face. The solar radiation pressure is currently modeled at the $10 \%$ level and is anticipated to improve with the tuning of the solar array diffuse and specular reflectivity parameters. 
Table 7. Modelabllity and Error Analysis Summary

\begin{tabular}{|c|c|c|c|}
\hline FORCE & $\begin{array}{l}\text { RMS of Micro } \\
\text { Model Force for } \\
B^{\prime} \text { of } 0^{\circ} \text { to } 88^{\circ} \\
\text { and Orbit Angle } \\
\text { of } 0^{\circ} \text { to } 360^{\circ}\end{array}$ & $\begin{array}{l}\text { RMS of Macro- } \\
\text { Micro Model } \\
\text { Residuals }\end{array}$ & $\begin{array}{l}\text { RMS Radial } 10 \\
\text { Day Orbit Error } \\
\text { (For All } \\
\text { Components) }\end{array}$ \\
\hline $\begin{array}{l}\text { Solar Along-Track } \\
\text { Solar Cross-Track } \\
\text { Solar Radial }\end{array}$ & $\begin{array}{l}3.2 \times 10^{-8} \mathrm{M} / \mathrm{S}^{2} \\
4.5 \times 10^{-8} \mathrm{M} / \mathrm{S}^{2} \\
2.7 \times 10^{-8} \mathrm{M} / \mathrm{S}^{2} \\
\end{array}$ & $\begin{array}{l}4.3 \times 10^{-9} \mathrm{M} / \mathrm{S}^{2} \\
4.3 \times 10^{-9} \mathrm{M} / \mathrm{S}^{2} \\
4.9 \times 10^{-0} \mathrm{M} / \mathrm{S}^{2}\end{array}$ & \\
\hline \multicolumn{3}{|c|}{ Total Radial Orbit Error for 10 Day Arc $\rightarrow$} & $5.1 \mathrm{~cm}$ \\
\hline $\begin{array}{l}\text { Albedo Along-Track } \\
\text { Albedo Cross-Track } \\
\text { Albedo Radial }\end{array}$ & $\begin{array}{l}6.1 \times 10^{-10} \mathrm{M} / \mathrm{S}^{2} \\
8.3 \times 10^{-10} \mathrm{M} / \mathrm{S}^{2} \\
4.6 \times 10^{-9} \mathrm{M} / \mathrm{S}^{2} \\
\end{array}$ & $\begin{array}{l}2.3 \times 10^{-10} \mathrm{M} / \mathrm{S}^{2} \\
3.3 \times 10^{-10} \mathrm{M} / \mathrm{S}^{2} \\
2.4 \times 10^{-10} \mathrm{M} / \mathrm{S}^{2}\end{array}$ & \\
\hline \multicolumn{3}{|c|}{ Total Radial Orbit Error for 10 Day Arc $\rightarrow$} & $2.1 \mathrm{~cm}$ \\
\hline $\begin{array}{l}\text { IR Along-Track } \\
\text { IR Cross-Track } \\
\text { IR Radial }\end{array}$ & $\begin{array}{l}6.1 \times 10^{-10} \mathrm{M} / \mathrm{S}^{2} \\
7.4 \times 10^{-10} \mathrm{M} / \mathrm{S}^{2} \\
5.6 \times 10^{-9} \mathrm{M} / \mathrm{S}^{2} \\
\end{array}$ & $\begin{array}{l}5.4 \times 10^{-10} \mathrm{M} / \mathrm{S}^{2} \\
5.3 \times 10^{-10} \mathrm{M} / \mathrm{S}^{2} \\
5.4 \times 10^{-10} \mathrm{M} / \mathrm{S}^{2}\end{array}$ & \\
\hline \multicolumn{3}{|c|}{ Total Radial Orbit Error for 10 Day Arc $\rightarrow$} & $2.2 \mathrm{~cm}$ \\
\hline $\begin{array}{l}\text { Thermal Along-Track } \\
\text { Thermal Cross-Track } \\
\text { Thermal Radial }\end{array}$ & $\begin{array}{l}2.0 \times 10^{-9} \mathrm{M} / \mathrm{S}^{2} \\
3.6 \times 10^{-9} \mathrm{M} / \mathrm{S}^{2} \\
1.6 \times 10^{-9} \mathrm{M} / \mathrm{S}^{2} \\
\end{array}$ & $\begin{array}{l}1.0 \times 10^{-9} \mathrm{M} / \mathrm{S}^{2} \\
5.9 \times 10^{-10} \mathrm{M} / \mathrm{S}^{2} \\
5.5 \times 10^{-10} \mathrm{M} / \mathrm{S}^{2}\end{array}$ & \\
\hline \multicolumn{3}{|c|}{ Total Radial Orbit Error for 10 Day Arc $\rightarrow$} & $5.2 \mathrm{~cm}$ \\
\hline
\end{tabular}

The above analysis shows that with no adjustment of the macro-model surface-specific parameters and a minimal adjusted parameter set, the individual macro-model errors meet mission requirements. However, the error produced by the sum of the nonconservative forces must meet the $6 \mathrm{~cm}$ radial RMS mission requirement. This analysis could not be properly performed since a combined force parameter set has not yet been developed but will be addressed in a companion paper. In order to get an estimate of the combined force error, the above analysis was performed using the combined micro and macro-model forces. The thermal model tuned parameter set was used in this analysis. Adjusting the state, a single drag coefficient, and a single solar radiation pressure coefficient the radial RMS orbit error of a 10 day arc was $6.98 \mathrm{~cm}$. A short run was made including the adjustment of the solar array front diffuse reflectivity 
parameter and demonstrated marked improvement, giving a flavor for the type of improvement expected when macro-model parameters are adjusted. 


\section{CONCLUSIONS}

\section{Summany}

In order to meet precision orbit determination requirements for geodetic satellite missions, specifically TOPEX/Poseidon, detailed models of the radiative forces acting on the spacecraft have been constructed. Solar, Earth albedo, Earth infrared, and the spacecraft's thermal radiation effects have all been considered. A detailed finite element analysis has been performed to compute the total force and induced accelerations acting on the satellite. This required a precise description of the satellite shape, material properties, and attitude control algorithm. Because these models are too computationally intensive to be incorporated into the orbit determination software, a more simplistic model which approximates the finite element acceleration profiles has been developed. It is based on depicting the satellite as a combination of flat plates and computing the nonconservative forces acting on each plate independently. These acceleration vectors are summed to produce the overall effect on the satellite center-of-mass. For T/P, a boxwing shape is used. Each plate has associated parameters which can be adjusted to improve model performance with respect to the micro-model analysis and, when the spacecraft is on orbit, to the tracking observations. The adequacy of these macromodels have been assessed through direct comparison with the micro-models. The effect of these residuals on the orbit error budget has been studied. Analyses indicate these precise models individually meet mission requirements. It has been shown that improved modelability will be achieved when macro model specific parameters are adjusted.

\section{Future Work}

With the incorporation of the macro-models into the GEODYN orbit determination code [Putney, 1991], an intensive study of parameter recoverability and correlation can and will be undertaken. Once Fairchild supplies the T/P "as-built" models, all of the micro-models will be reiterated and the box-wing parameters will be tailored to these reiterated solutions. After launch the actual on-orbit satellite performance as sensed from laser tracking and telemetered temperatures will be used to further refine these values.

Additional error analysis with the "as-built" tuned macro-model parameters needs to be performed. This will enable the determination of the proper orbit error due to the sum of the macro-model force errors. Macro-model specific parameters need to be adjusted to improve modelability. Finally, error analysis using simulated laser tracking must be performed. This is because the model performance is to be evaluated in terms of radial 
orbit error in computations supported exclusively by laser tracking.

When adding the macro-models to the GEODYN code, every attempt is being made to make the code as general as possible rather than T/P specific. Any satellite specific code is isolated to facilitate the change to other spacecraft. Therefore, these same algorithms can be used to support improved orbit determination for other satellites. Specifically, two European satellites, SPOT-2 and ERS-1, are well suited to this task as are the satellites forming the United States' GPS constellation.

With improvements in static gravitational and tidal force models, our attention must now focus on other contributing orbit error sources. The days of treating complex geometric satellites as cannonballs in orbit determination are over. The model development described in this paper represents a first step in addressing what may become the most dominant error source in precision orbit determination in the near future. 


\section{REFERENCES}

Kivelson, M. G., The Solar System, Observations and Interpretations, Vol. IV, PrenticeHall, 1986.

Knocke, P. C., Ries, J. C., and B. D. Tapley, "Earth Radiation Pressure Effects on Satellites," Proceedings of the AIAAIAAS

Astrodynamics Conference, August 1988, pp.577-586.

Marshall, J.A, et al., "Modeling Radiation Forces Acting on Satellites for Precision Orbit Determination," AAS-91-357, AAS/AIAA Astrodynamics Conference, August 1991.

Milani, A. et al., Non-Gravitational Perturbations and Satellite Geodesy, Adam Hilger, 1987.

Perrygo, C., "TOPEX Satellite Yaw Maneuvers," Fairchild IOC REF: 968:SE:87-074, 11 November 1987.

Putney, B. H. et al., "GEODYN II System Description," STX Contractor Report, Lanham, MD, 1991.

Rosborough, G.W. and P. Antreasian, "Radiation Force Modeling for the TOPEX/POSEIDON Spacecraft," AIAA-90-2895, AIAAAAS Astrodynamics Conference, August 1990.

Stewart, R., Fu, L. L., and M. Lefebvre, "Science Opportunities from the TOPEX/Poseidon Mission," JPL Pub. 86-18, July 1986.

Wilson, R. C. and H. S. Hudson, "Solar Luminosity Variations in Solar Cycle 21," Nature, Vol. 332, 1988, pp. 810-812.

Zimbelman, D. "Final Version of TOPEX EULERC Subroutine," Fairchild IOC REF: GNC:TOPEX:89-229, 17 October 1989. 


\section{ACKNOWLEDGEMENTS}

The authors wish to thank the TOPEX/Poseidon Project for the important support we have received enabling us to pursue these advanced T/P nonconservative force models for precision orbit determination. 


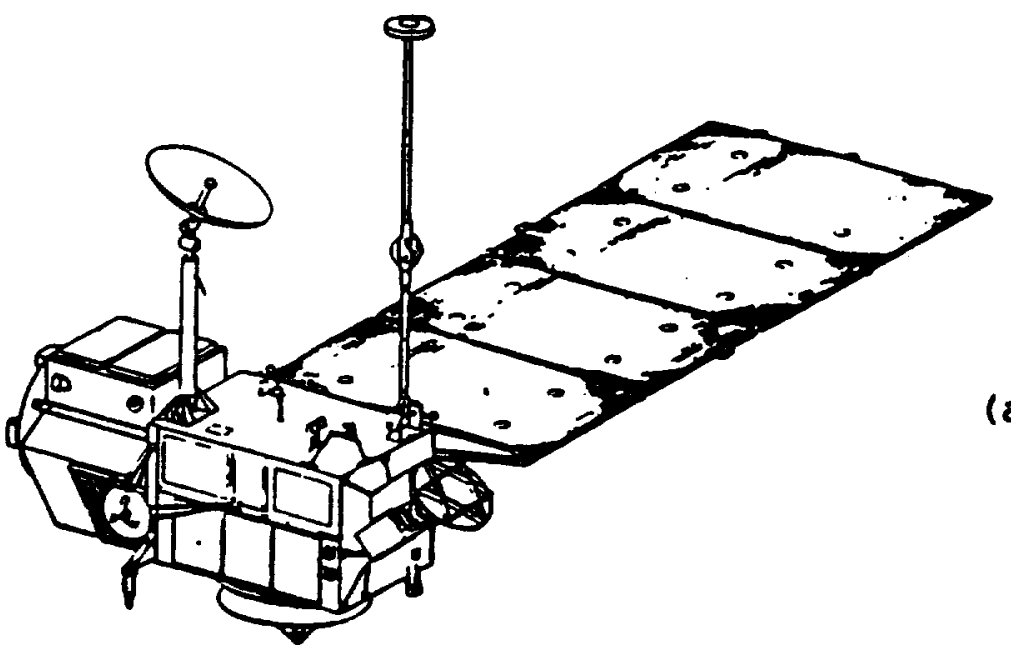

(a)

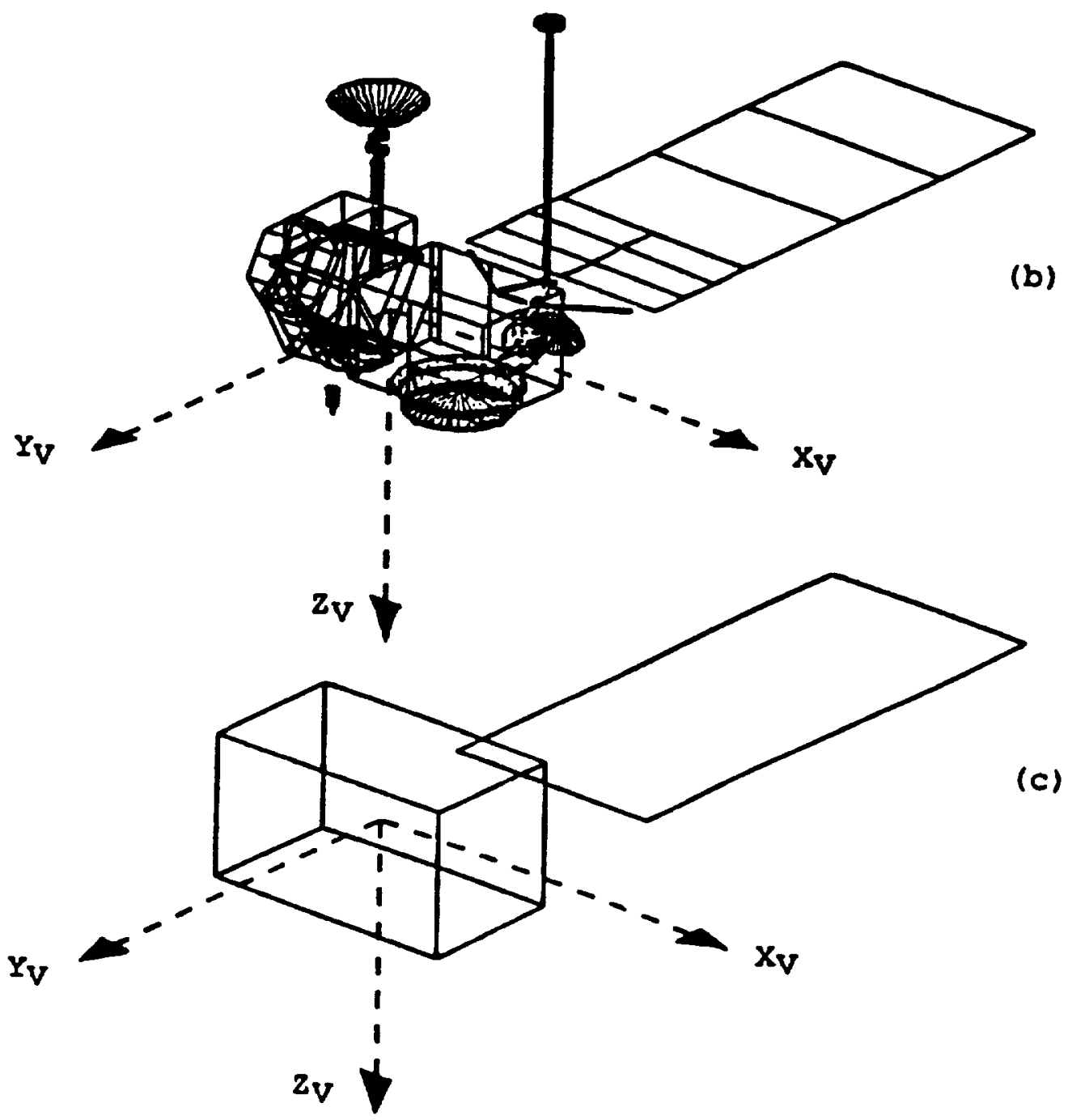

Figure 1. (a) The TOPEX/Poseidon Spacecraft, (b) Micro-Model Approximation, (c) Macro-Model Approximation 


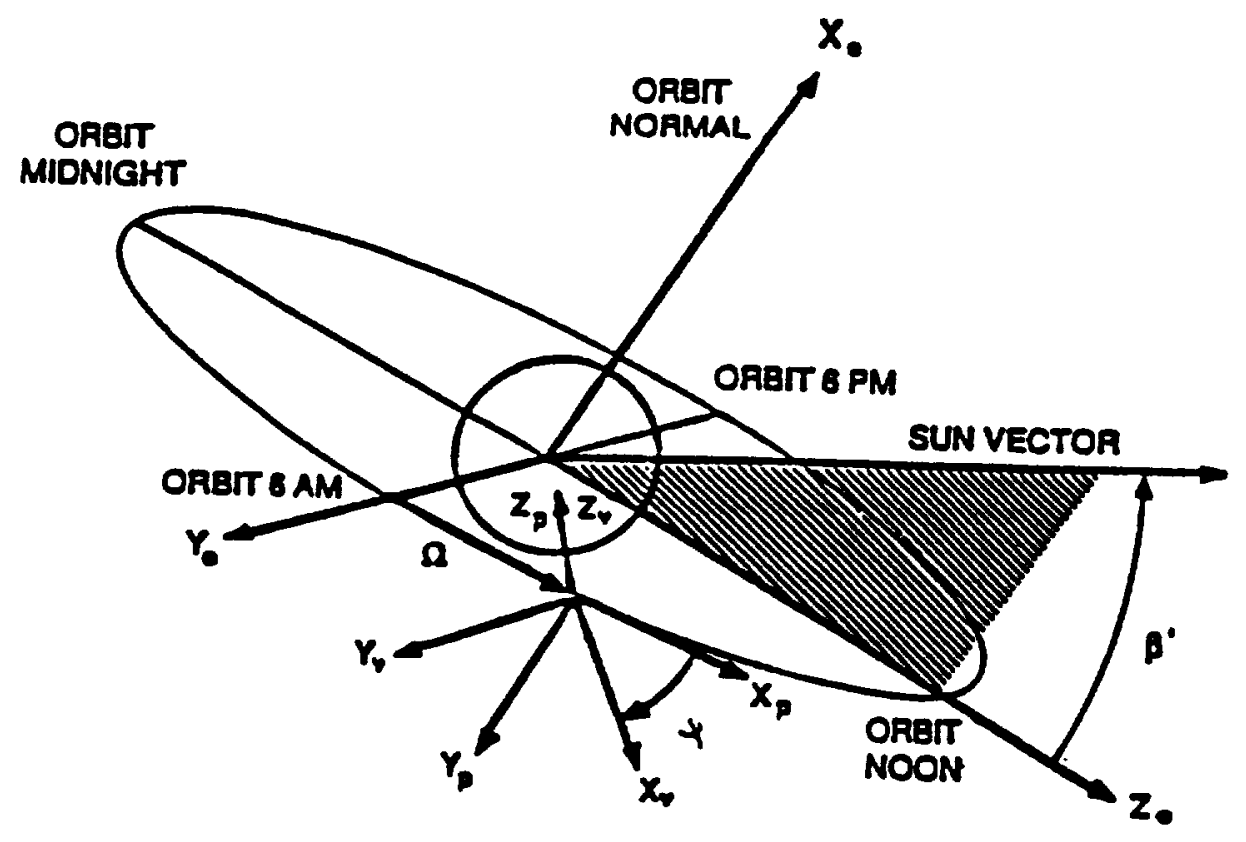

Figure 2. TOPEX/Poseidon Inertial Coordinate System (Perrygo, 1987)

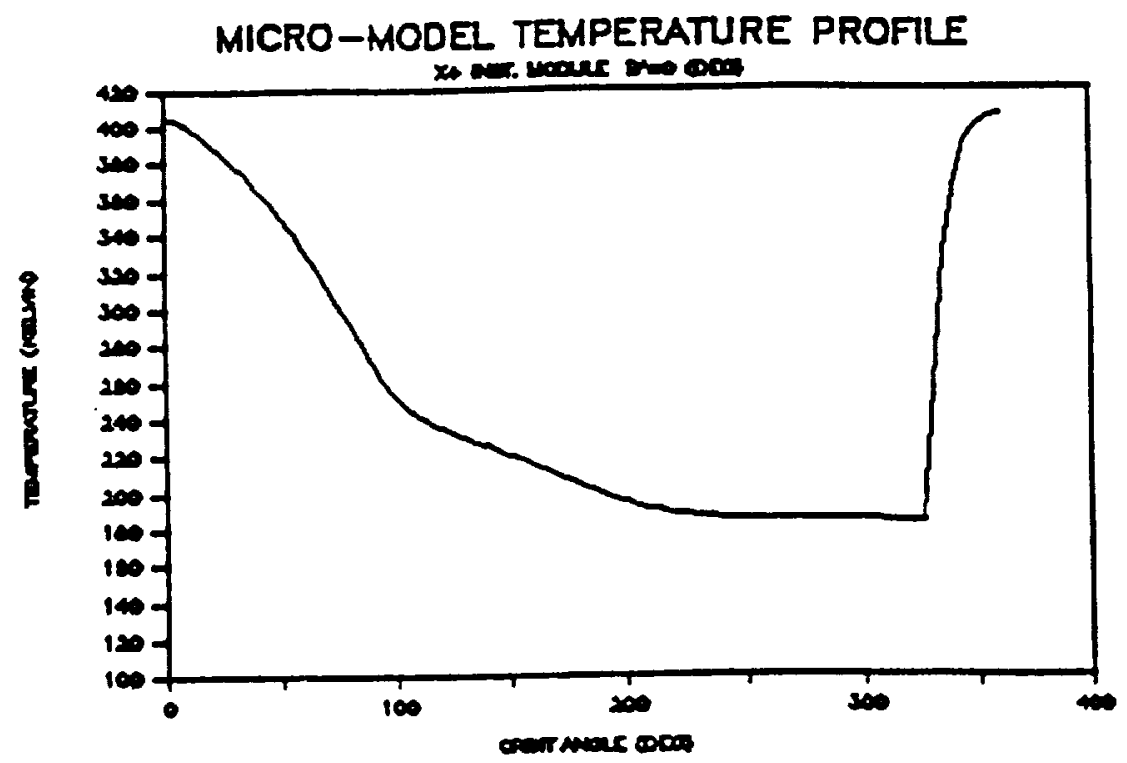

Figure 3. $T / P X+$ Plate Node Temperature Profile Over One Revolution at $B^{\prime}=0^{\circ}$ 


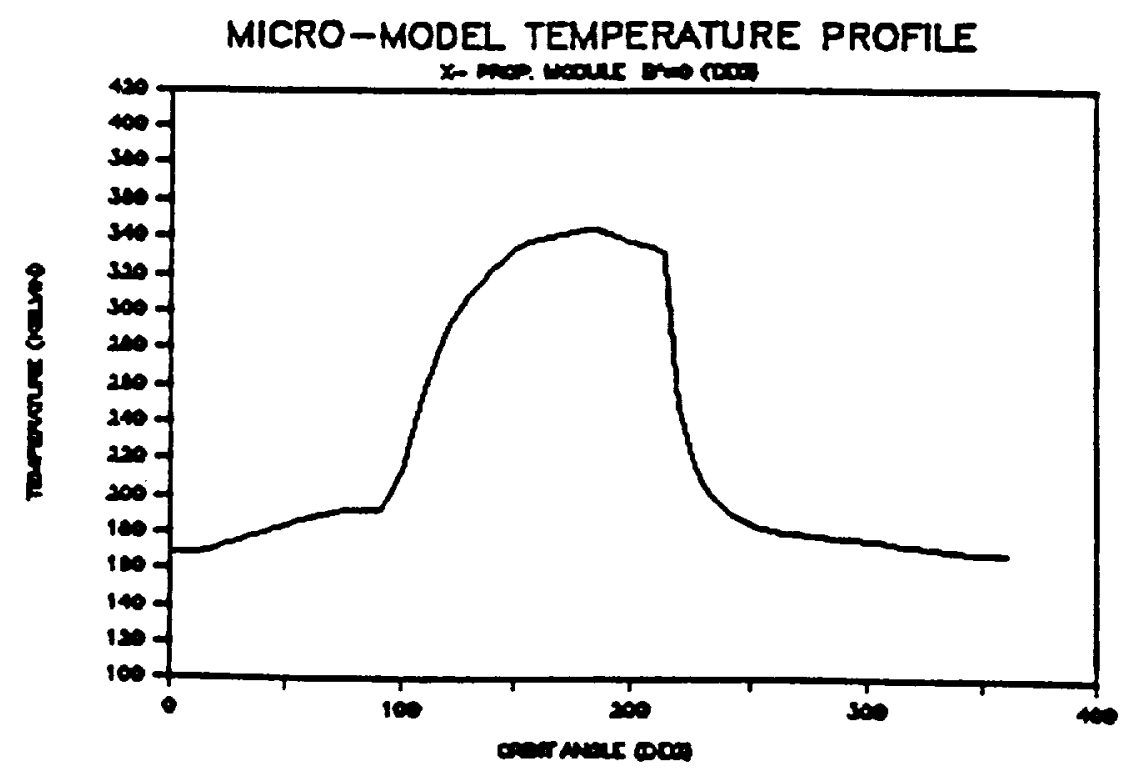

Figure 4. T/O X-Plate Node Temperature Profile Over One Revolution at $B^{\prime}=0^{\circ}$

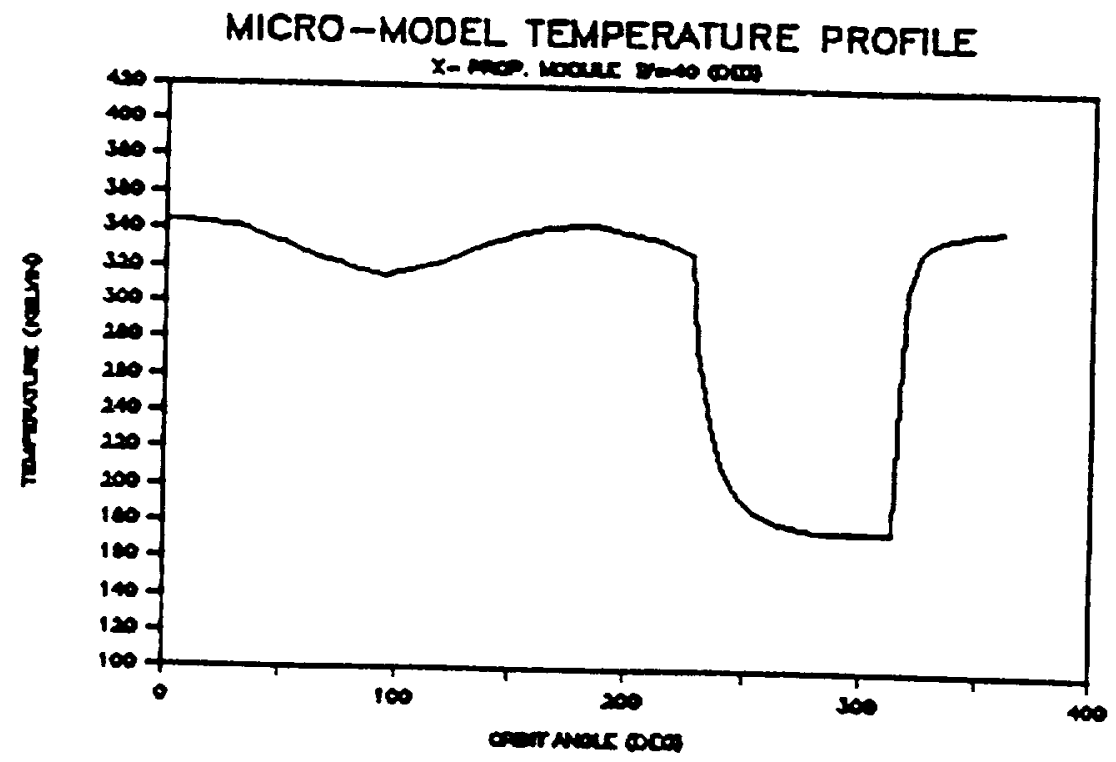

Figure 5. T/P X-Plate Node Temperature Profile Over When Revolution at $B^{\prime}=40^{\circ}$ 


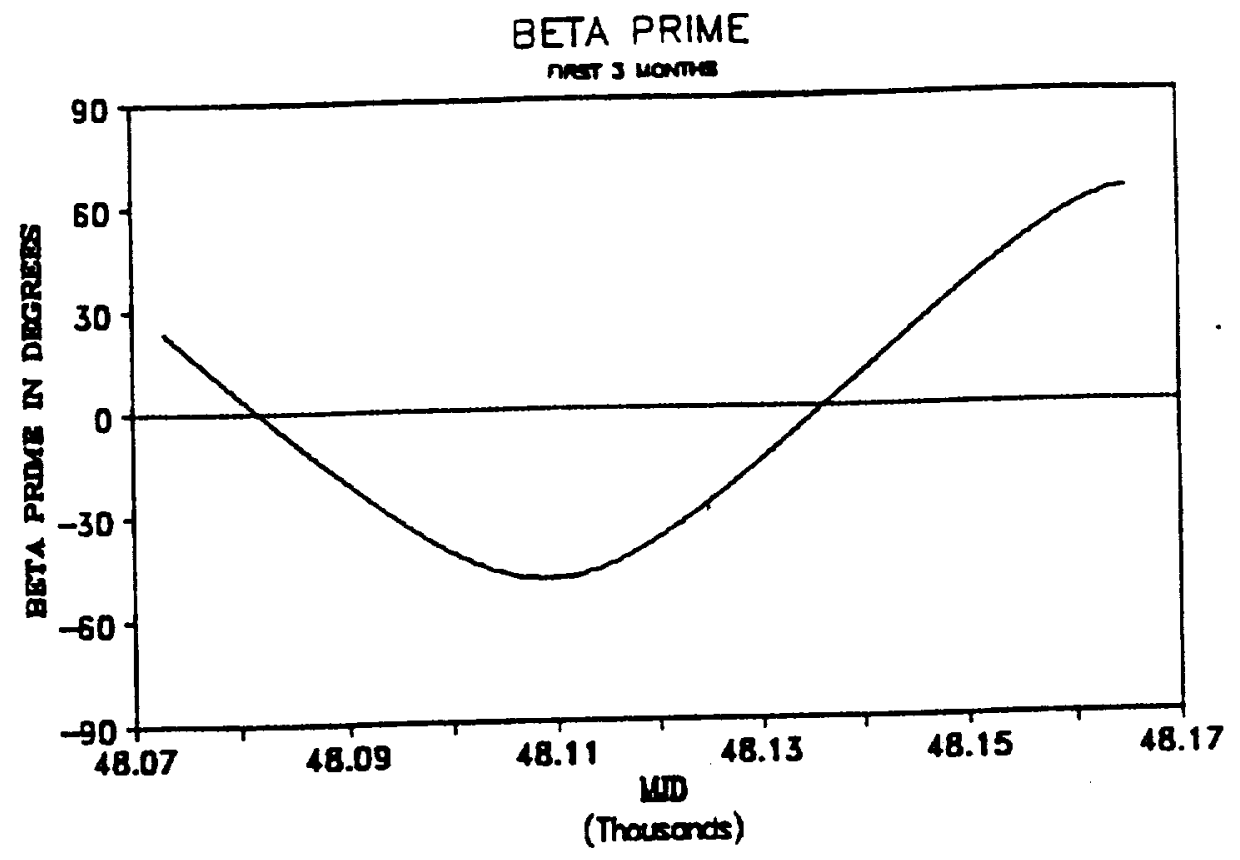

Figure $6(a)$. B' Variation Over the First 3 Months of a 1 Year T/P Orblt

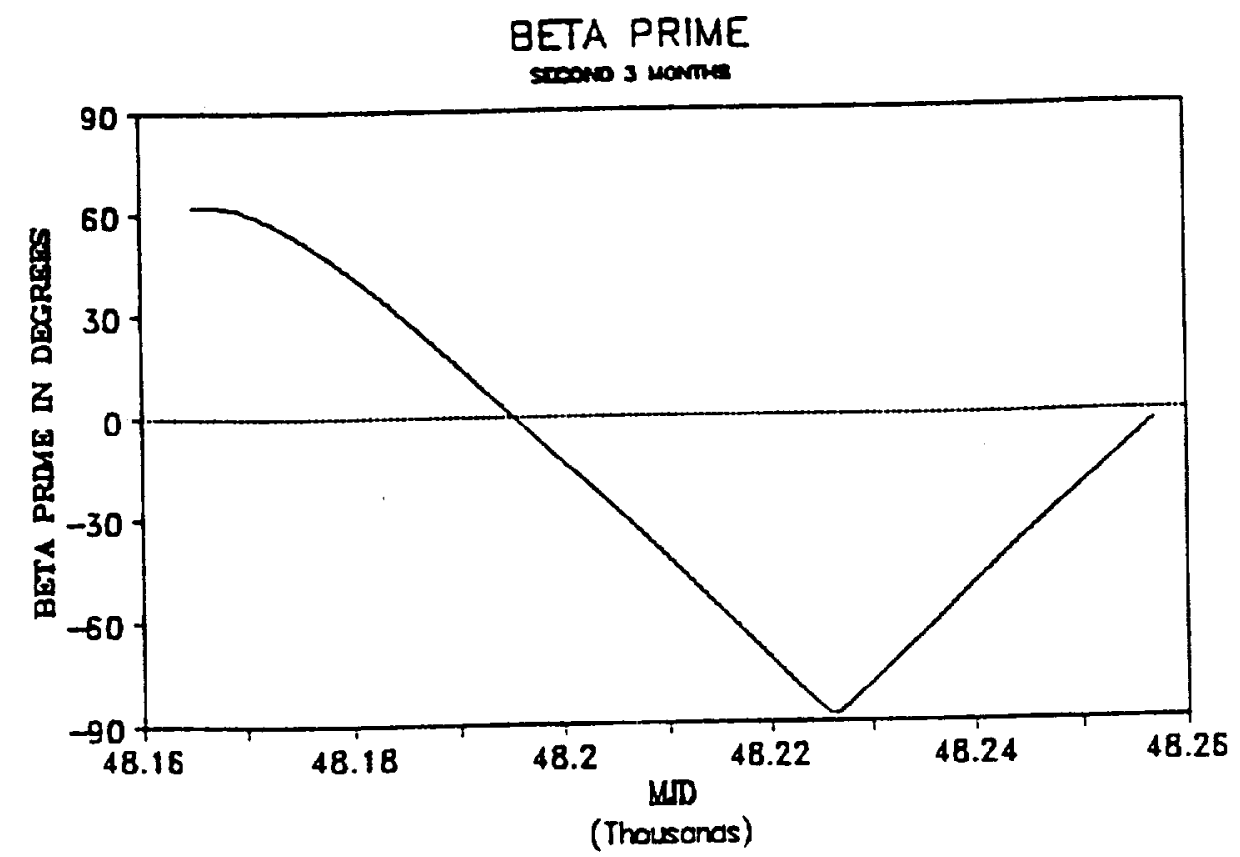

Figure $6(b)$. B' Variation Over the Second 3 Months of a 1 Year $T / P$ Orbit 


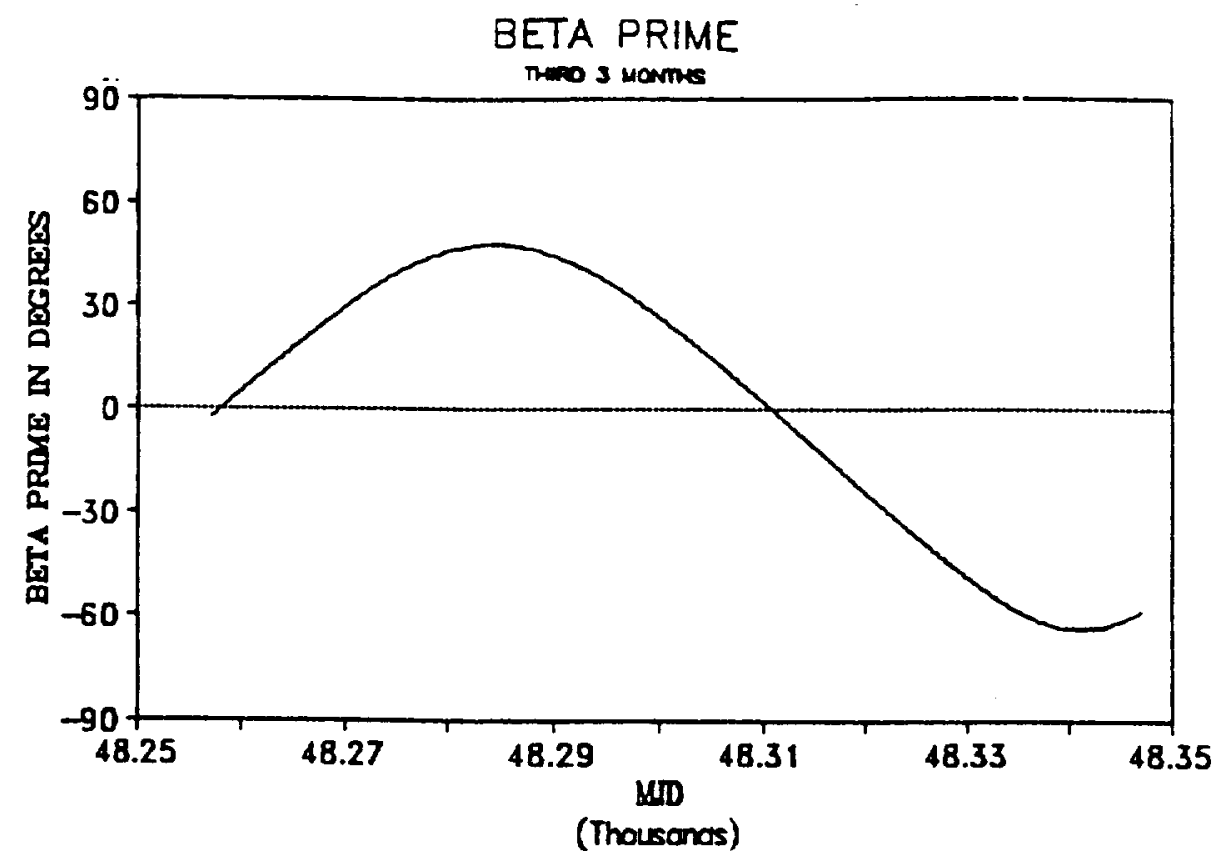

Figure 6(c). B' Variation Over the Third 3 Months of a I Year T/P Orbit

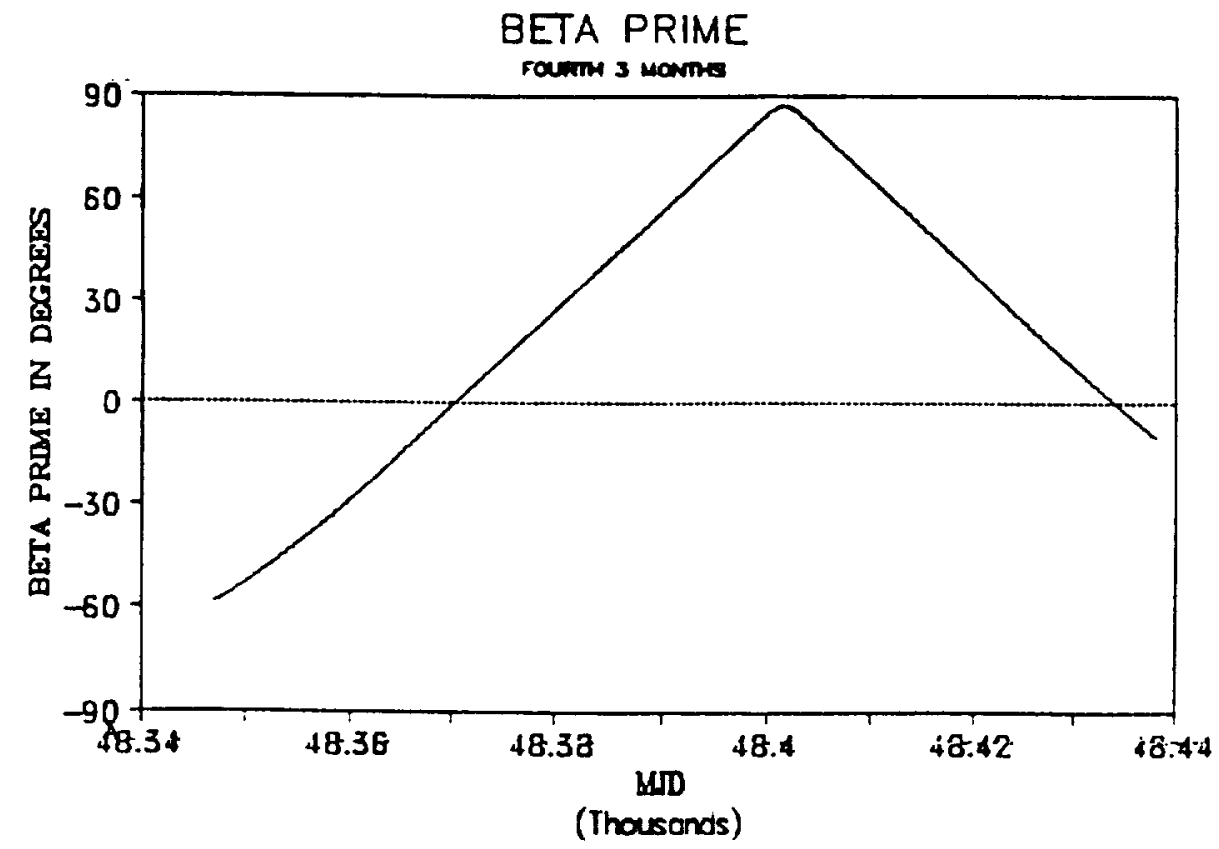

Figure $6(d)$. B' Variation Over the Fourth 3 Months of a I Year T/P Orbit 


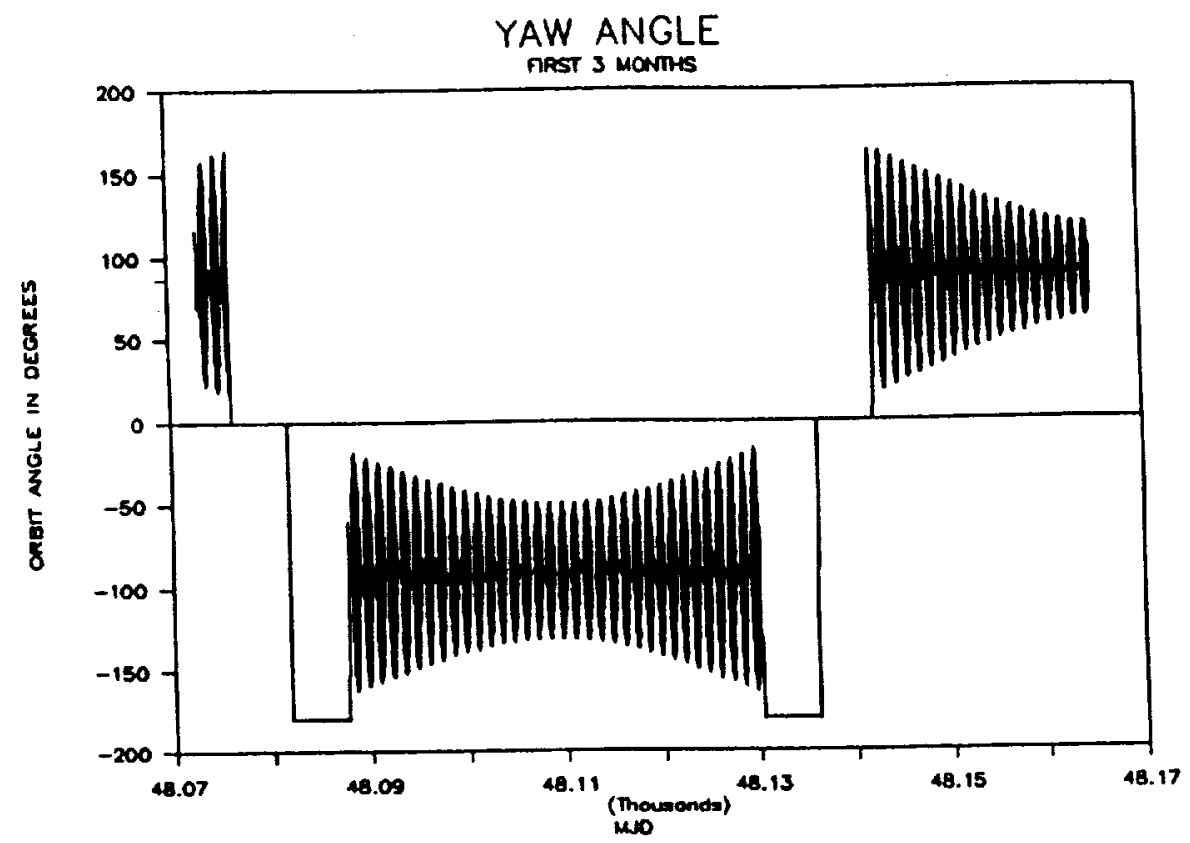

Figure 7(a). First 3 Months of T/P Yaw Angle Variation Over One Year

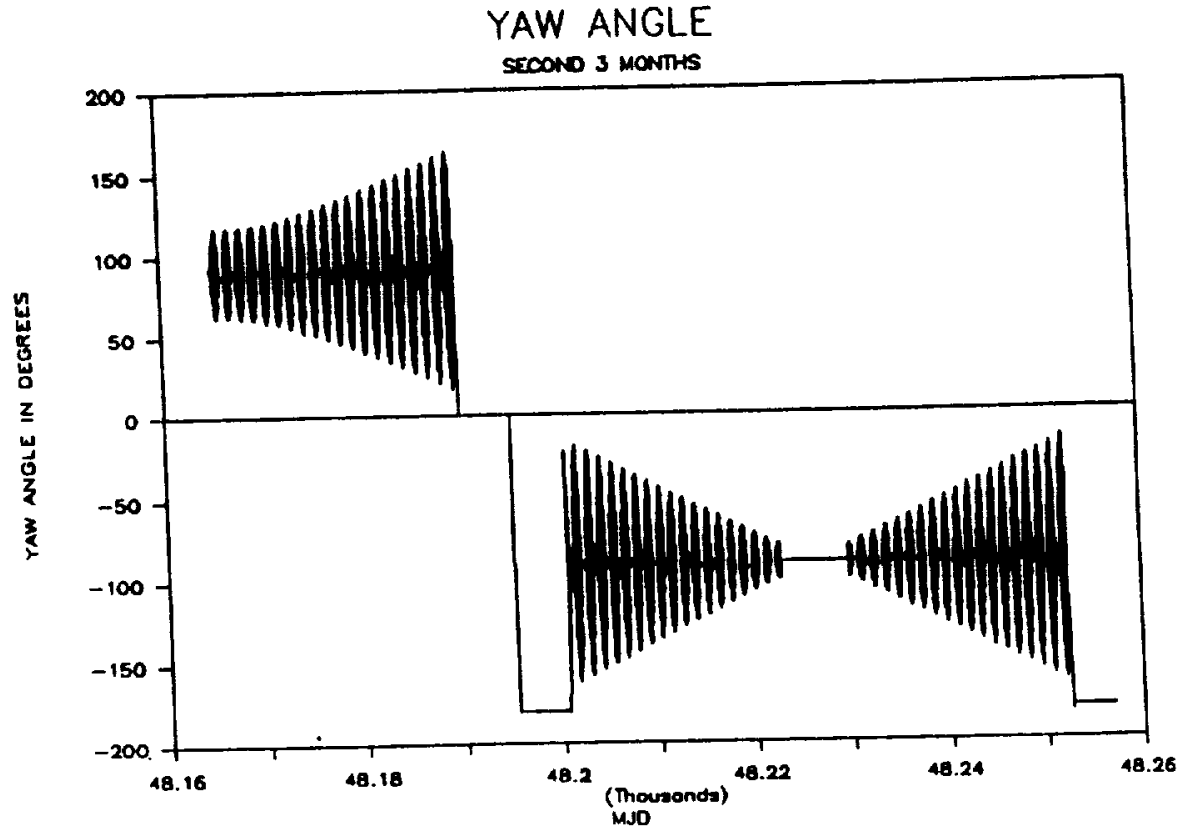

Figure 7(b). Second 3 Months of T/P Yaw Angle Variation Over One Year 


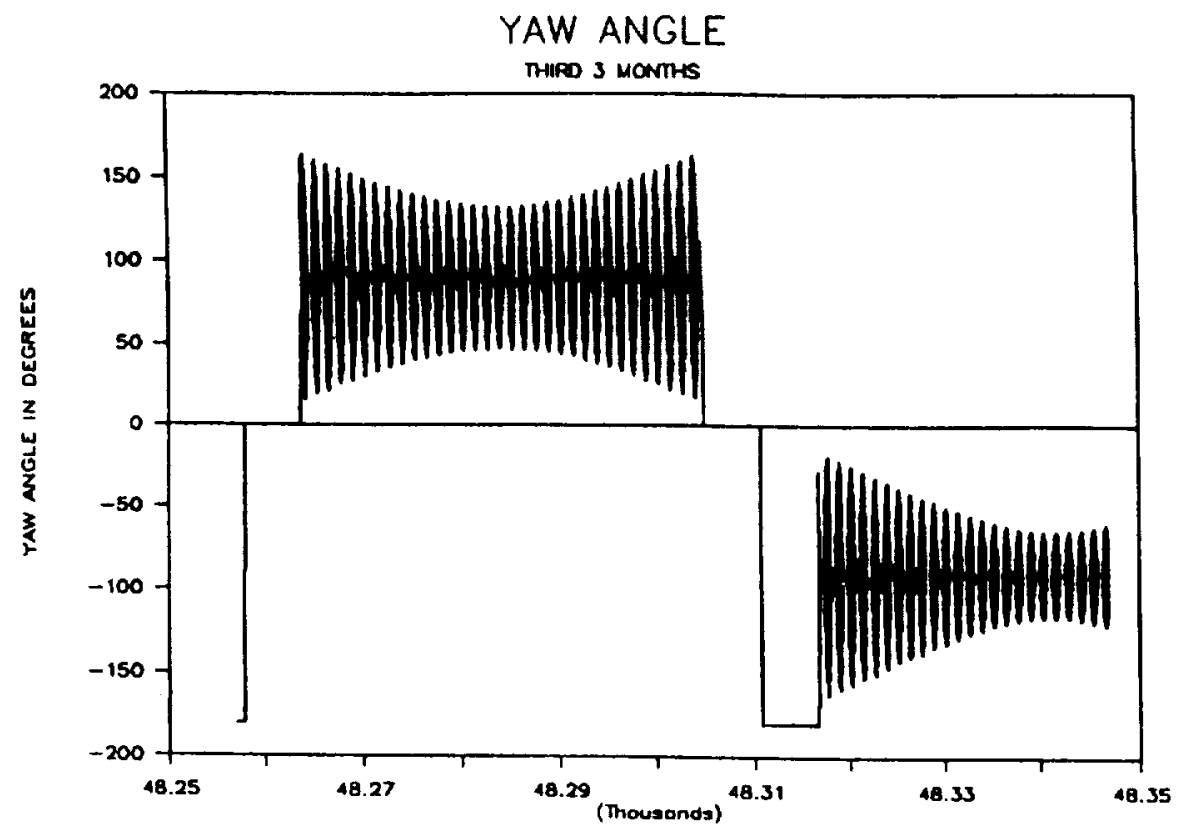

Figure $7(\mathrm{c})$. Third 3 Months of T/P Yaw Angle Variation Over One Year

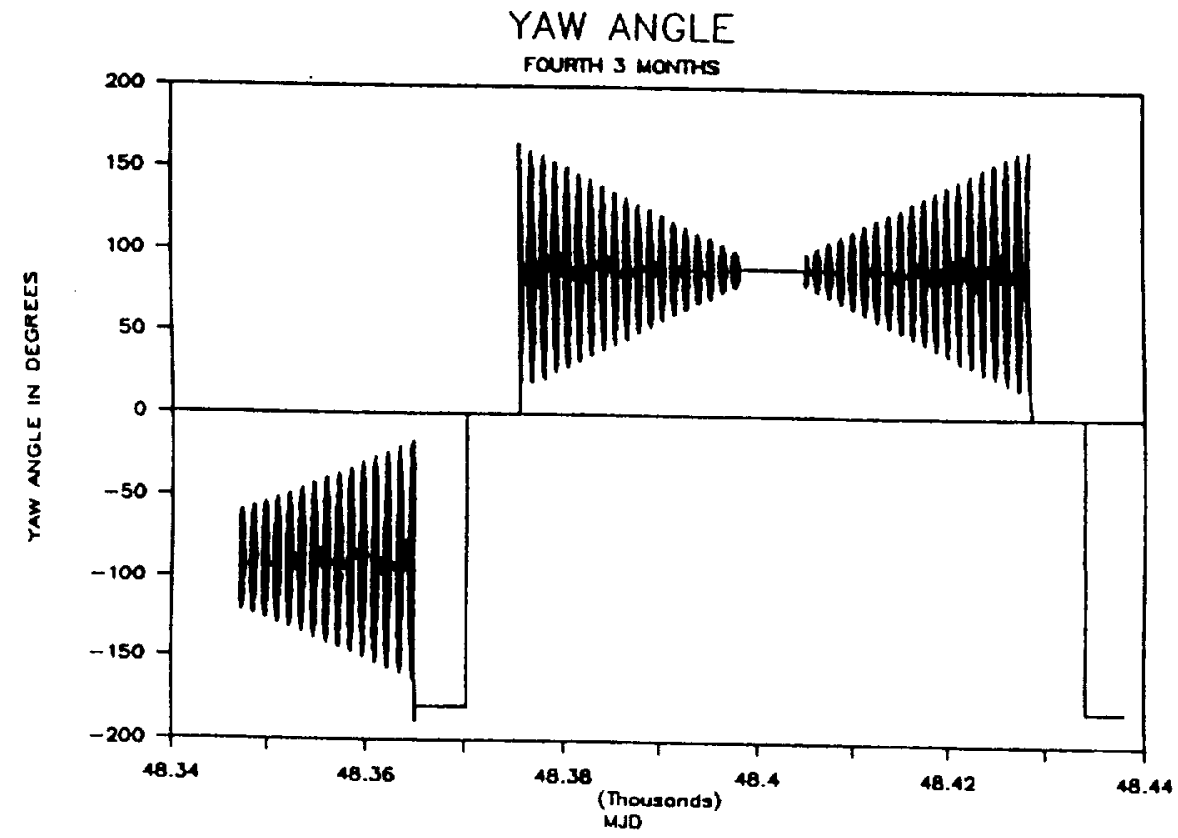

Figure $7(d)$. Fourth 3 Months of T/P Yaw Angle Variation Over One Year 


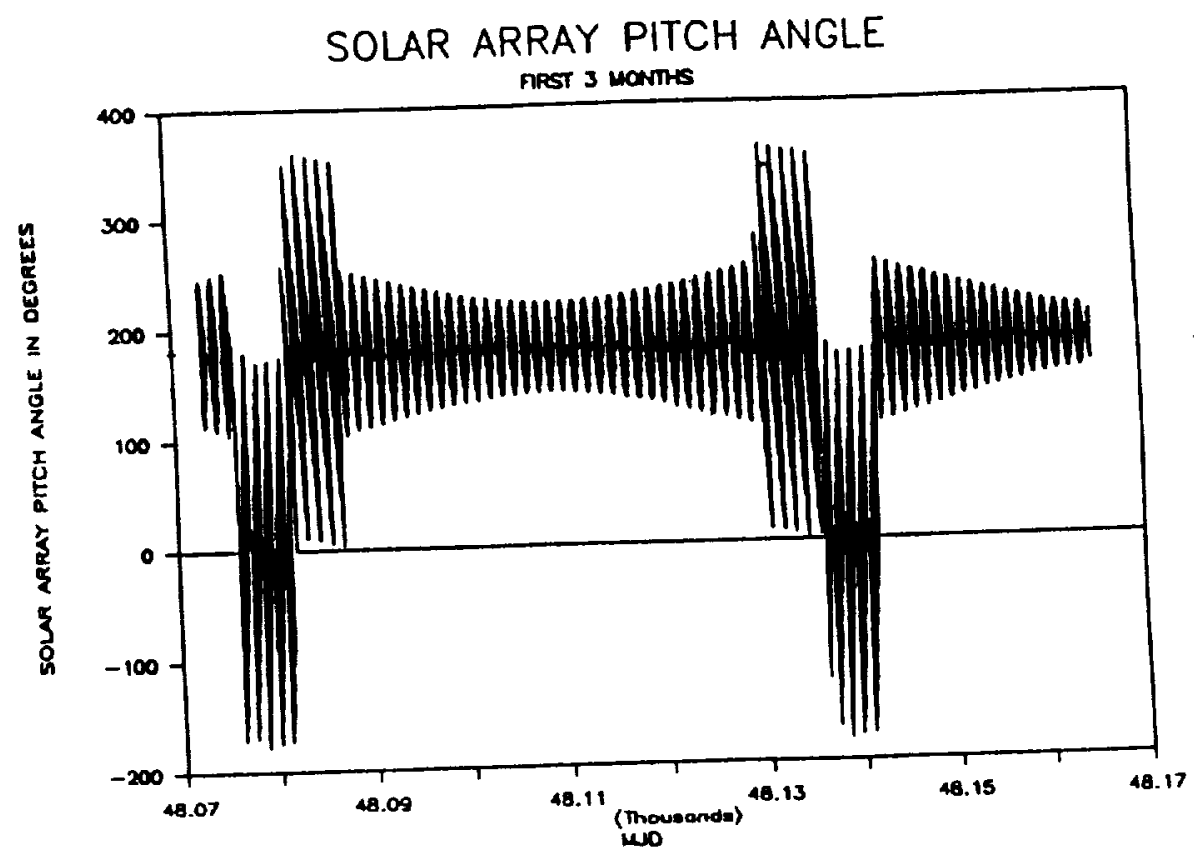

Figure $8(a)$. First 3 Months of T/P Solar Array Pitch Angle Variation Over I Year

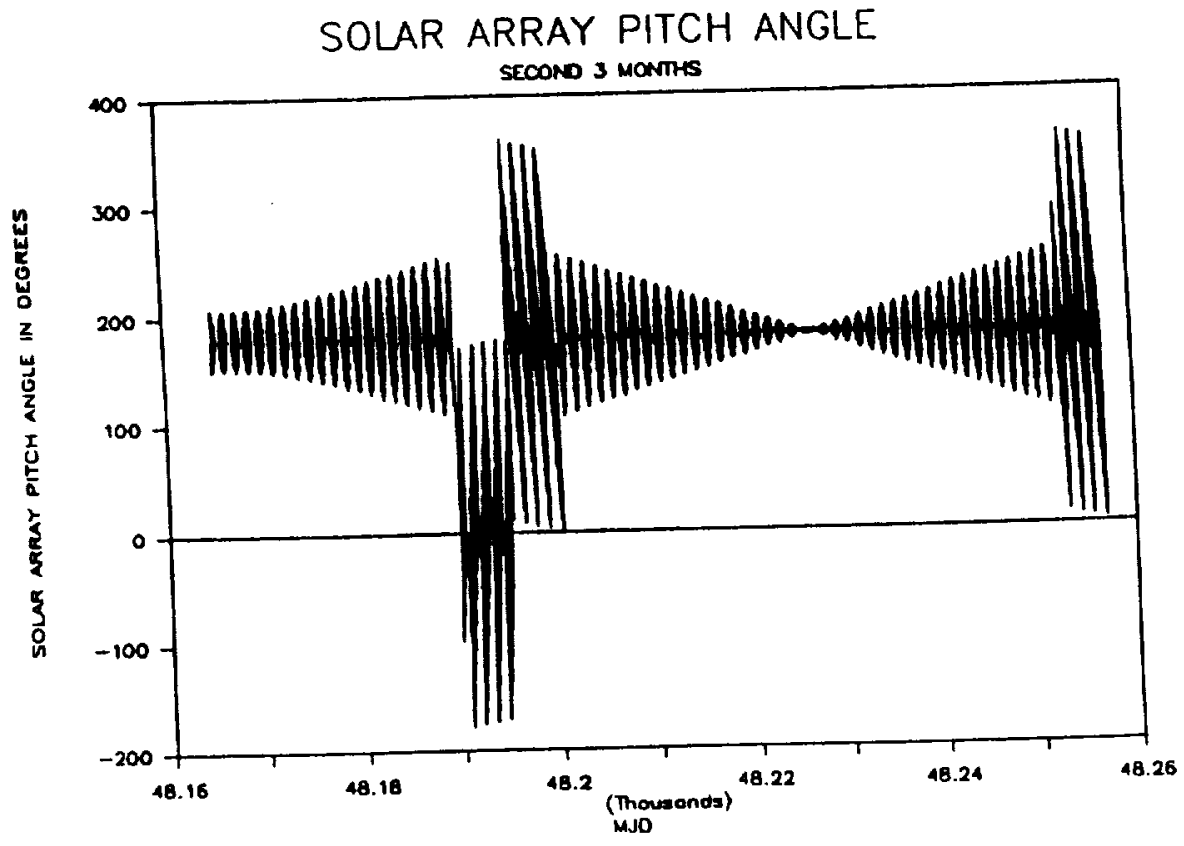

Figure $8(b)$. Second 3 Months of T/P Solar Array Pitch Angle Variation Over 1 Year 


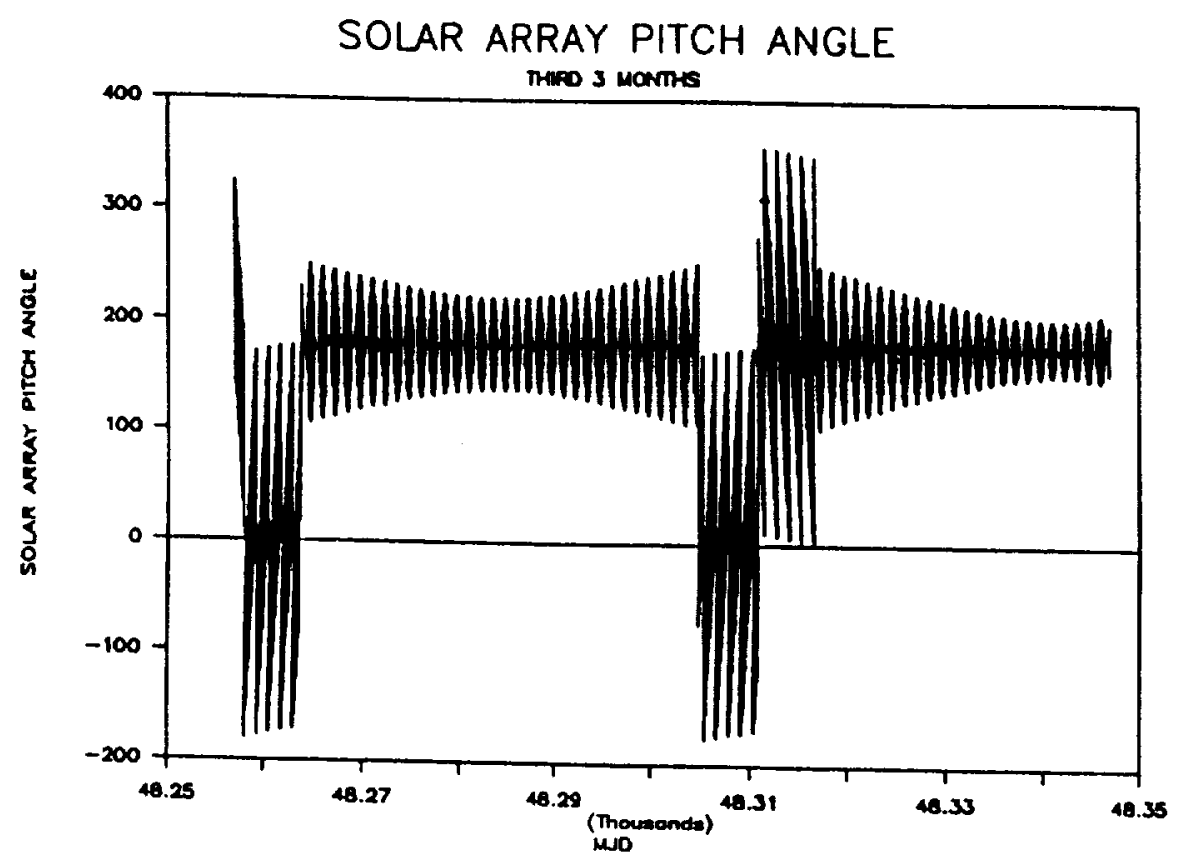

Figure 8(c). Third 3 Months of T/P Solar Array Pitch Angle Variation Over 1 Year

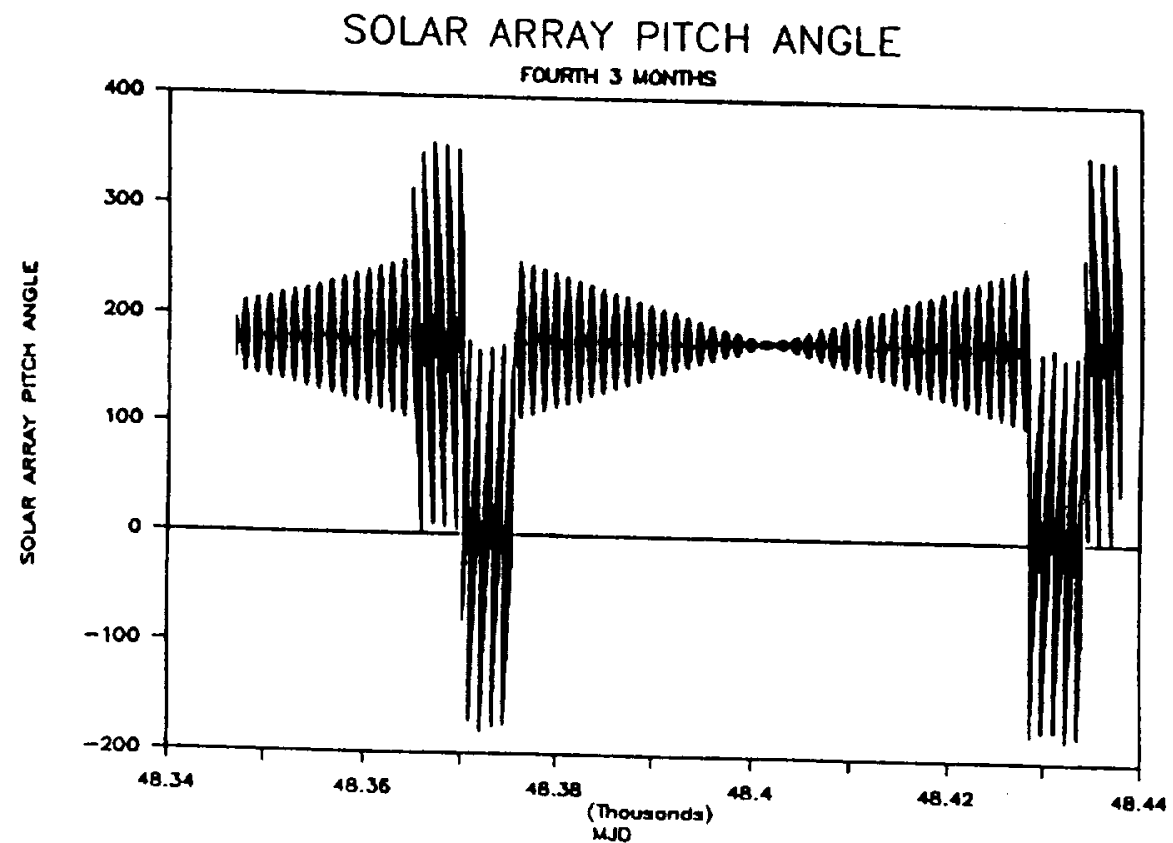

Figure 8(d). Fourth 3 Months of T/P Solar Array Pitch Angle Variation Over 1 Year 


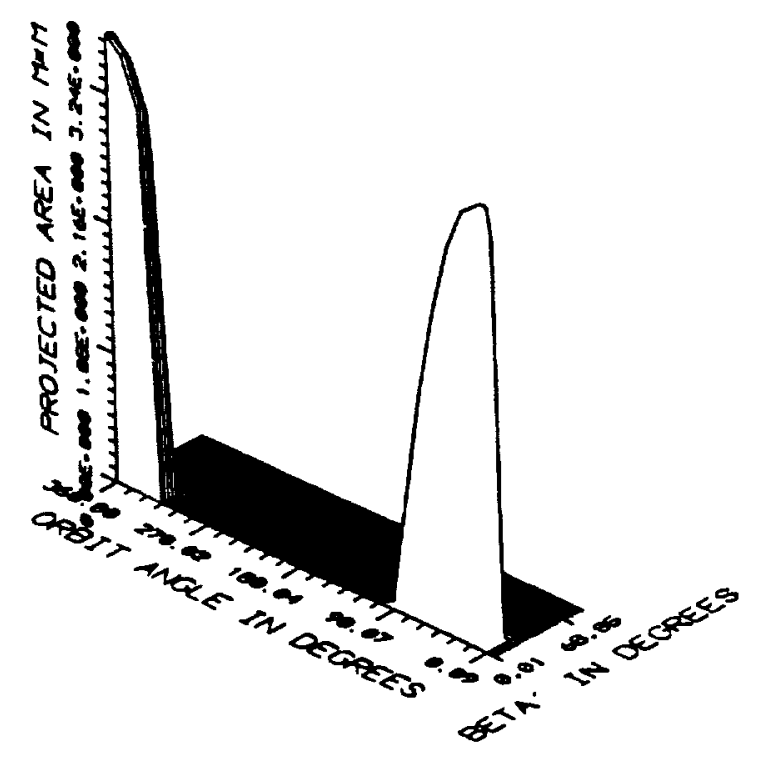

Figure 9. X+ Plate Projected Area in the Sun Dlrection

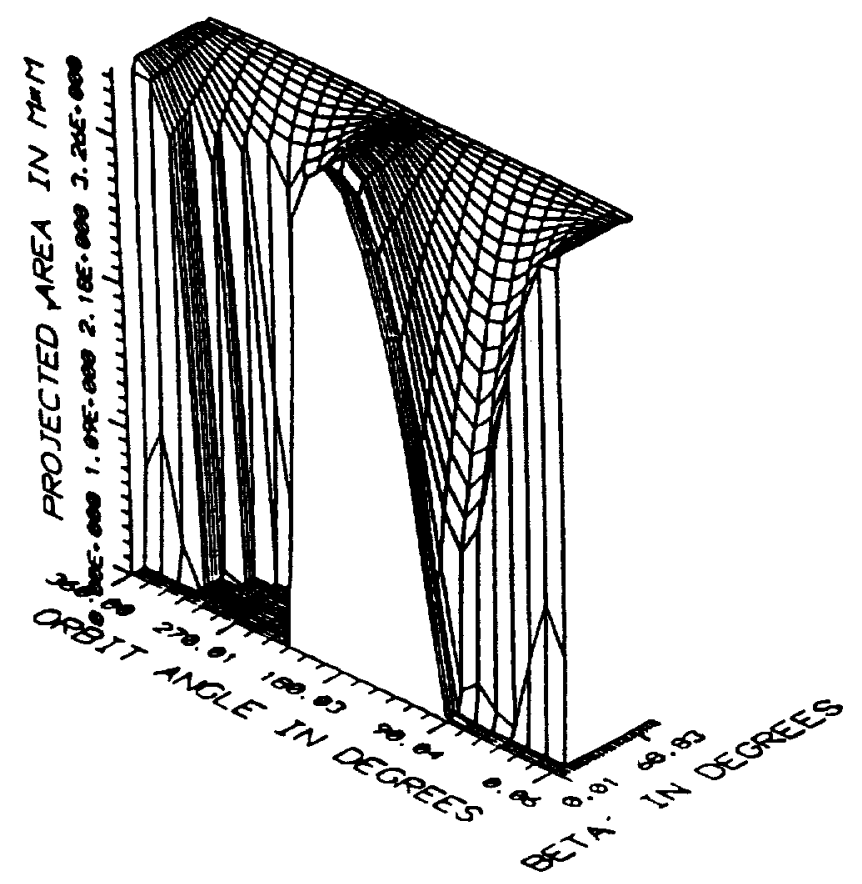

Figure 10. X-Plate Projected Area in the Sun Direction 


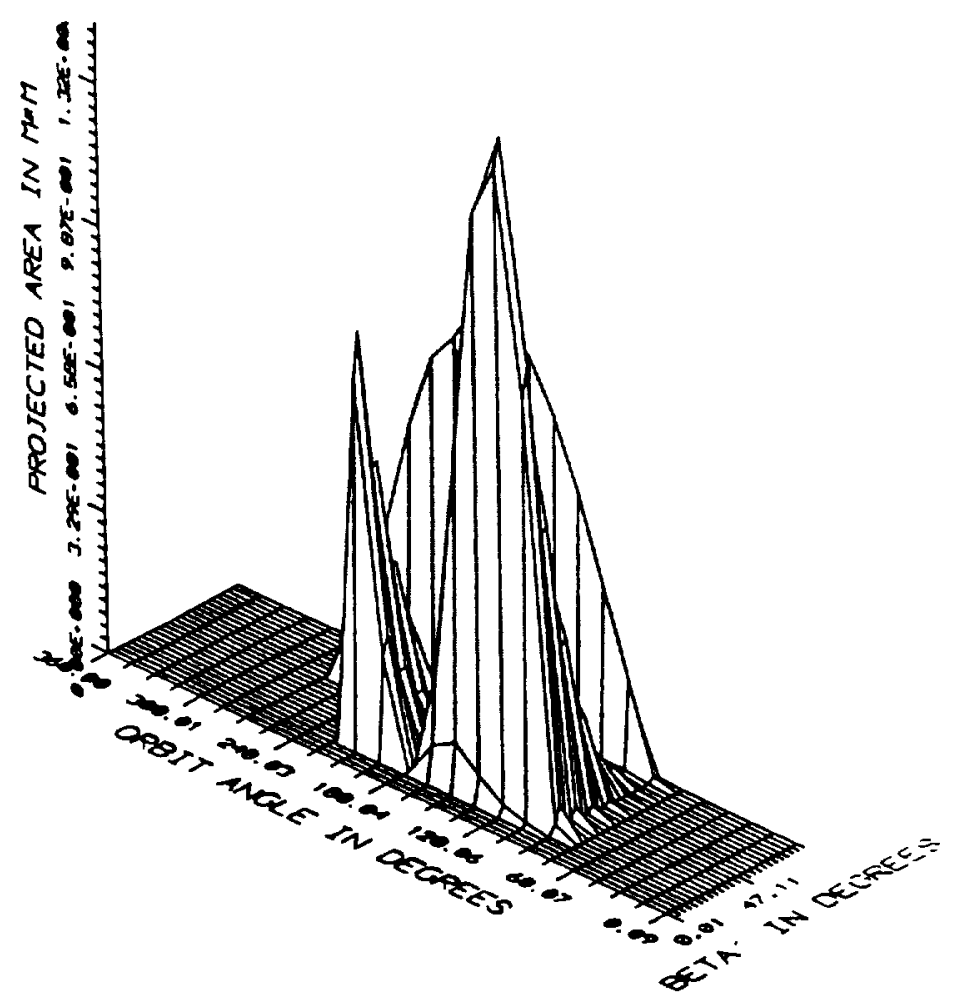

Figure 11. Y+ Plate Projected Area in the Sun direction

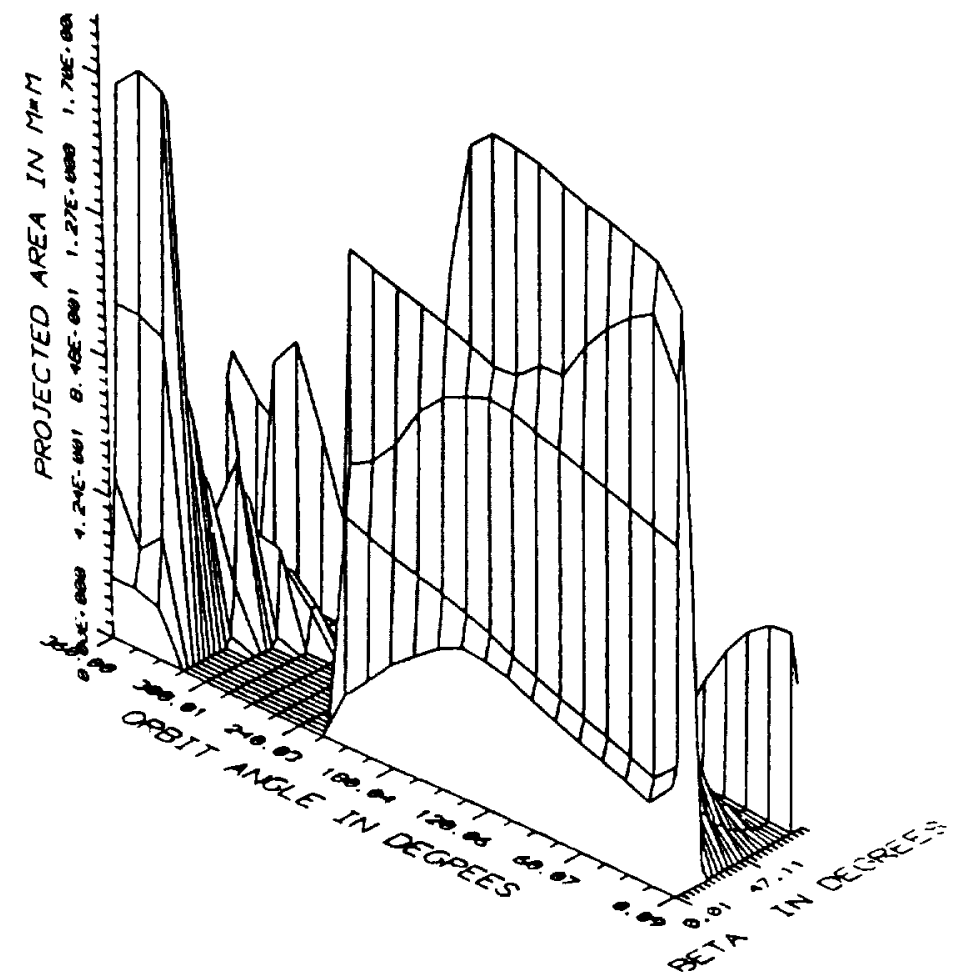

Figure 12. Y-Plate Projected Area in the Sun direction 


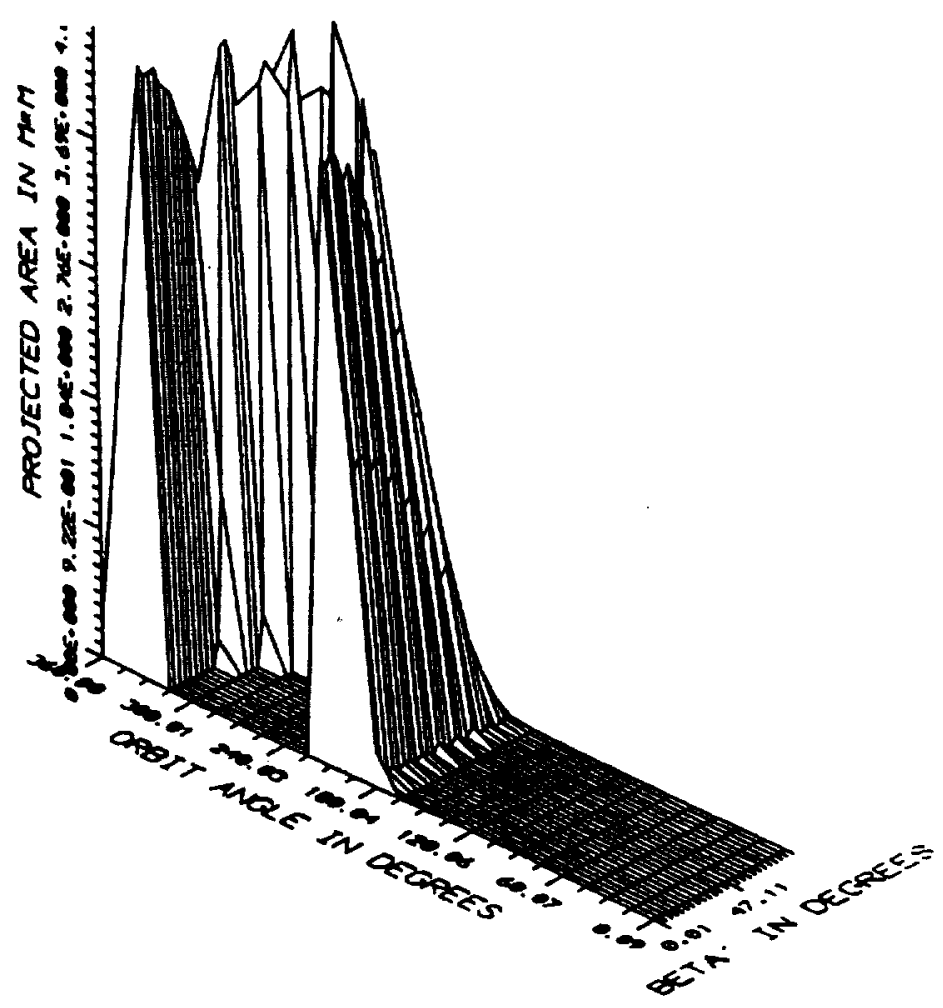

Figure 13. Z+ Plate Projected Area in Sun Direction

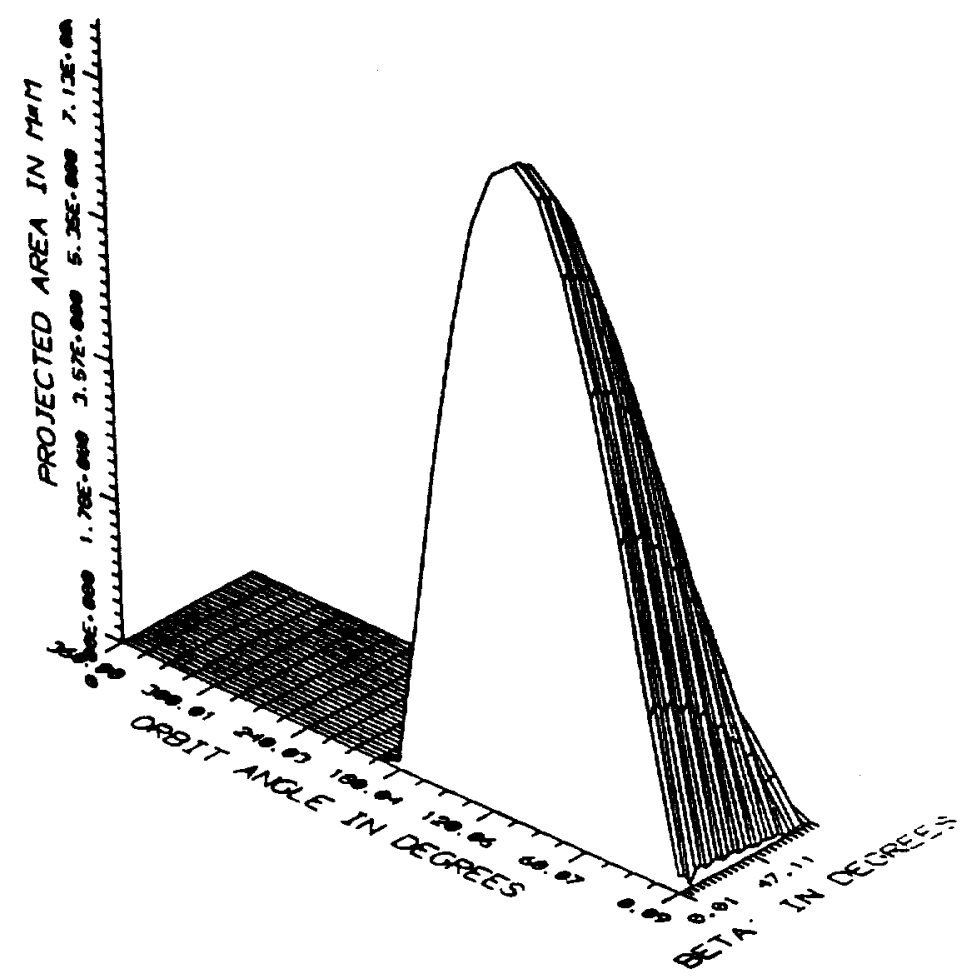

Figure 14. Z- Plate Projected Area in Sun Direction 


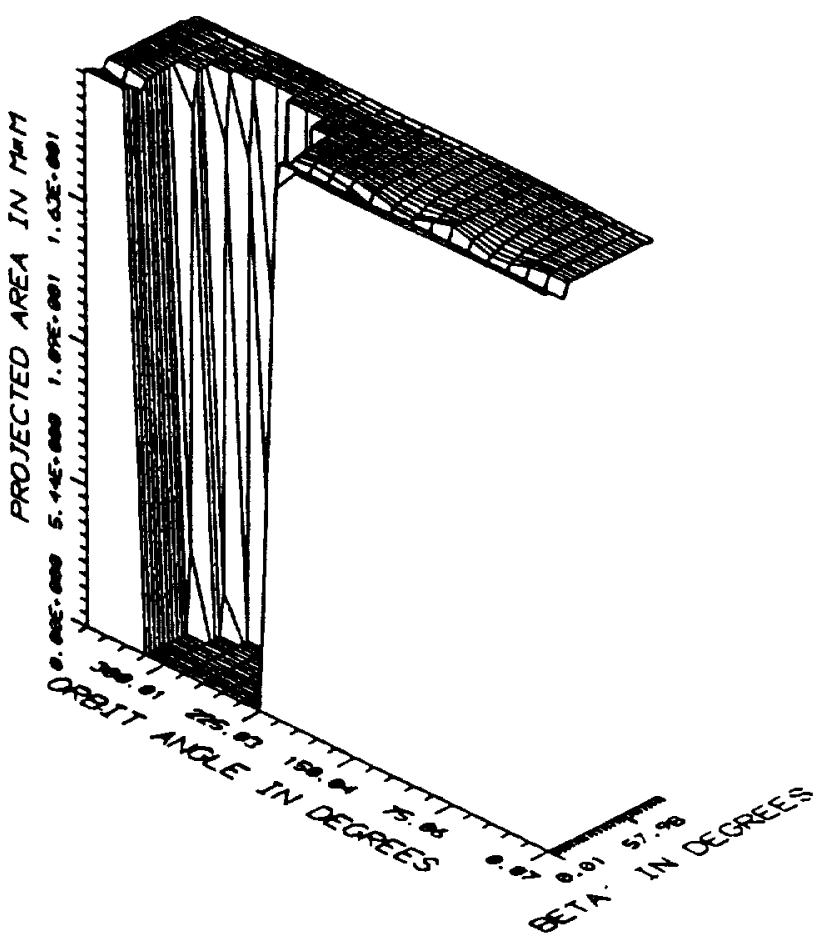

Figure 15. Solar Array Projected Area in Sun Direction 


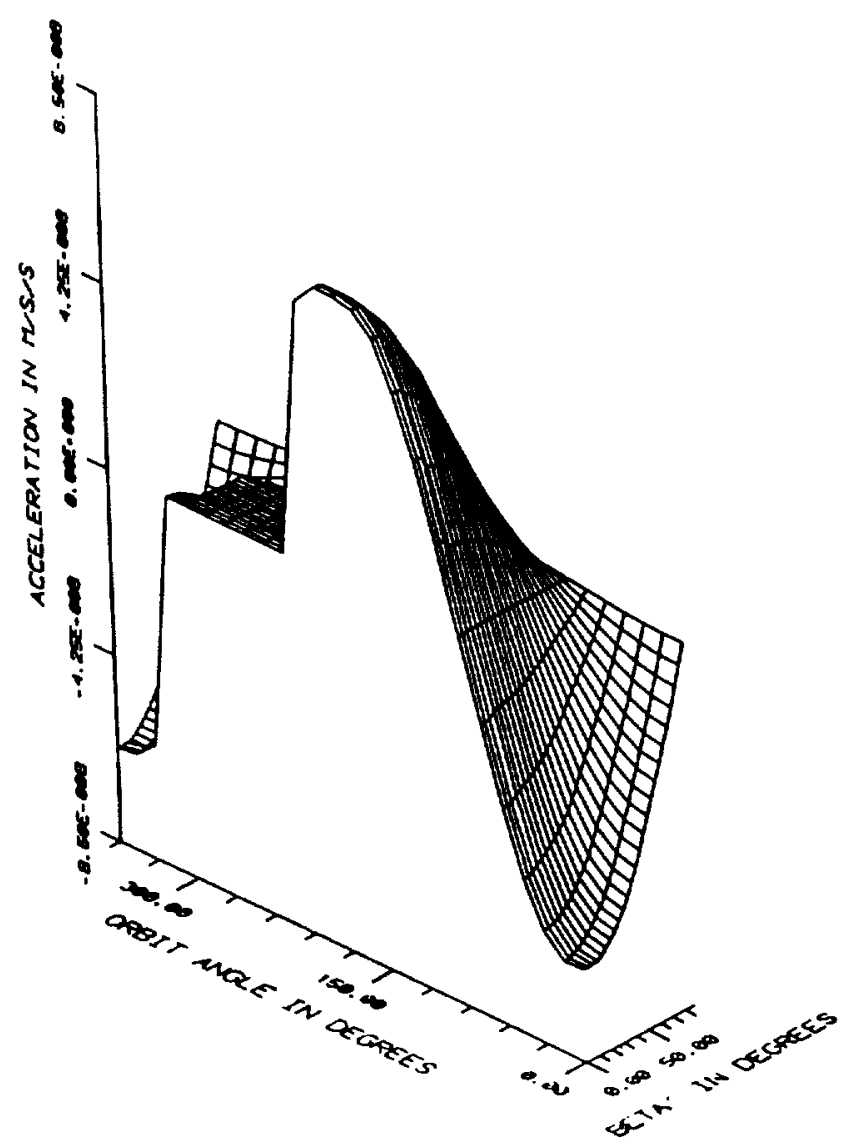

Figure 16. Micro-Model Solar Radiation Pressure Along Track Acceleration

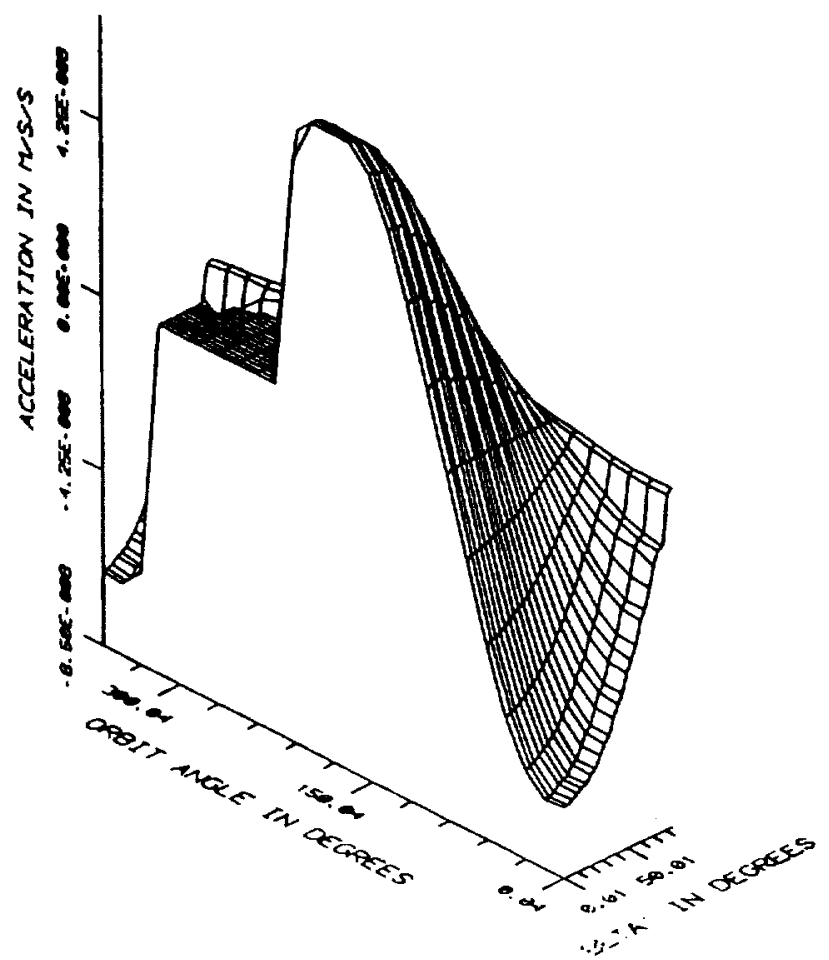

Figure 17. Macro-Model Solar Radiation Pressure Along Track Acceleration 


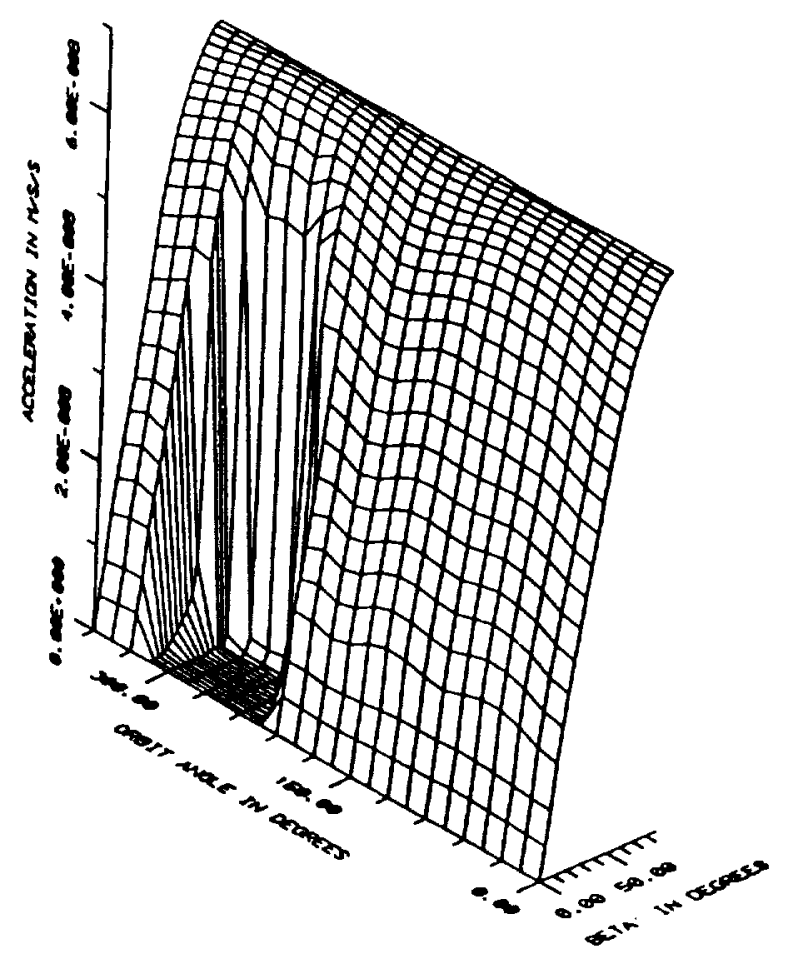

Figure 18. Micro-Model Solar Radiation Pressure Cross Track Acceleration

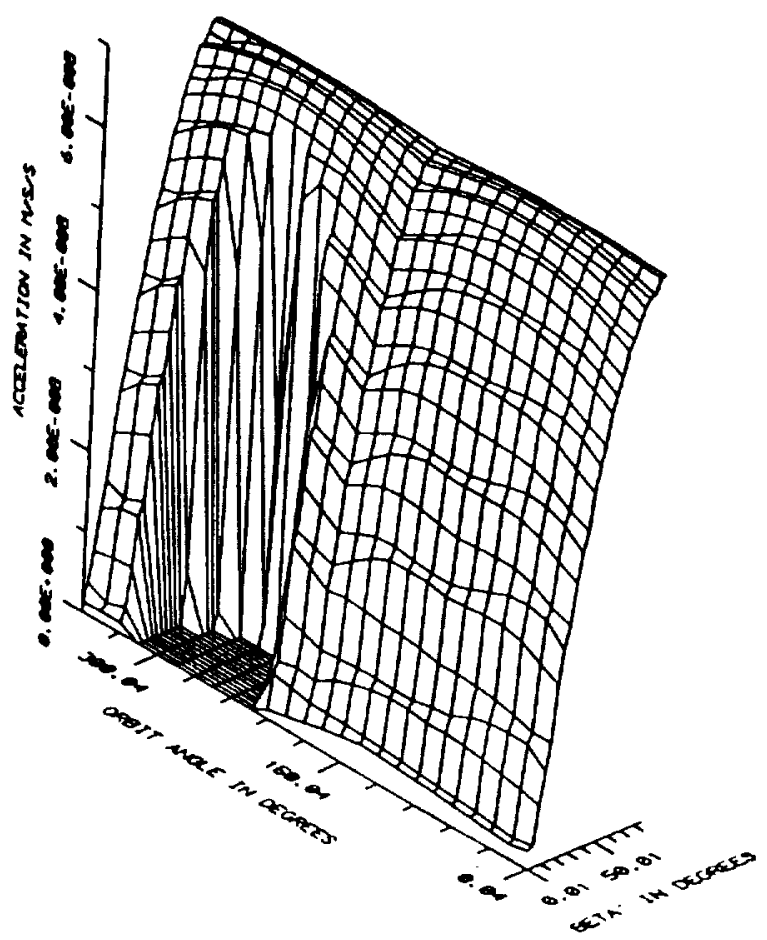

Figure 19. Macro-Model Solar Radiation Pressure Cross Track Acceleration 


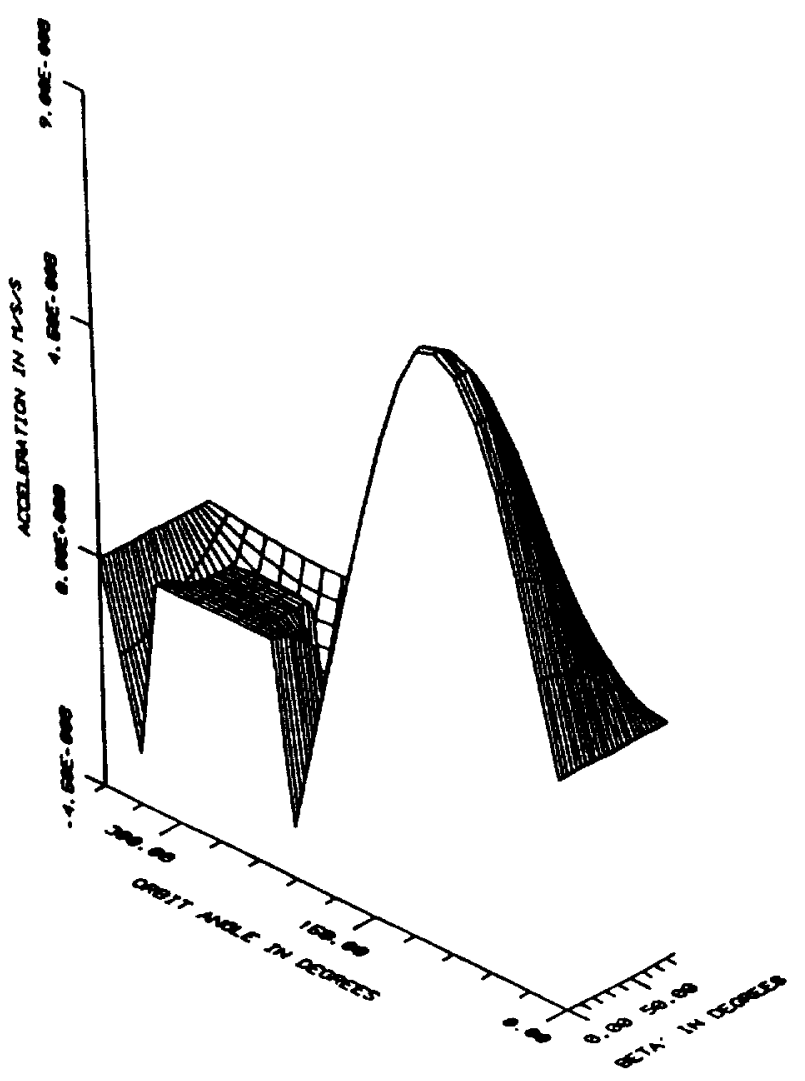

Figure 20. Micro-Model Solar Radiation Pressure Radial Acceleration

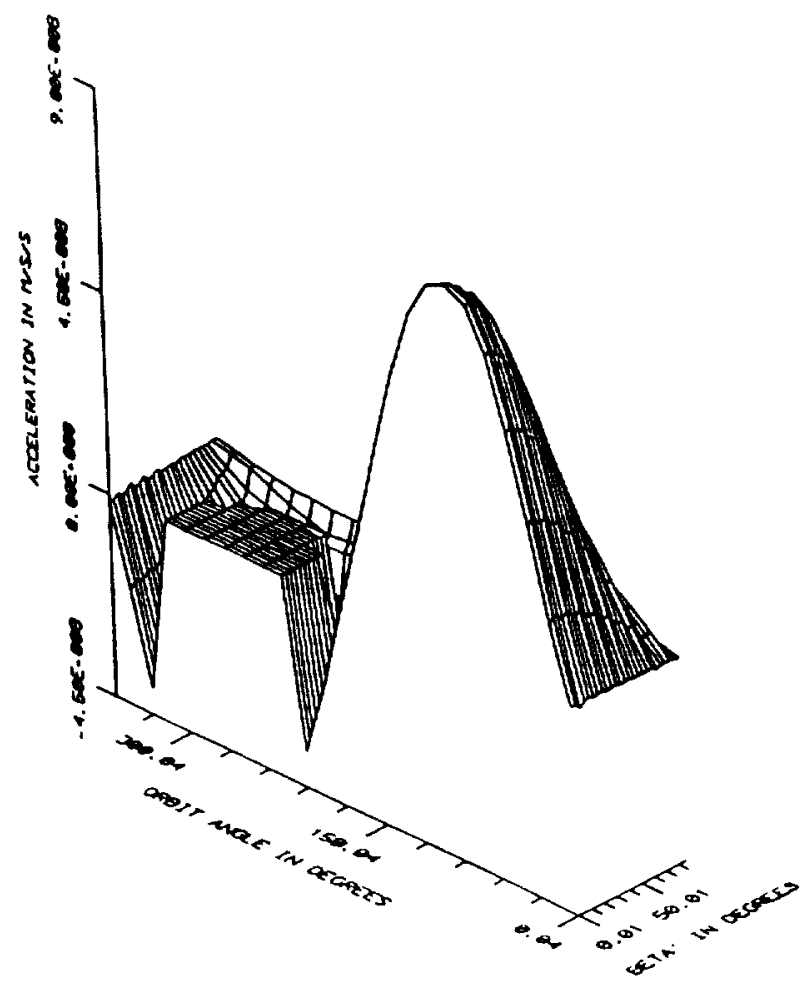

Figure 21. Macro-Model Solar Radiation Pressure Radial Acceleration 


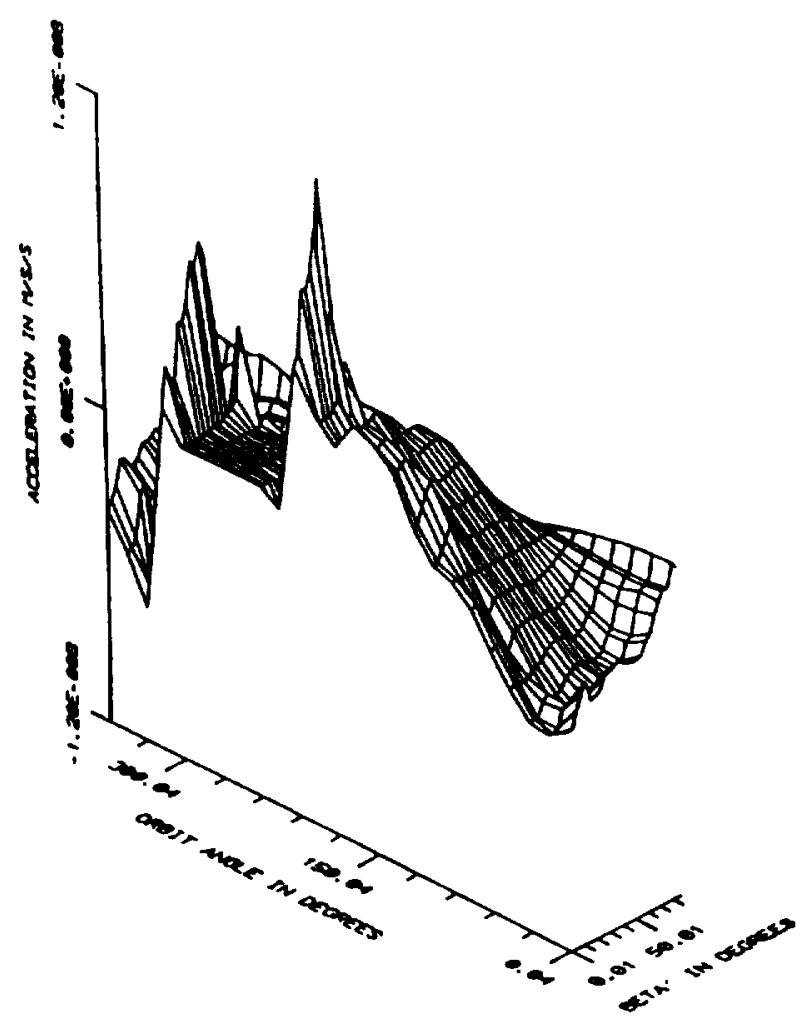

Figure 22. Along Track Solar Radiation Pressure Acceleration Residuals. (Macro-Micro) Mean = -3.8E-11; RMS = 4.3E-9

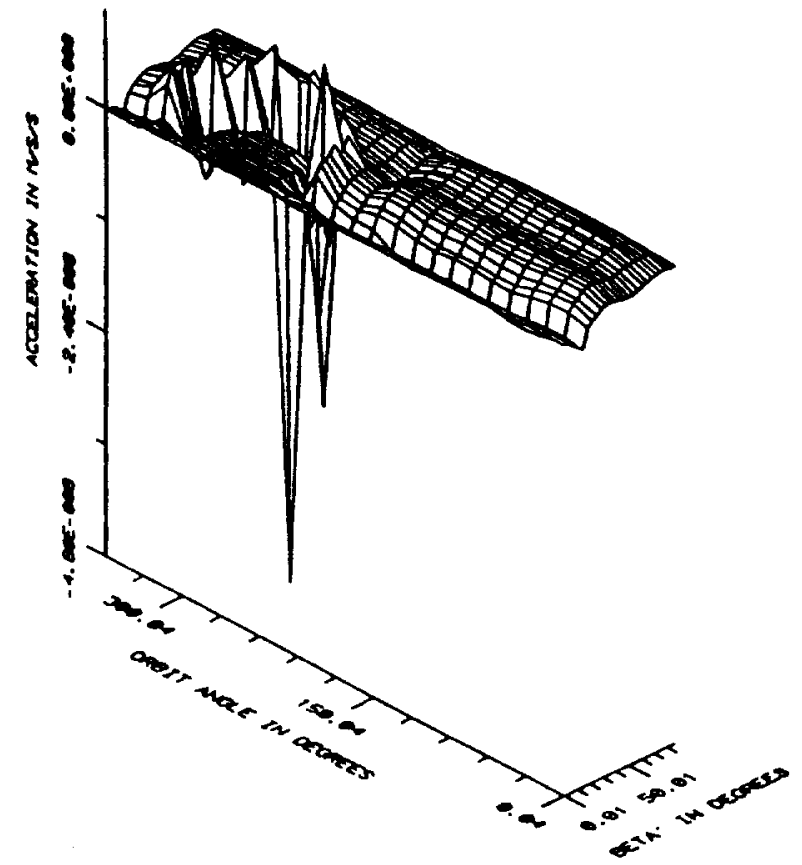

Figure 23. Cross Track Solar Radiation Pressure Acceleration Residuals. (Macro-Micro) Mean = 1.7E-9; RMS = 4.3E-9 


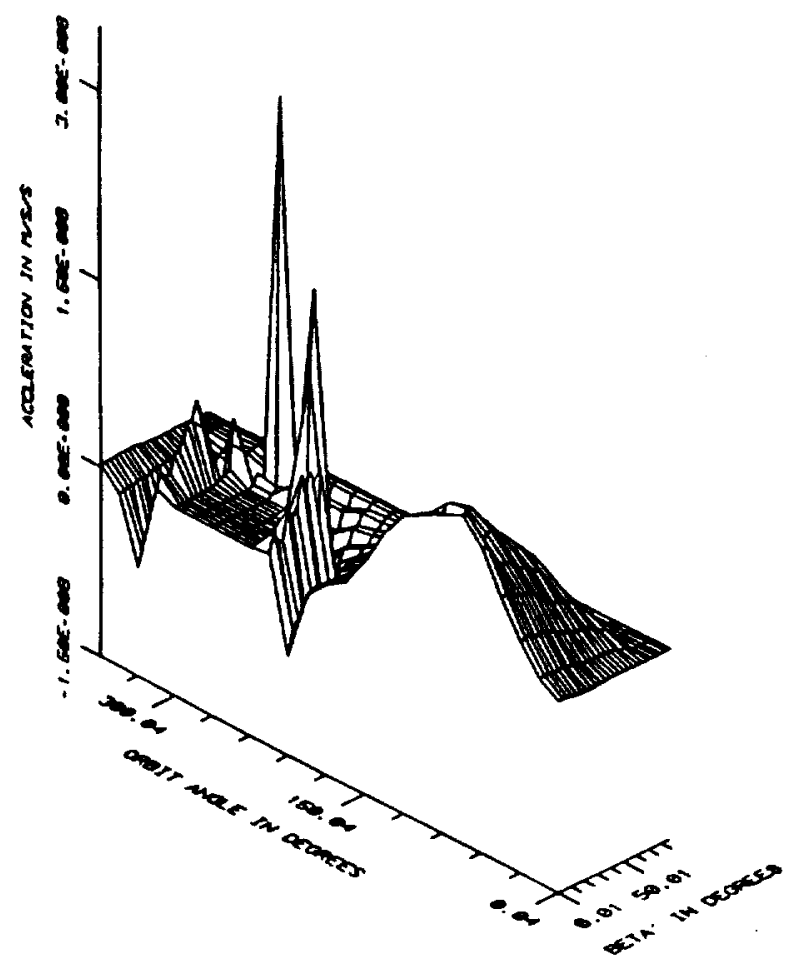

Figure 24. Radial Solar Radiation Pressure Acceleration Residuals. (Macro-Micro) Mean = 1.3E-9; RMS = 4.9E-9 


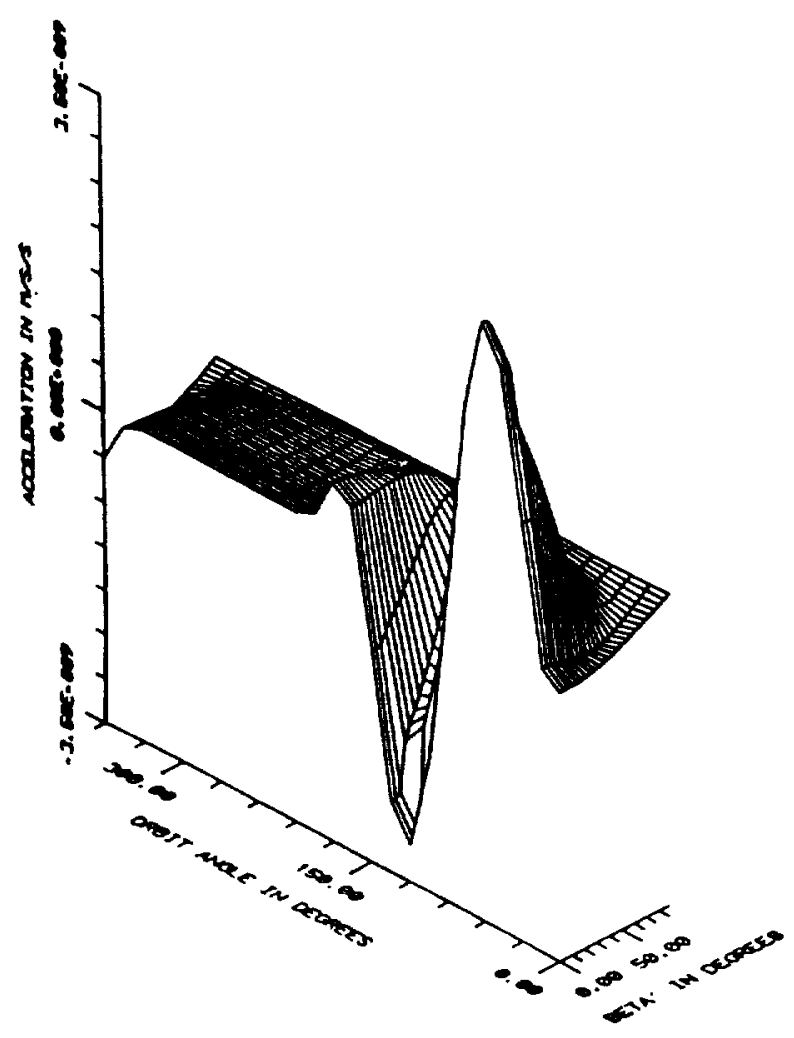

Figure 25. Micro-Model Earth Albedo Along Track Acceleration

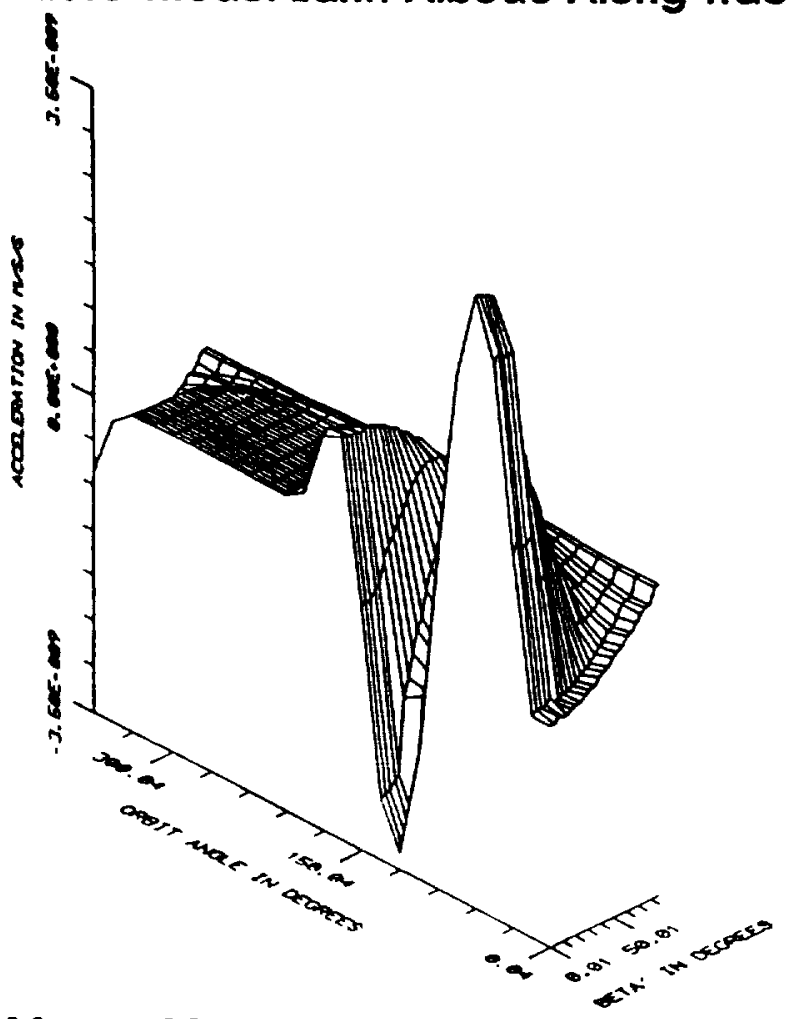

Figure 26. Macro-Model Earth Albedo Along Track Acceleration 


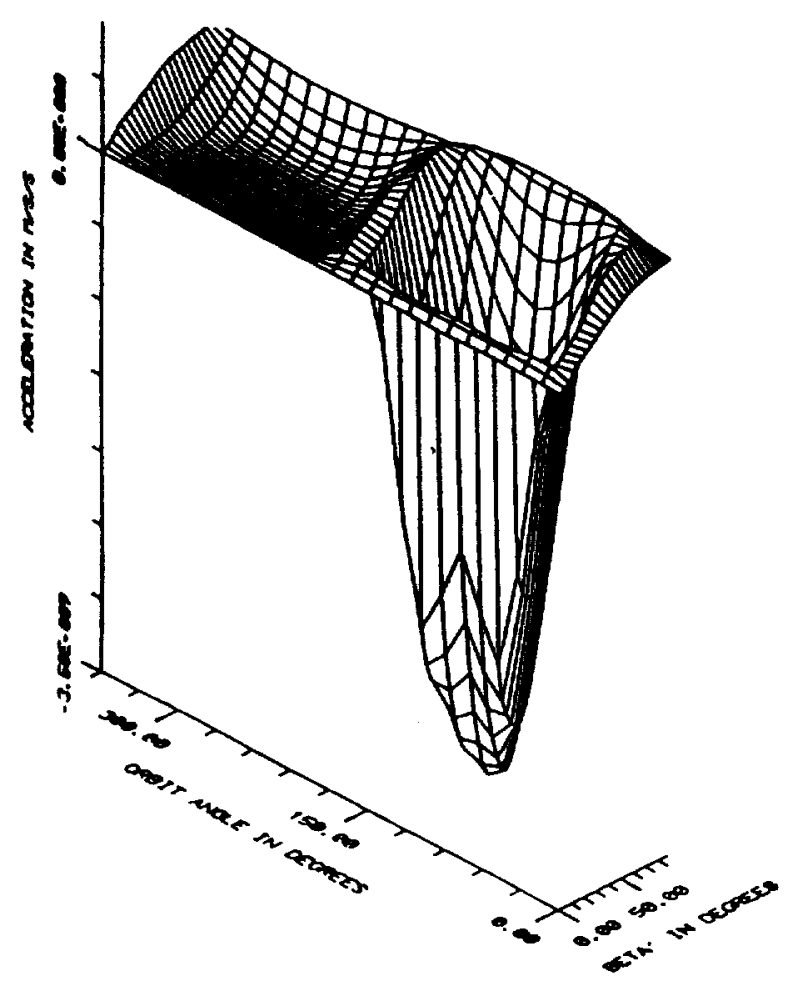

Figure 27. Micro-Model Earth Albedo Cross Track Acceleration

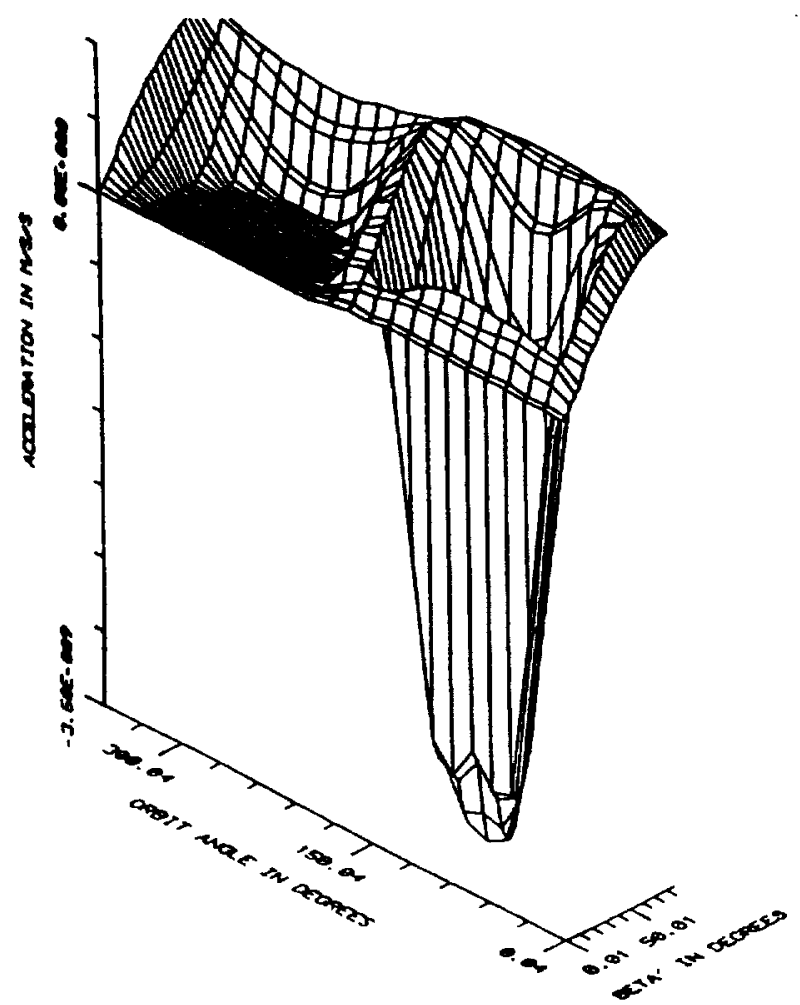

Figure 28. Macro-Model Earth Albedo Cross Track Acceleration 


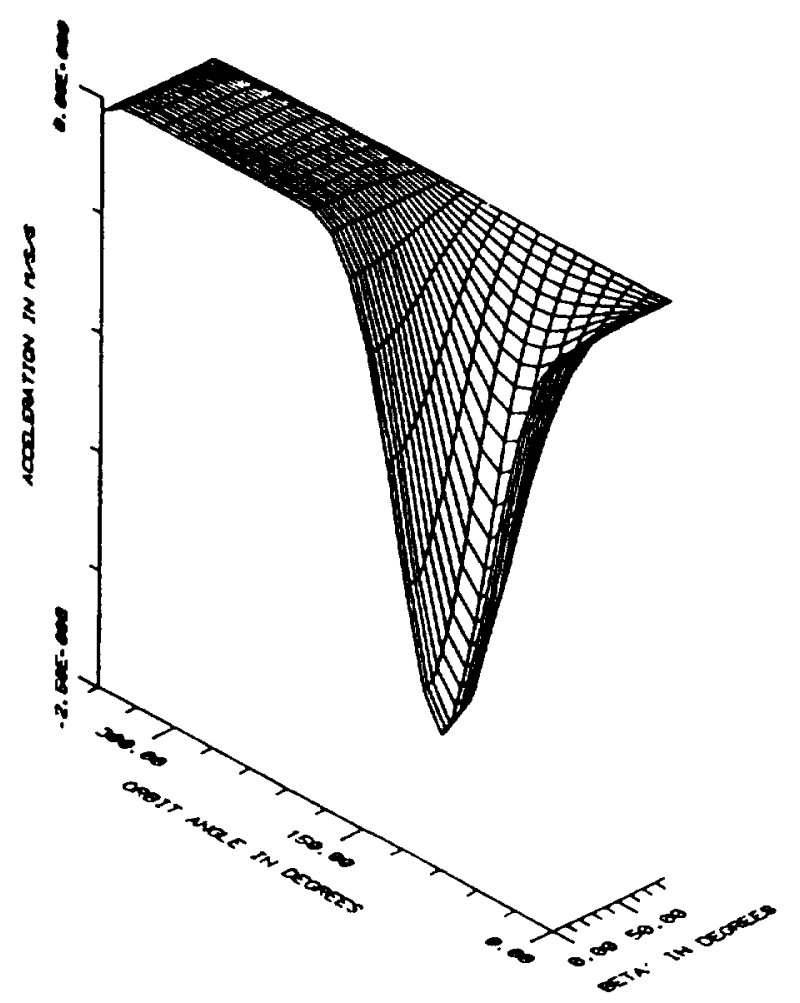

Figure 29. Micro-Model Earth Albedo Radial Acceleration

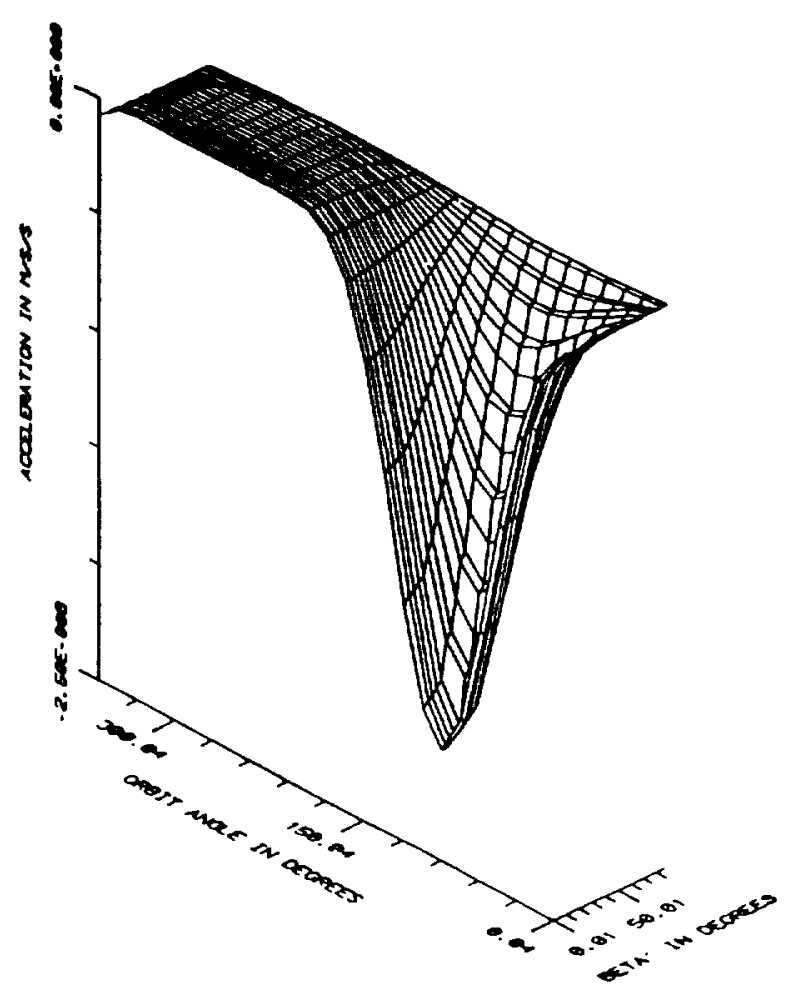

Figure 30. Macro-Model Earth Albedo Radial Acceleration 


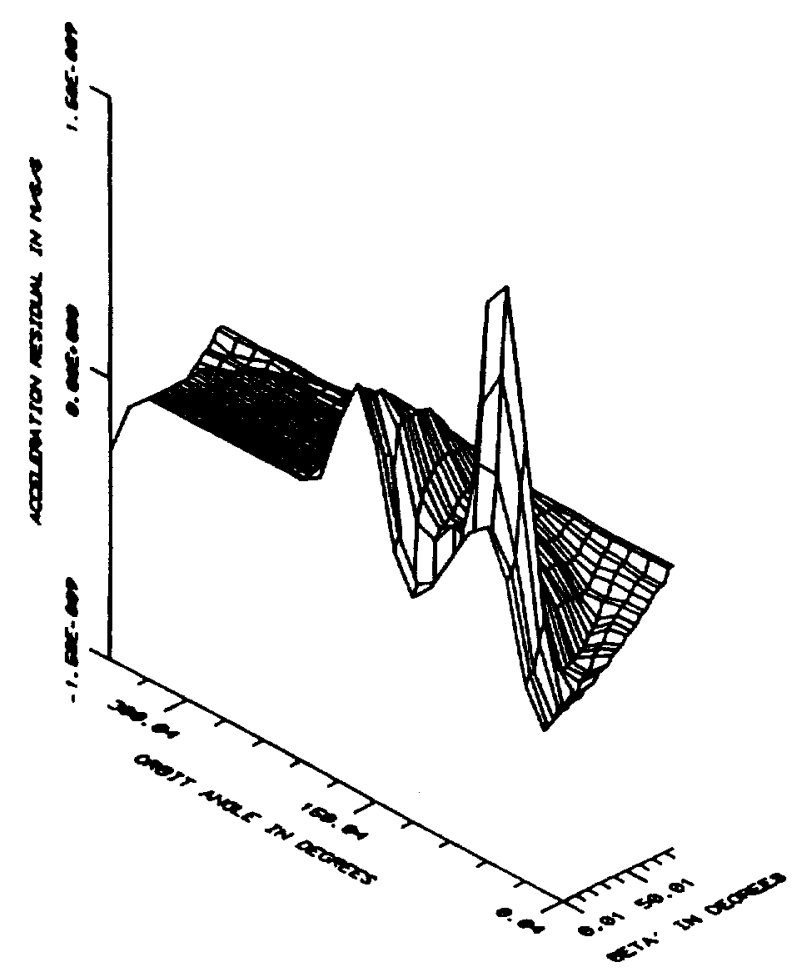

Figure 31. Along Track Earth Albedo Acceleration Residuals. (Macro-Micro)

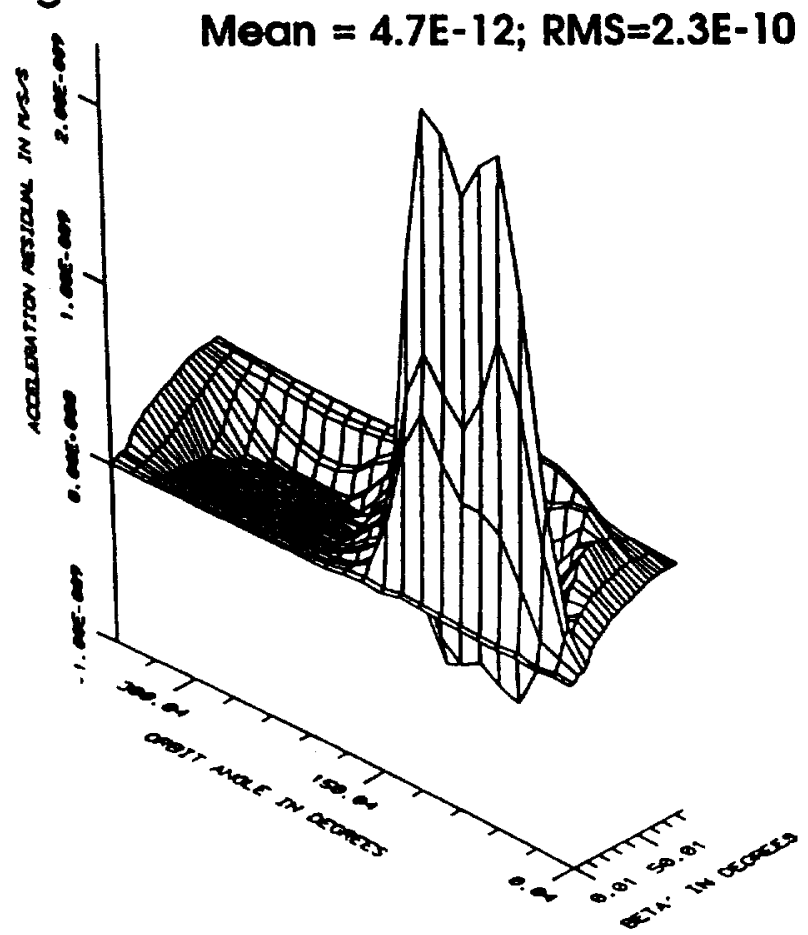

Figure 32. Cross Track Earth Albedo Acceleration Residuals. (Macro-Micro) Mean = 1.9E-10; RMS=3.3E-10 


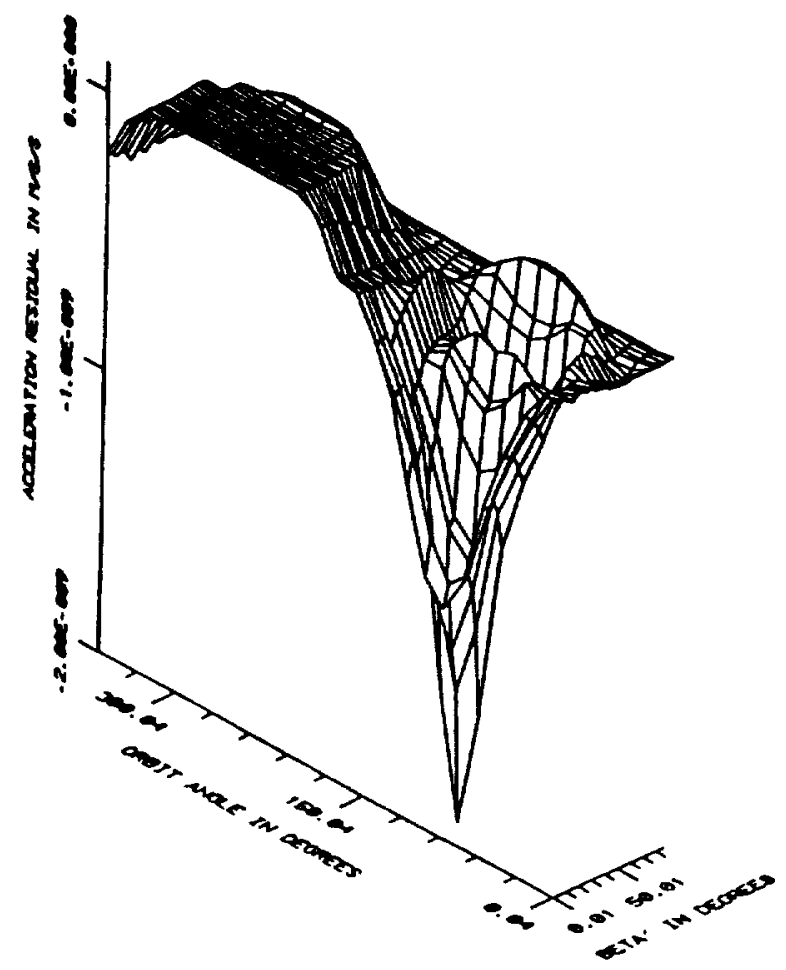

Figure 33. Radial Earth Albedo Acceleration Residuals. (Macro-Micro) Mean $=2.2 E-10 ;$ RMS $=2.4 E-10$ 


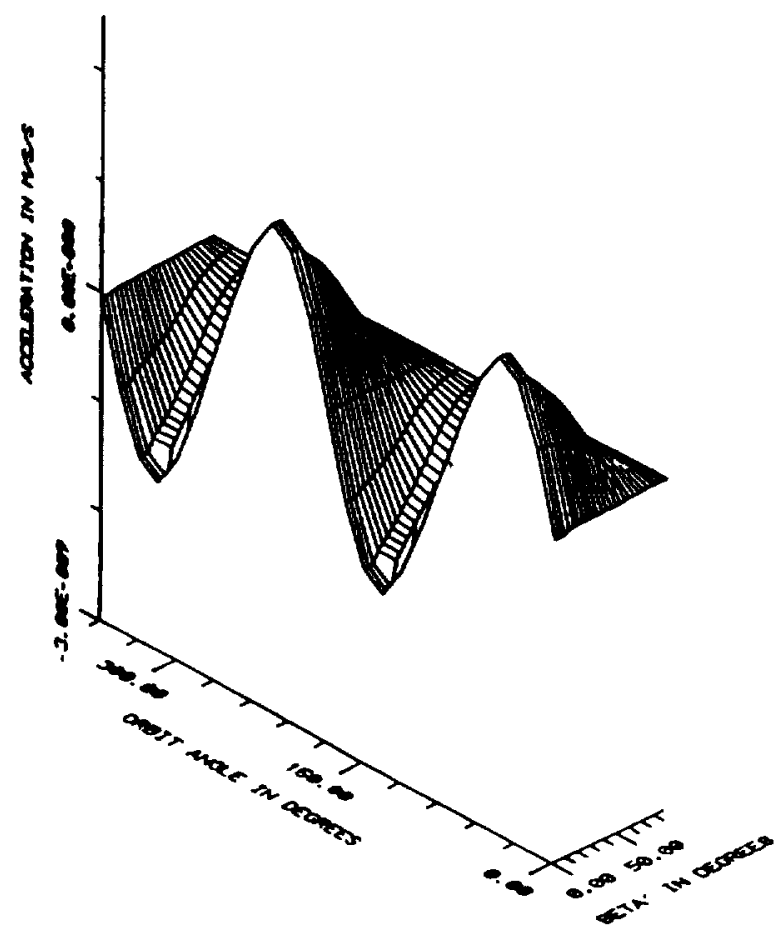

Figure 34. Micro-Model Earth IR Along Track Acceleration

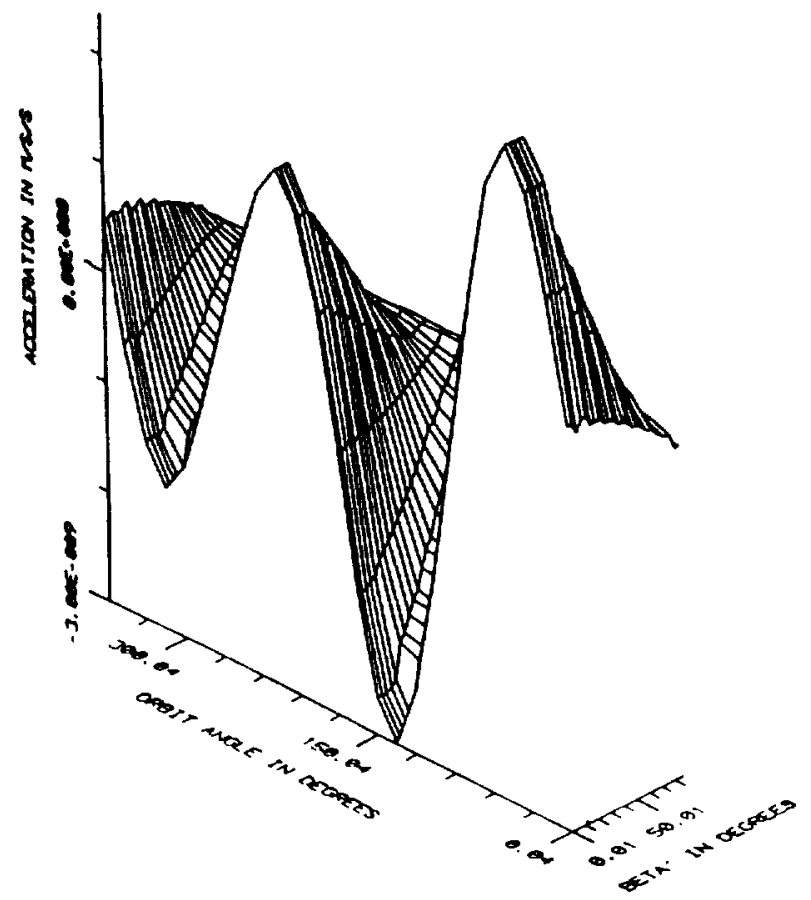

Figure 35. Macro-Model Earth IR Along Track Acceleration 


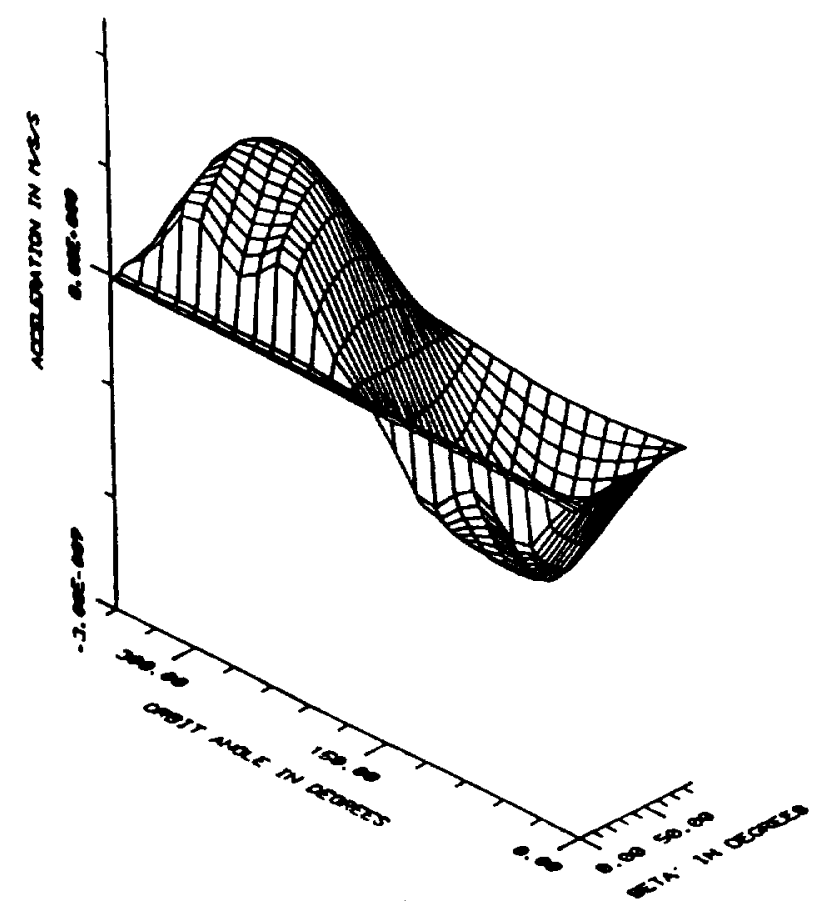

Figure 36. Micro-Model Earth IR Cross Track Acceleration

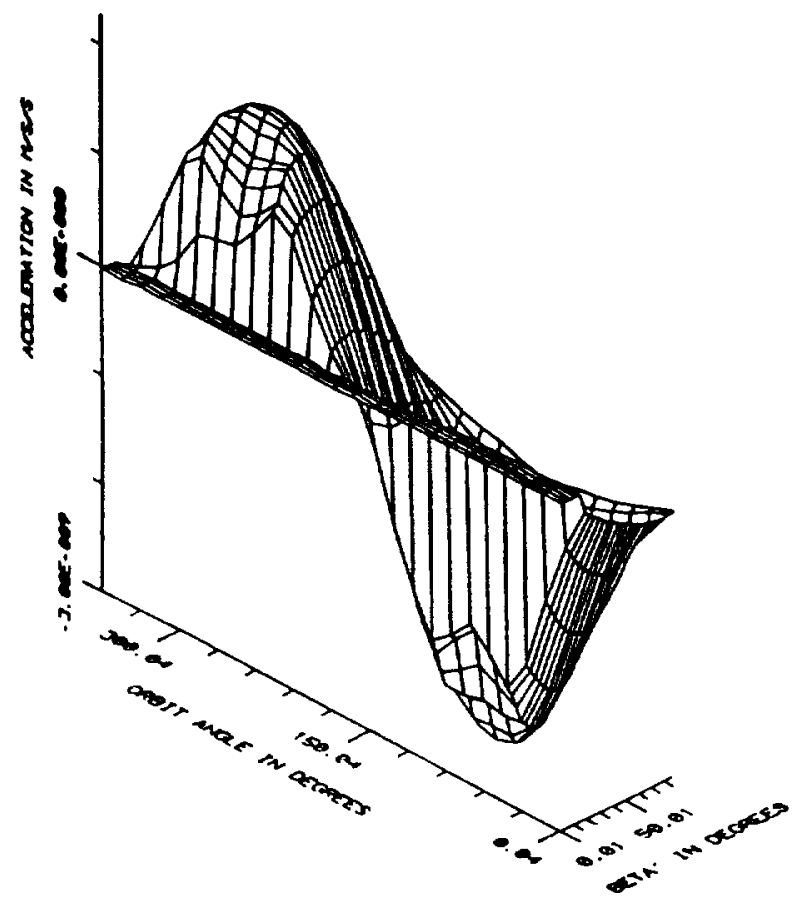

Figure 37. Macro-Model Earth IR Cross Track Acceleration 


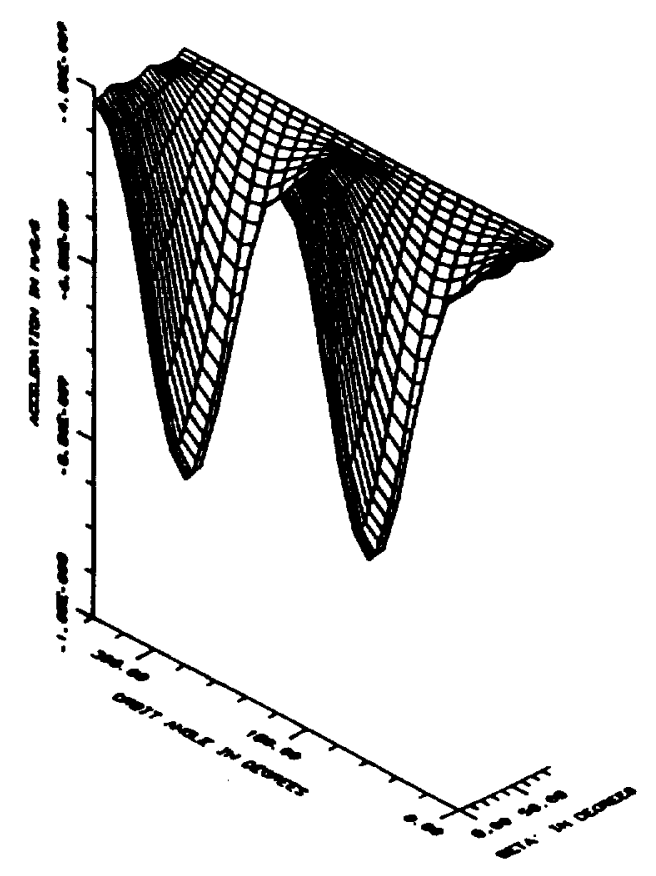

Figure 38. Micro-Model Earth IR Radial Acceleration

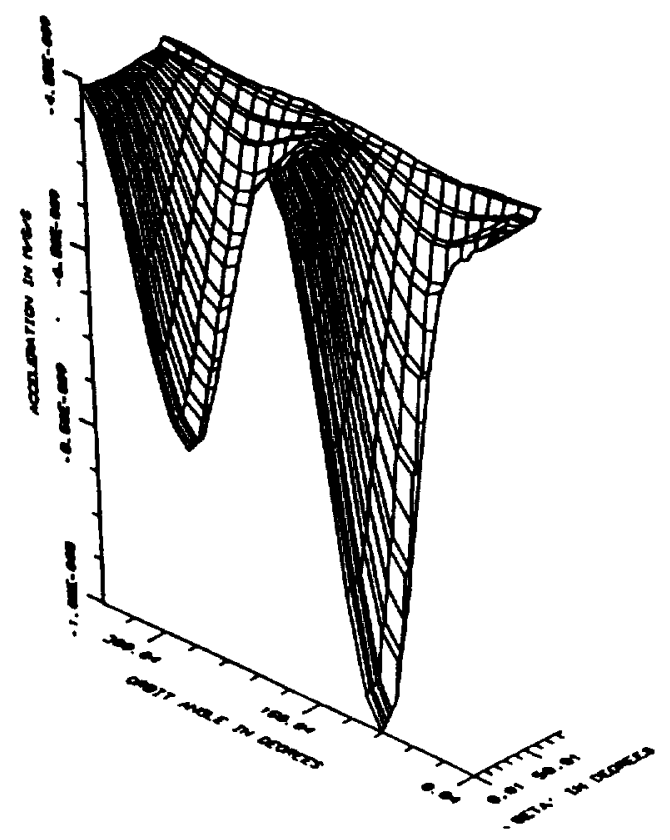

Figure 39. Macro-Model Earth IR Radial Acceleration 


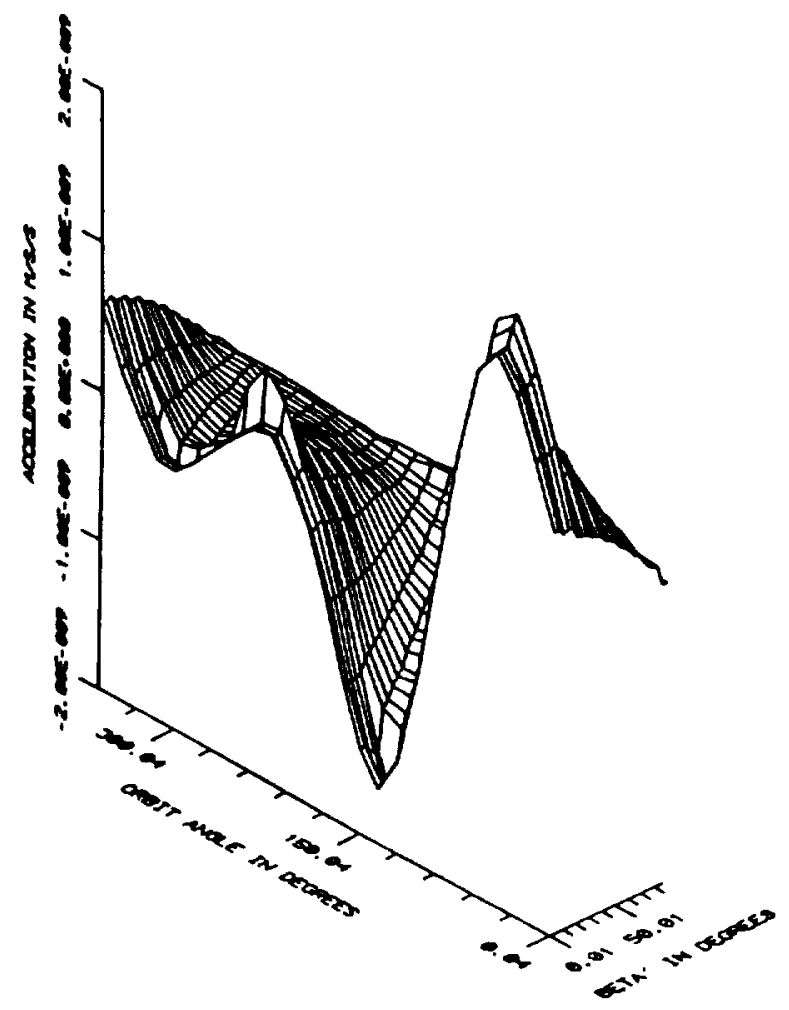

Figure 40. Along Track Earth IR Acceleration Residuals.(Macro-Micro) Mean $=8.5 \mathrm{E}-12 ; \mathrm{RMS}=5.4 \mathrm{E}-10$

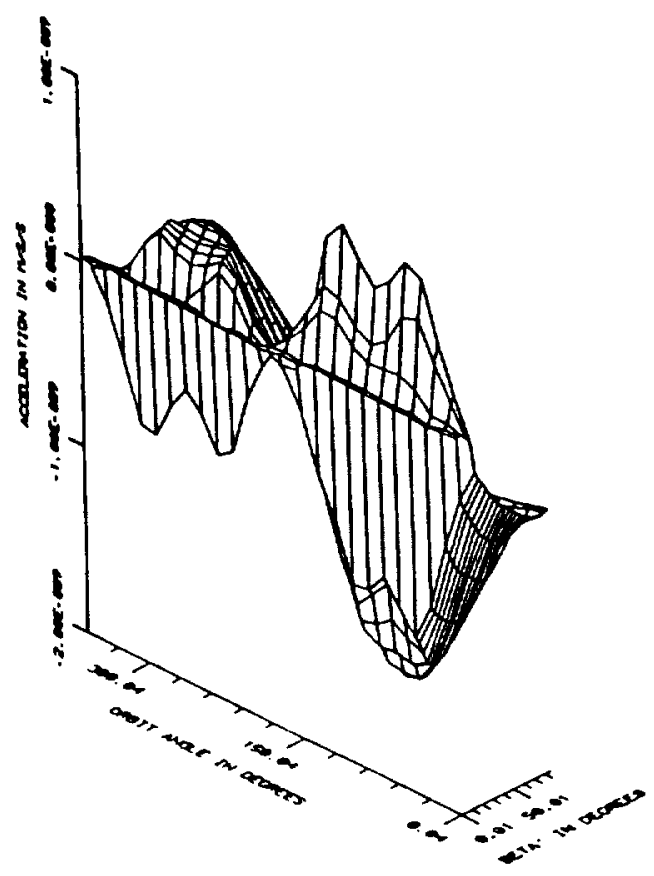

Figure 41. Cross Track Earth IR Acceleration Residuals. (Macro-Micro) Mean = 4.4E-10; RMS = 5.3E-10 


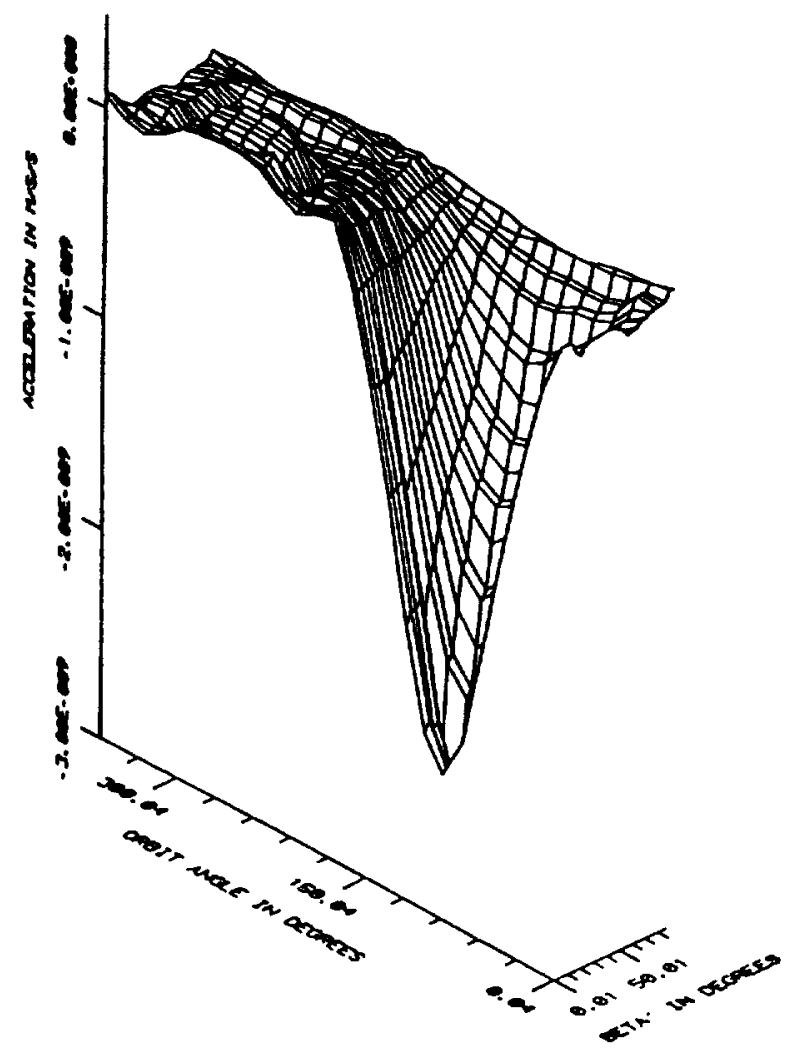

Figure 42. Radial Earth IR Acceleration Residuals. (Macro-Micro) Mean $=-2.8 E-10 ;$ RMS $=5.4 E-10$ 


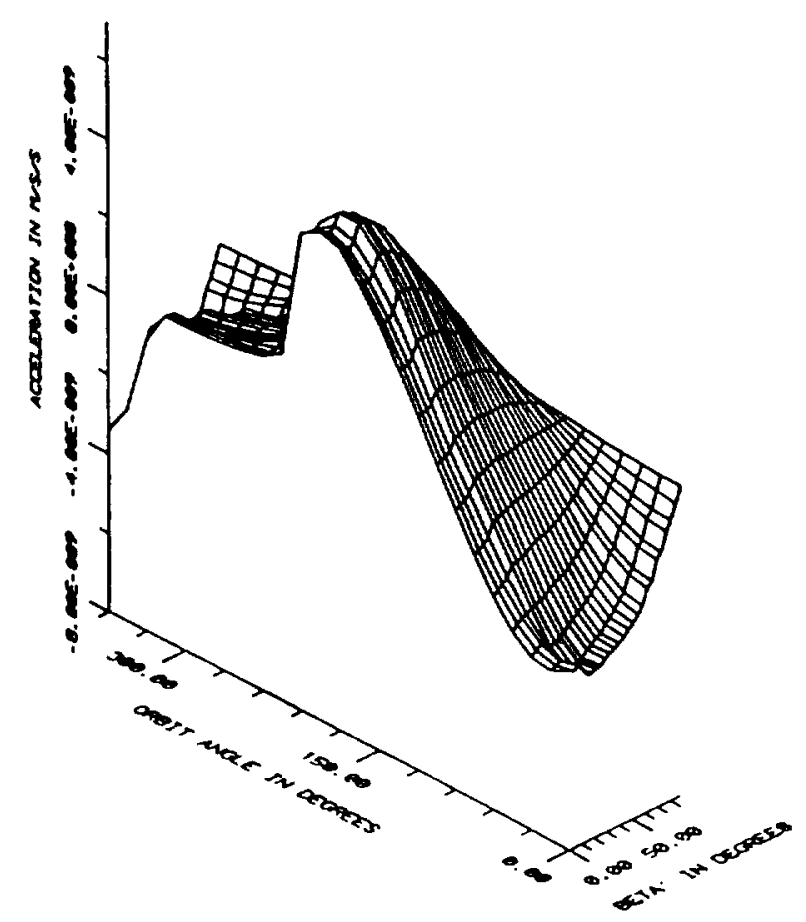

Figure 43. Micro-Model Thermal Imbalance Along Track Acceleration

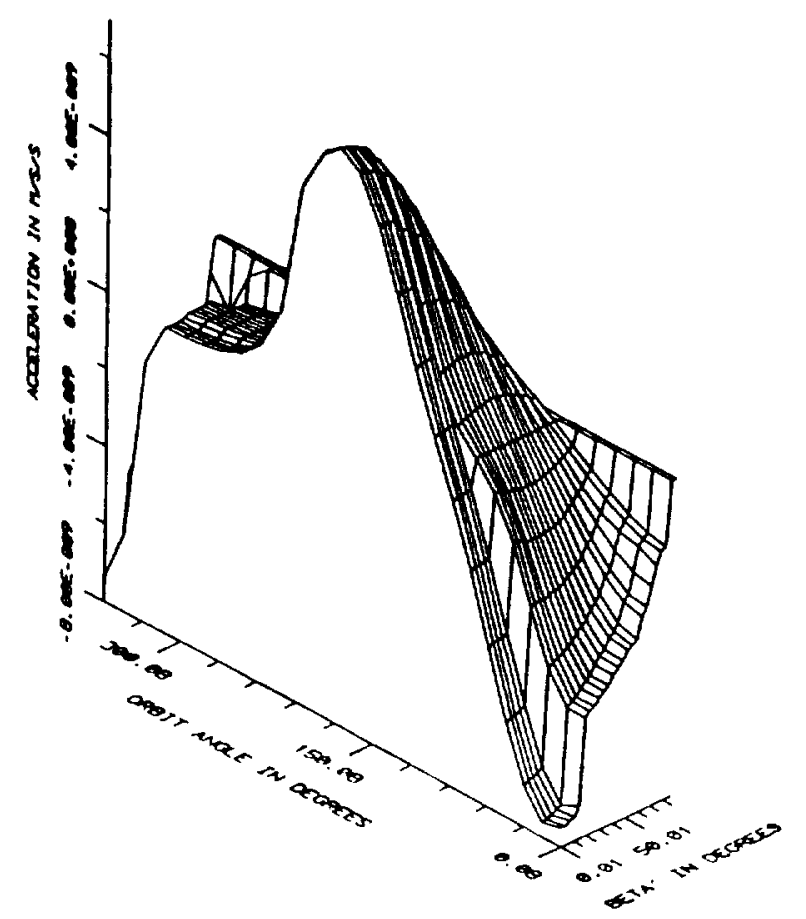

Figure 44. Macro-Model Thermal Imbalance Along Track Acceleration 


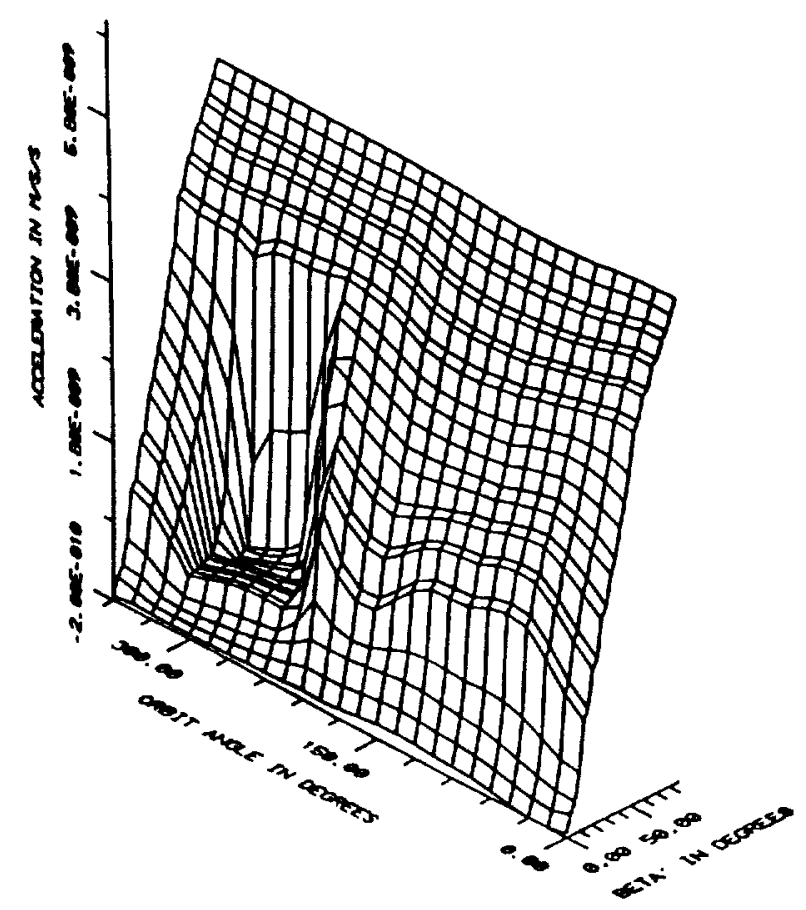

Figure 45. Micro-Model Thermal Imbalance Cross Track Acceleration

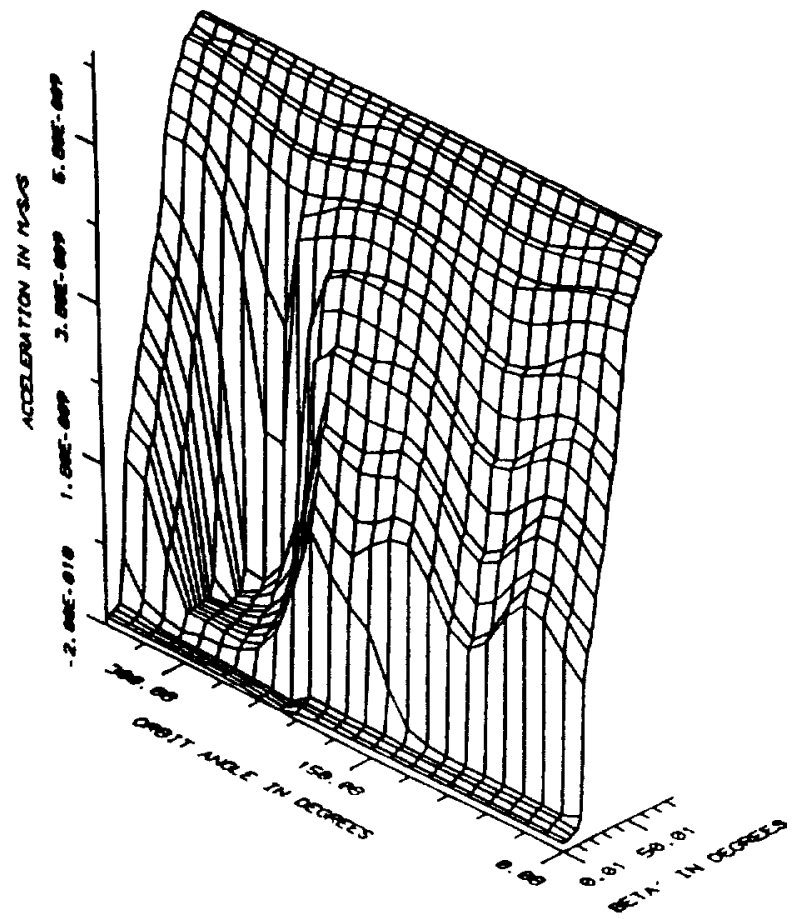

Figure 46. Macro-Model Thermal Imbalance Cross Track Acceleration 


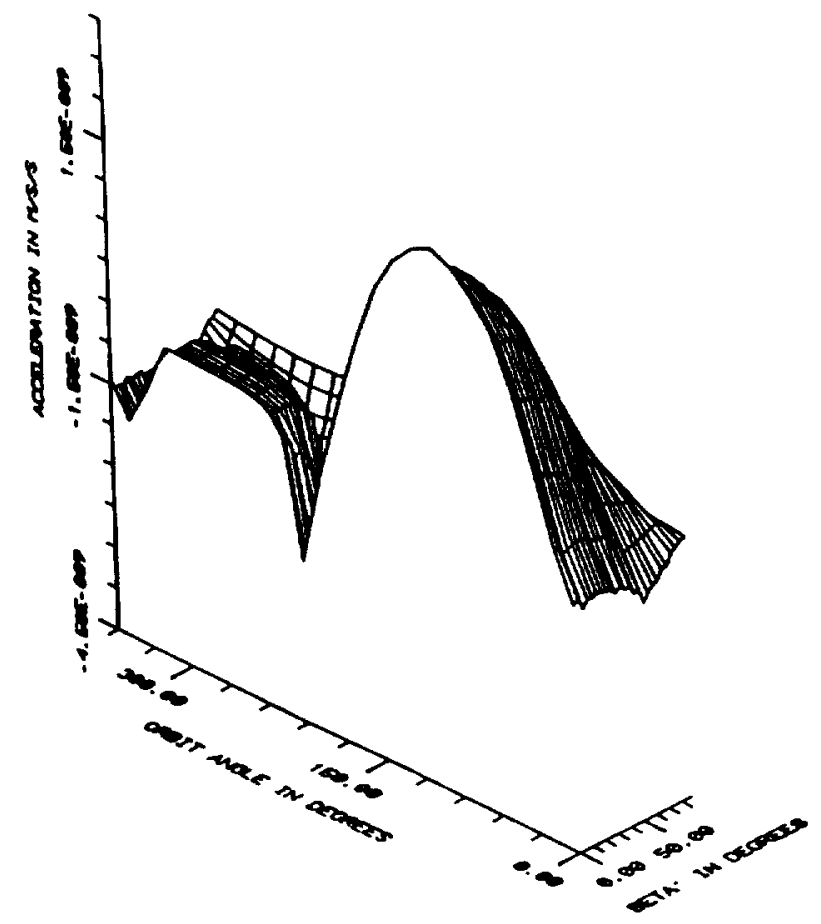

Figure 47. Micro-Model Thermal Imbalance Radial Acceleration

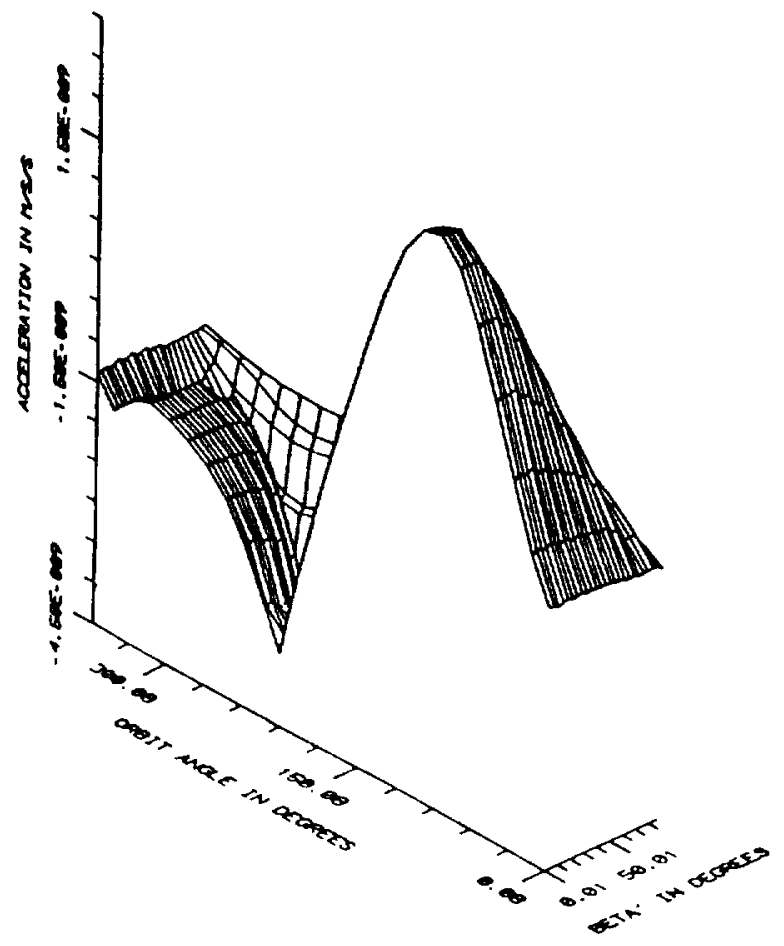

Figure 48. Macro-Model Thermal Imbalance Radial Acceleration 


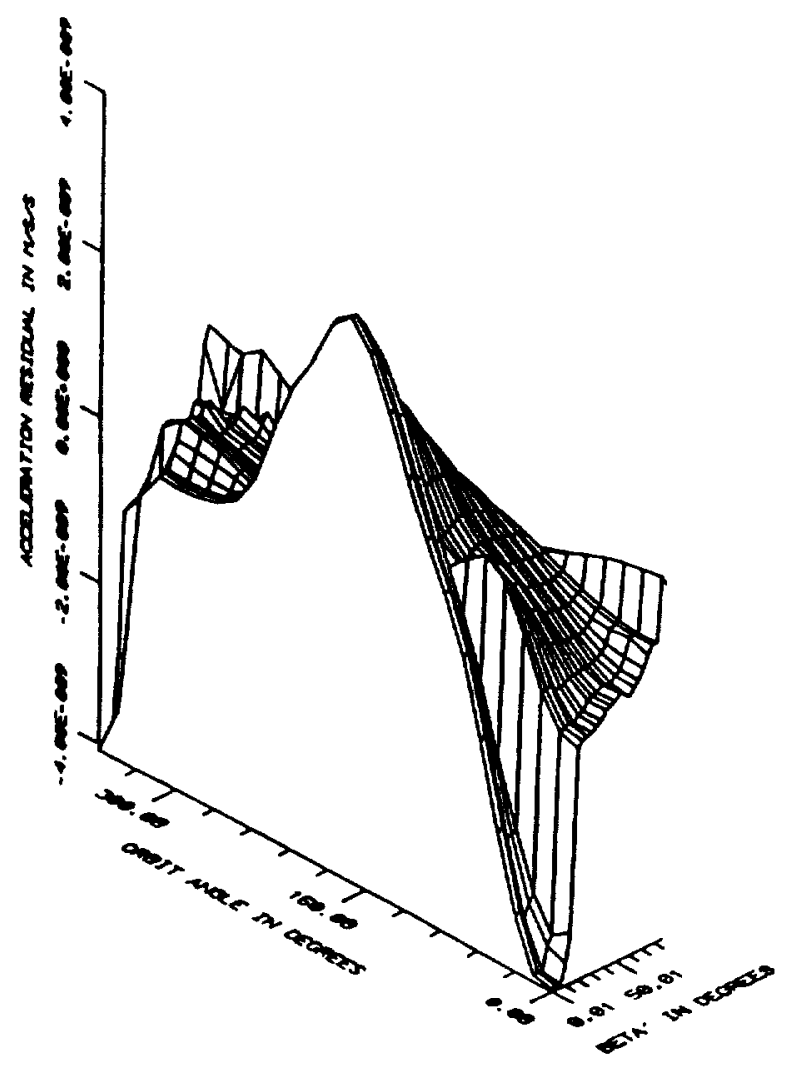

Figure 49. Along Track Thermal Imbalance Acceleration Residuals. (Macro-Micro) Mean = 4.9E-11; RMS = 1.09E-9

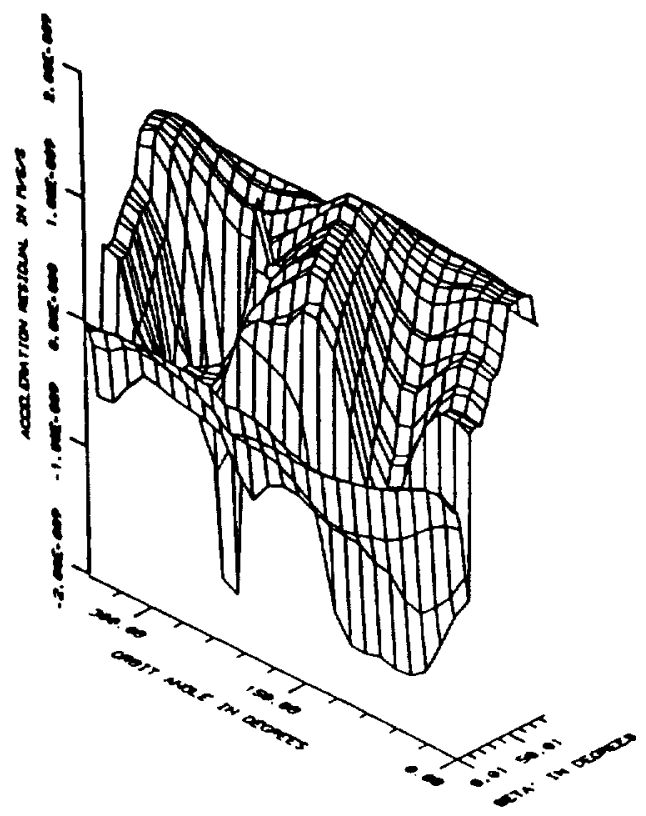

Figure 50. Cross Track Thermal Imbalance Acceleration Residuals. (Macro-Micro) Mean = 5.9E-10; RMS = 7.6E-10 


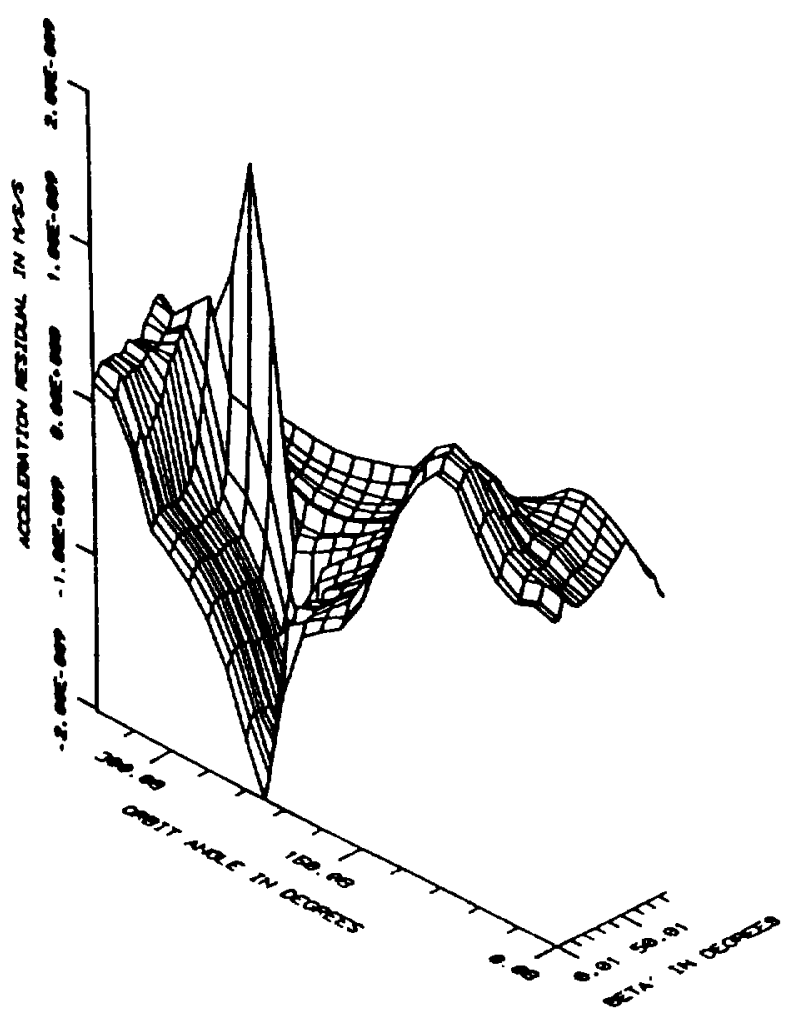

Figure 51. Radial Thermal Imbalance Acceleration Residuals. (Macro-Micro) Mean = 1.6E-10; RMS = 5.5E-10 


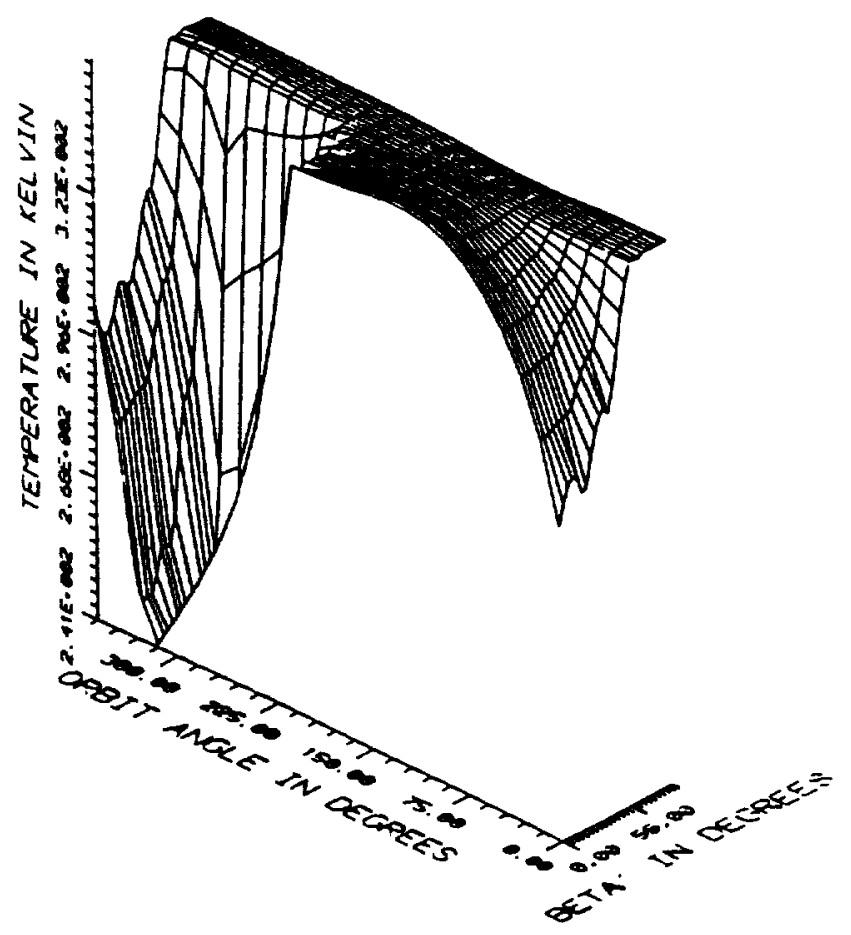

Figure 52. SA+ Micro-Model Temperature Profile

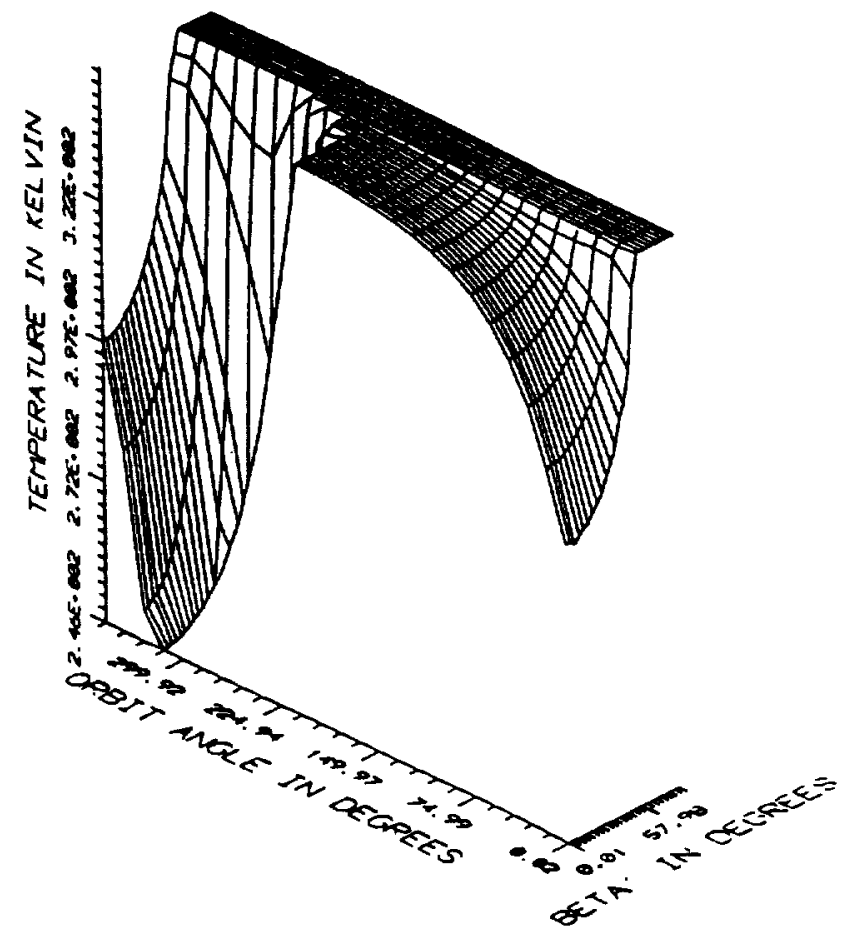

Figure 53. SA+ Macro-Model Temperature Profile 


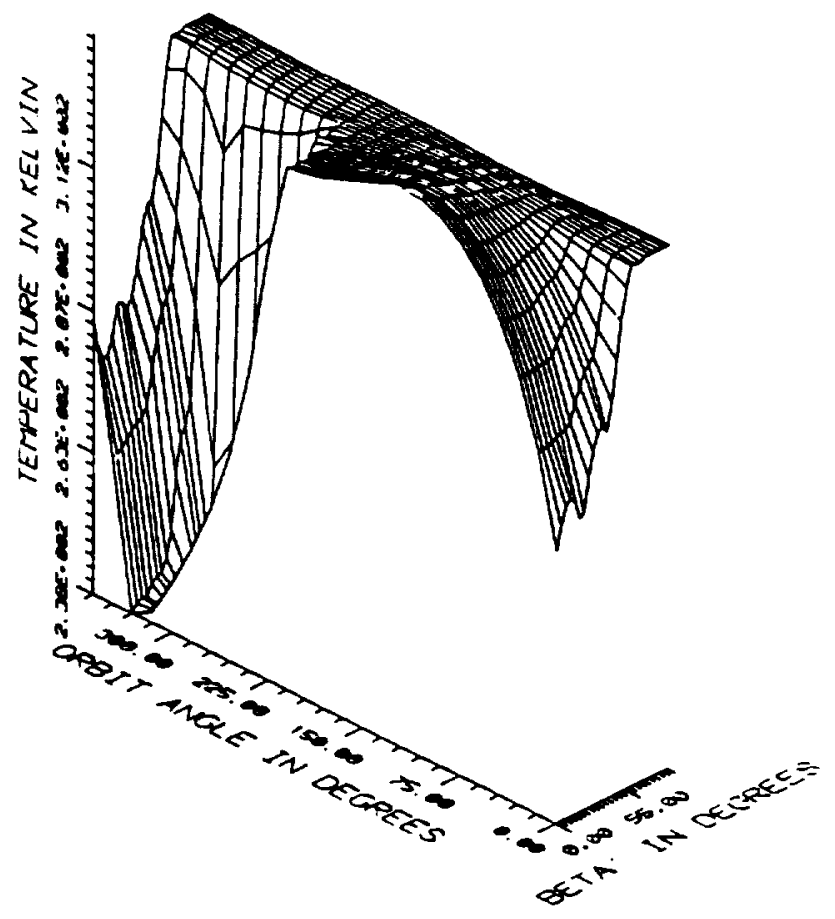

Figure 54. SA-Micro-Model Temperature Profile

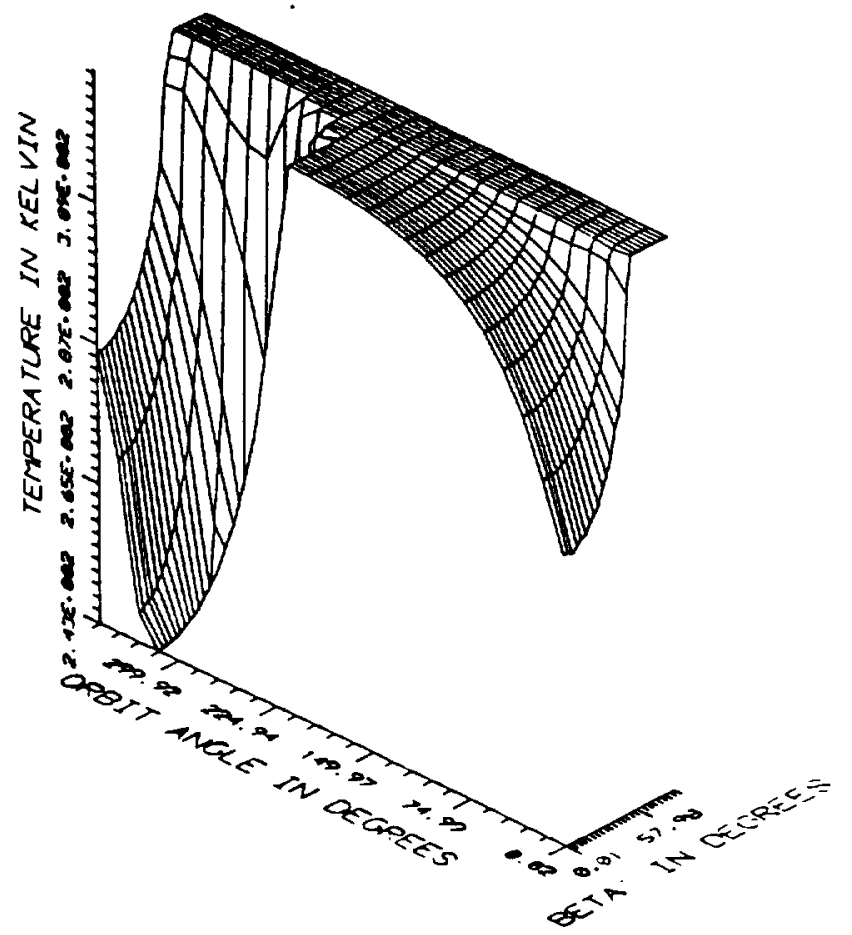

Figure 55. SA-Macro-Model Temperature Profile 


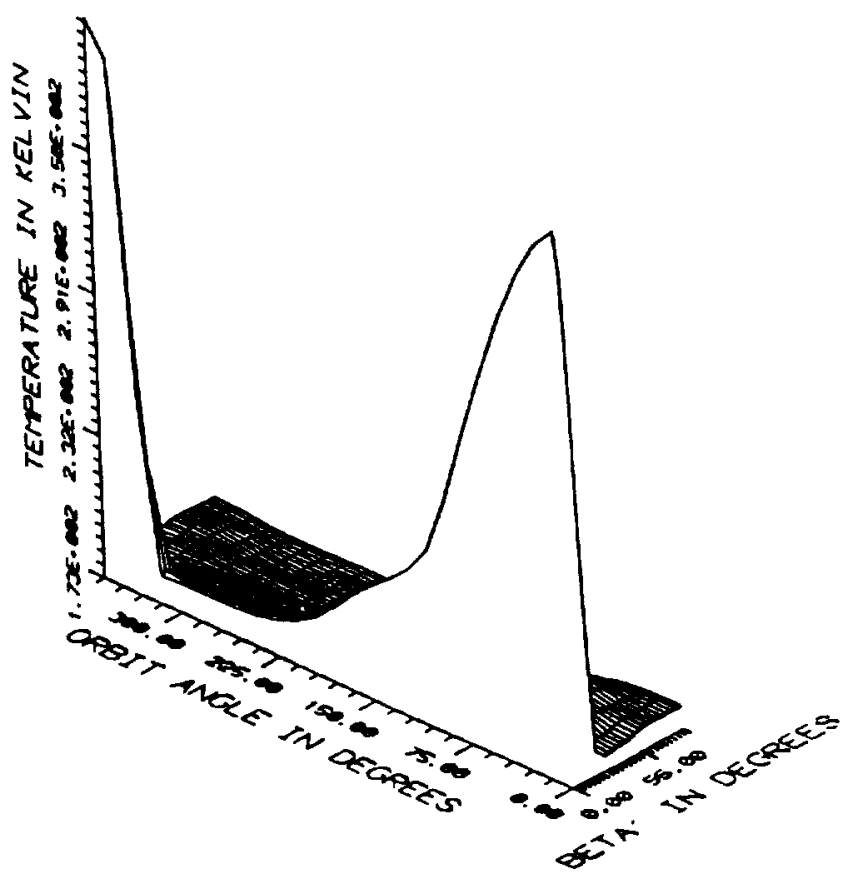

Figure 56. $X+$ Micro-Model Temperature Profile

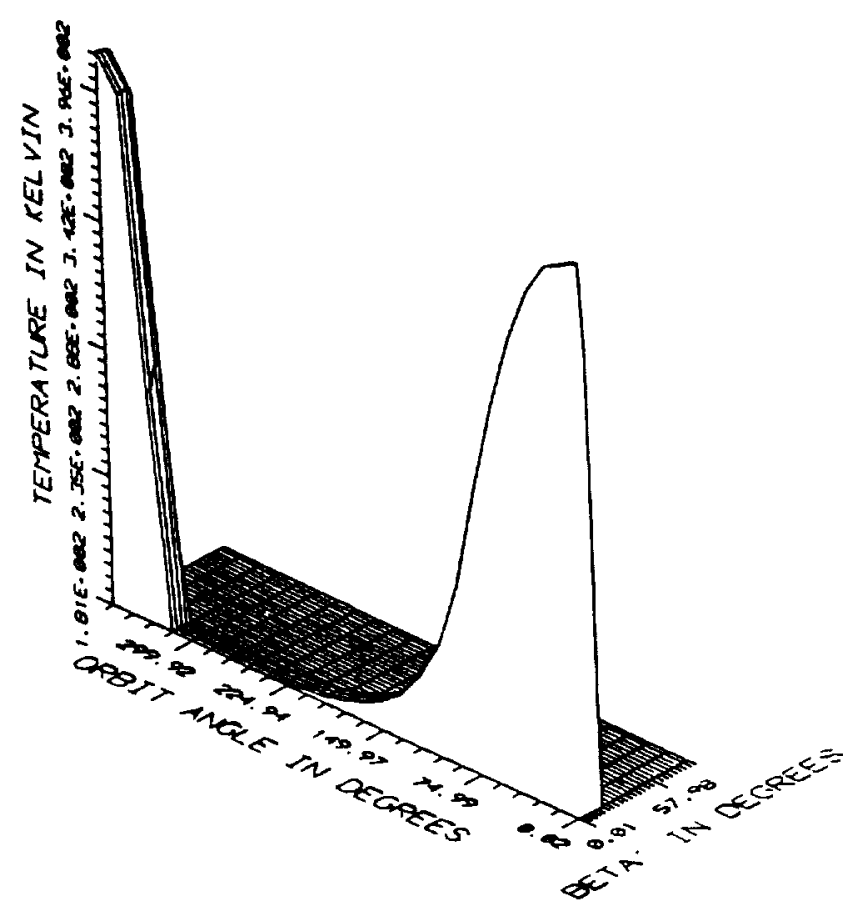

Figure 57. $X+$ Macro-Model Temperature Profile 


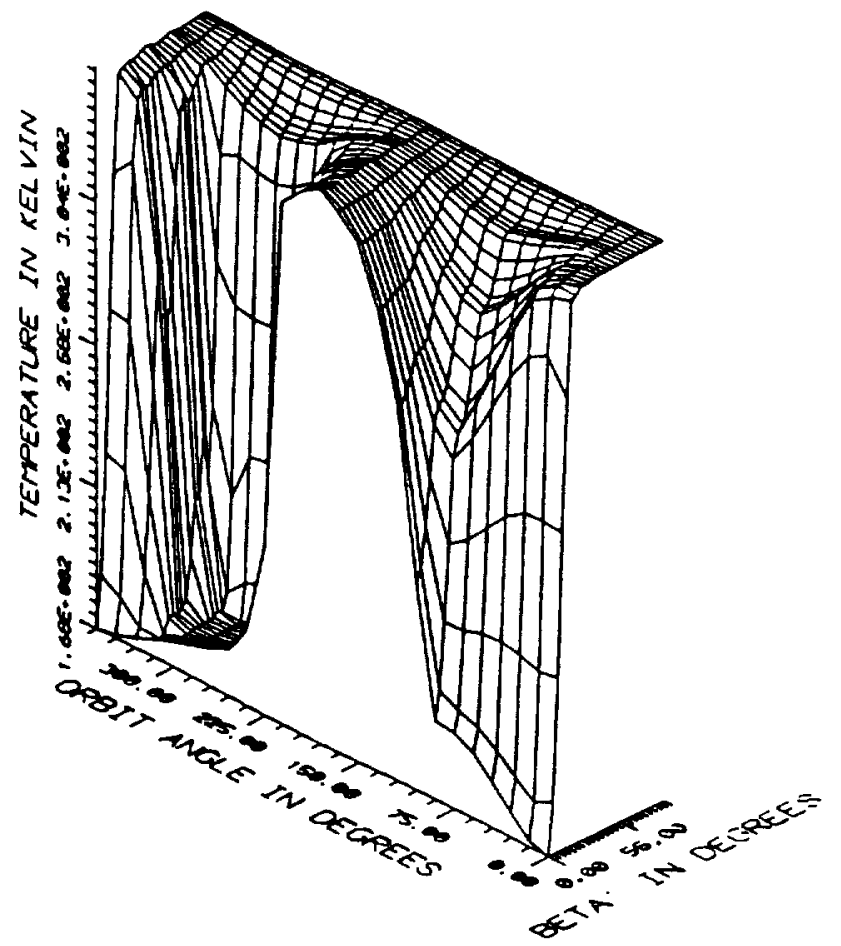

Figure 58. X-Micro-Model Temperature Profile

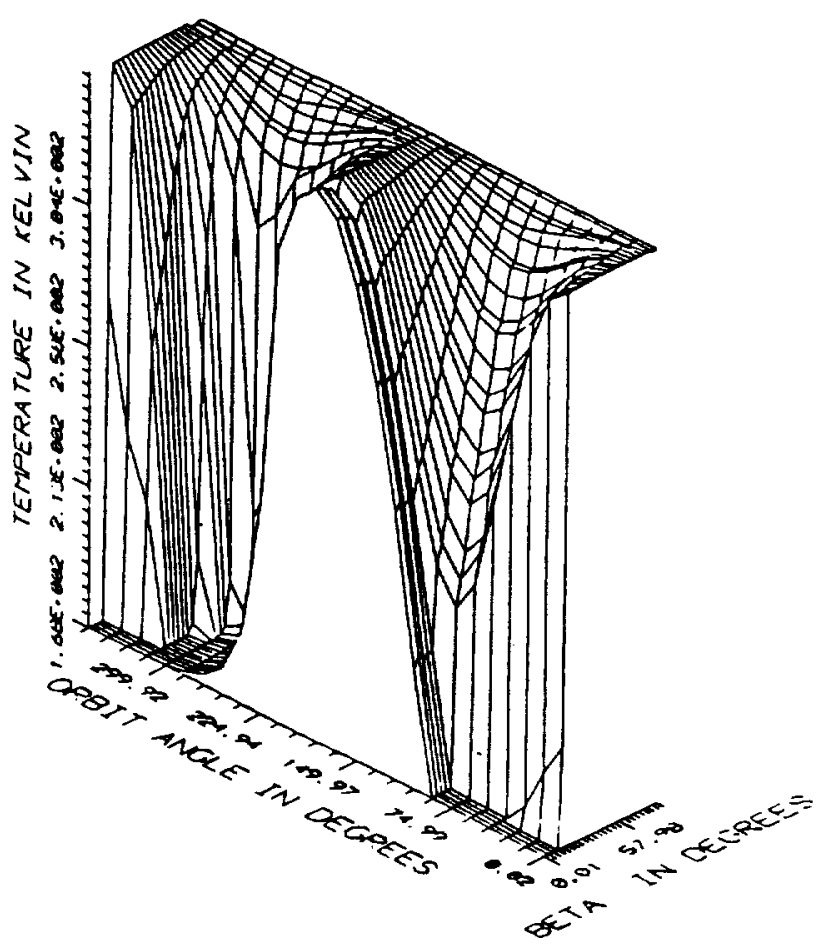

Figure 59. X-Macro-Model Temperature Profile 


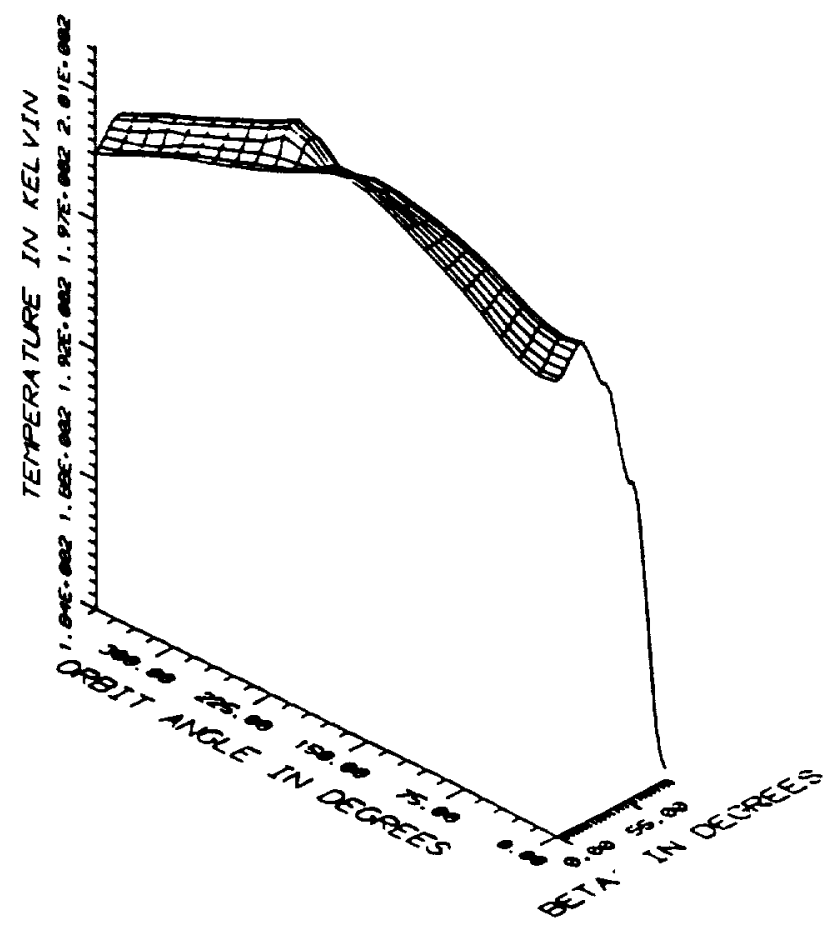

Figure 60. $Y+$ Micro-Model Temperature Profile

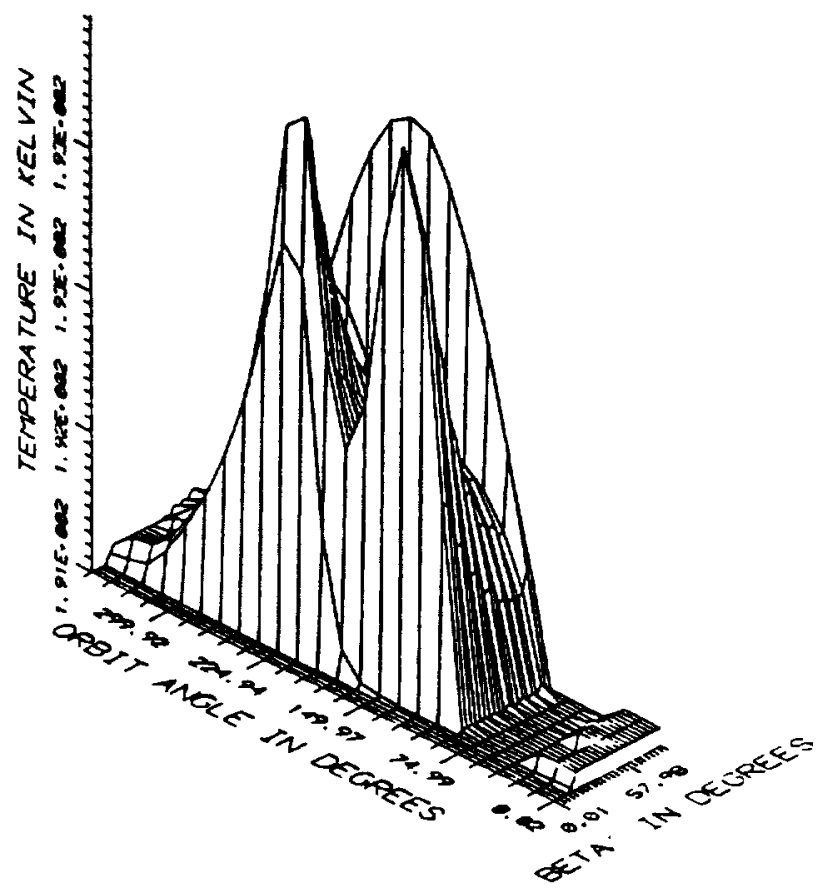

Figure 61. Y+ Macro-Model Temperature Profile 


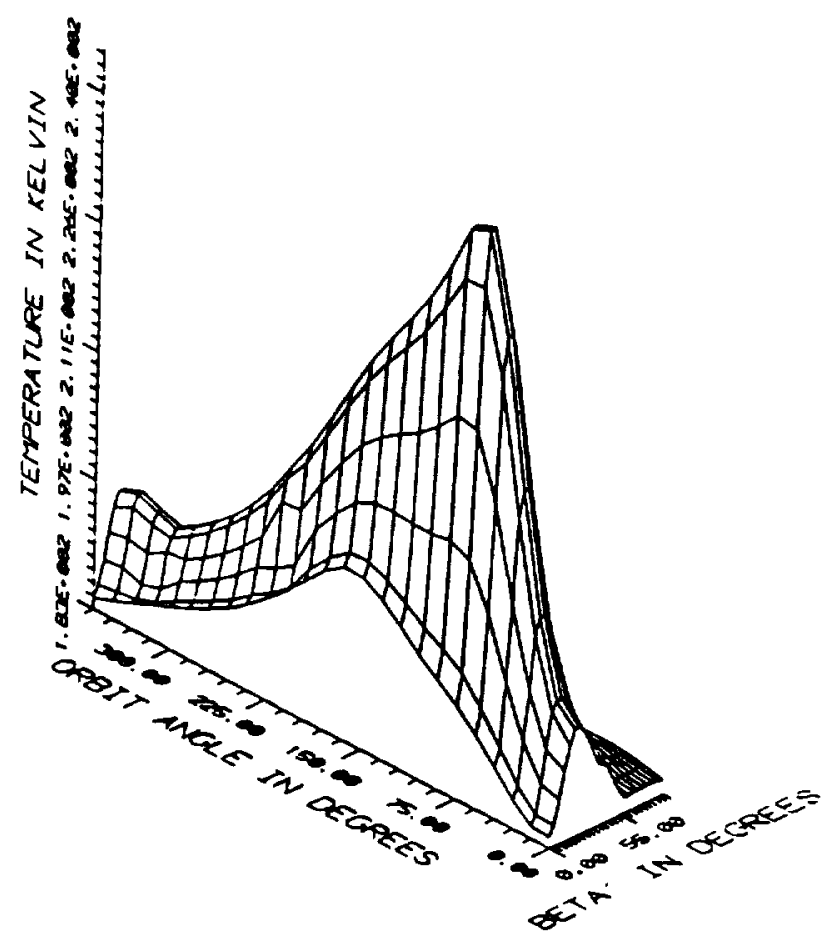

Figure 62. Y-Micro-Model Temperature Profile

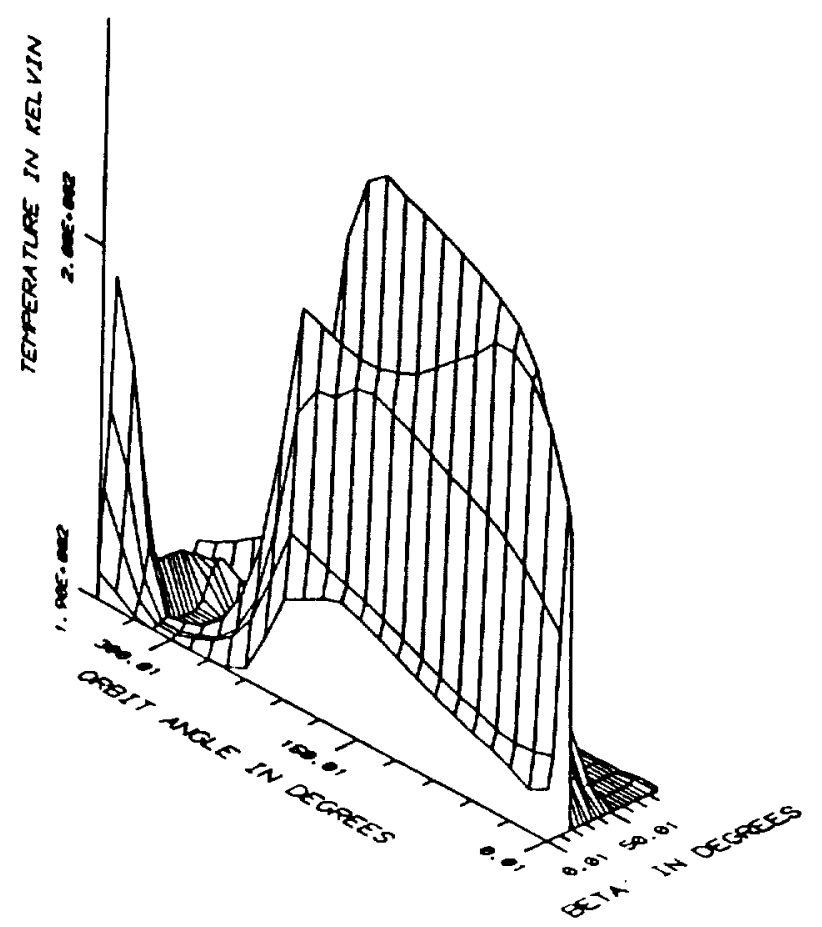

Figure 63. Y-Macro-Model Temperature Profile 


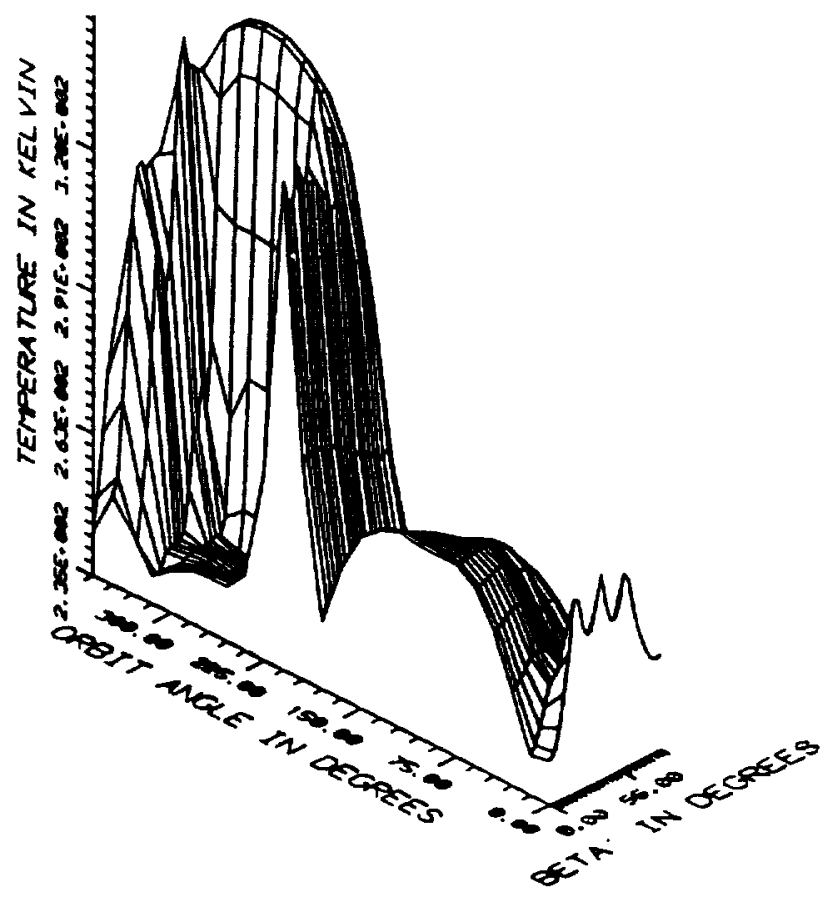

Figure 64. Z+ Micro-Model Temperature Profile

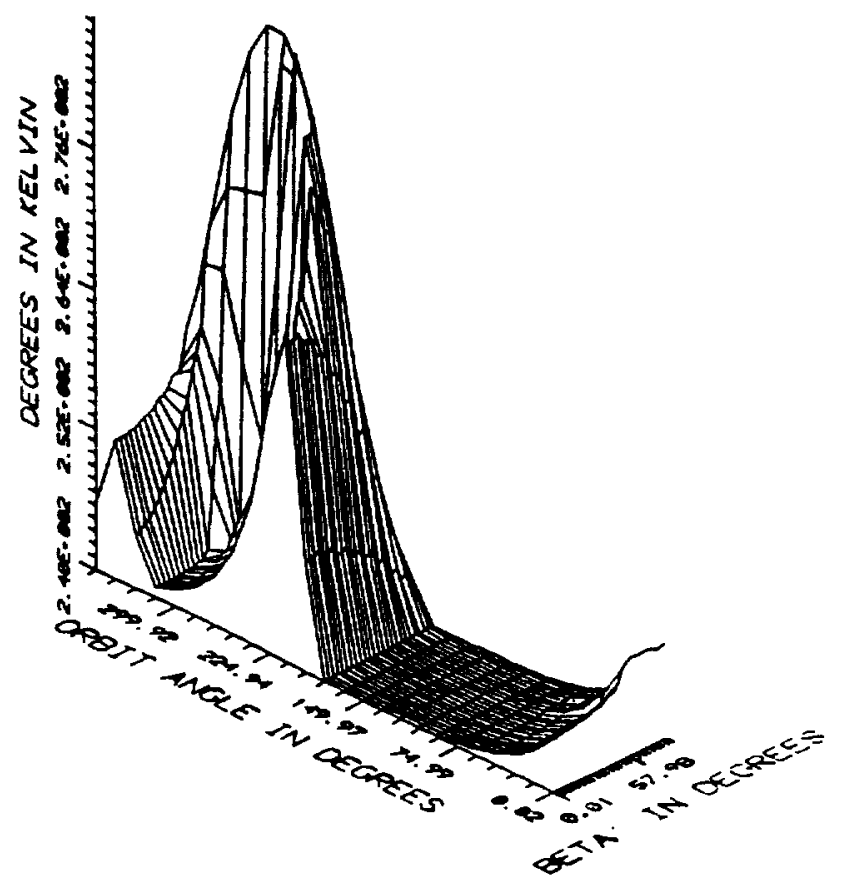

Figure 65. $Z+$ Macro-Model Temperature Profile 


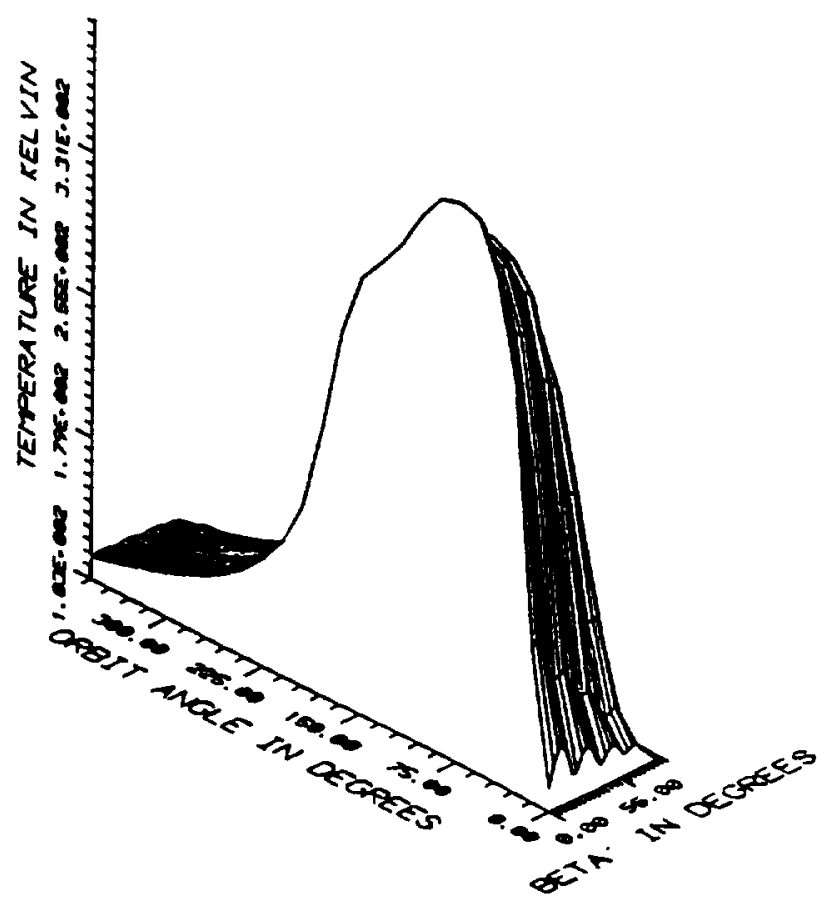

Figure 66. Z- Micro-Model Temperature Profile

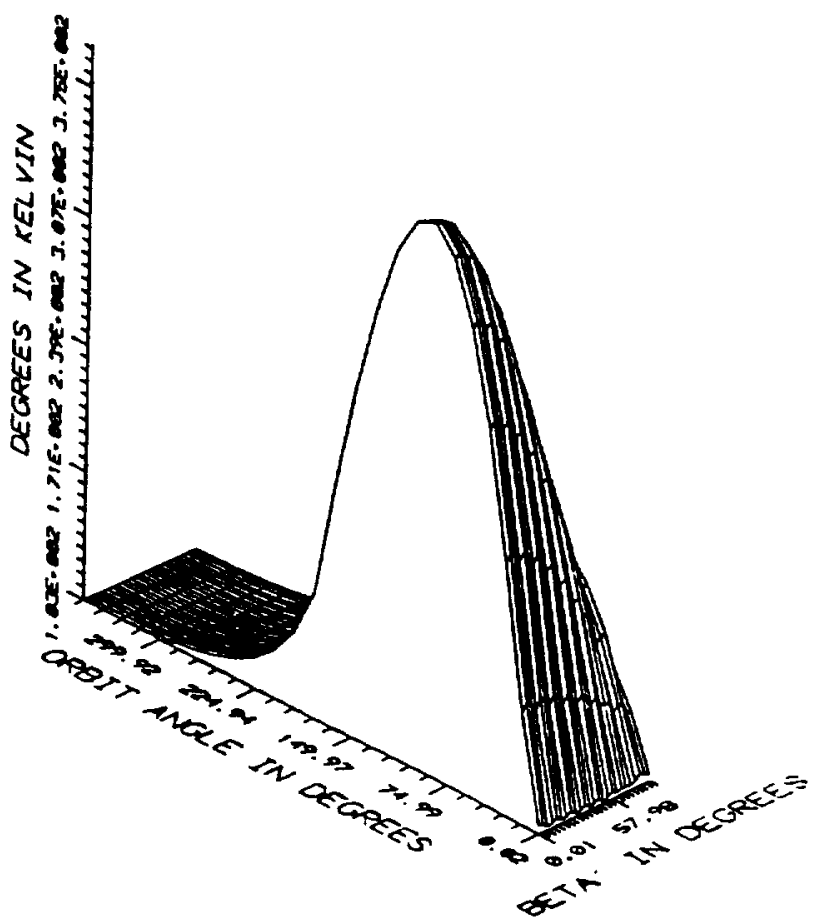

Figure 67. Z- Macro-Model Temperature Profile 


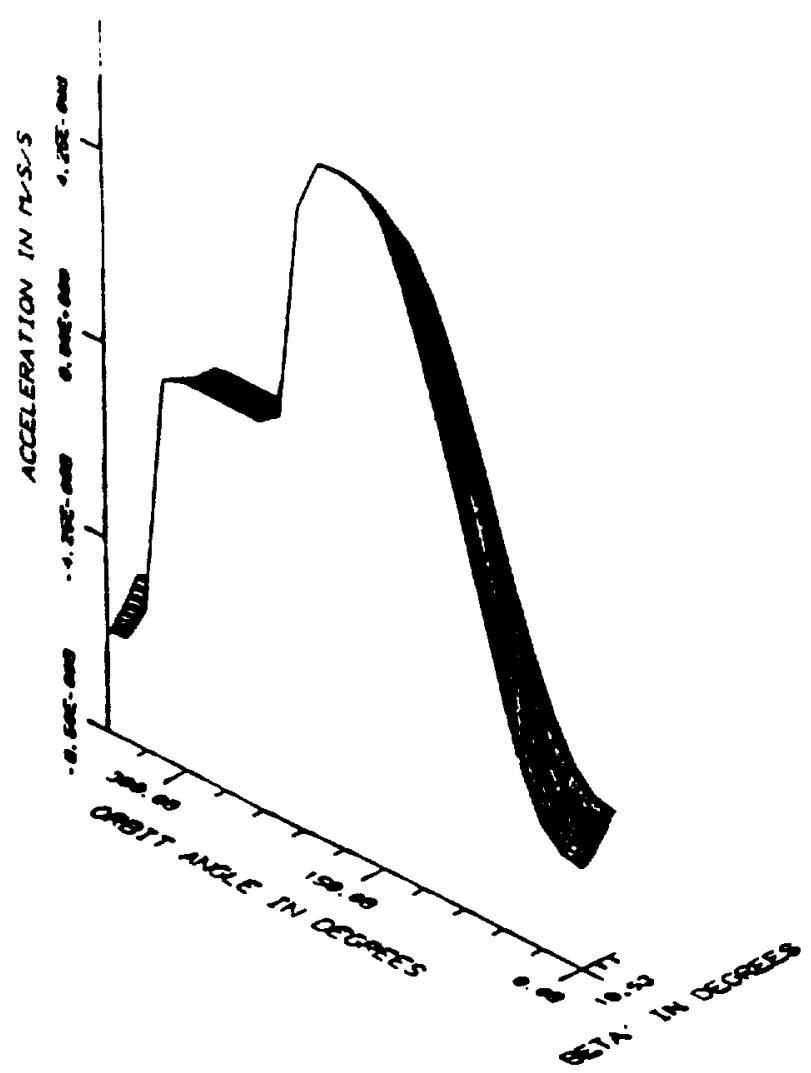

Figure 68. Billinear Interpolated Micro Model Solar Radiation Pressure Along. Track Acceleration. For $10.5^{\circ}$ to $36^{\circ} \mathrm{B}^{\prime}$

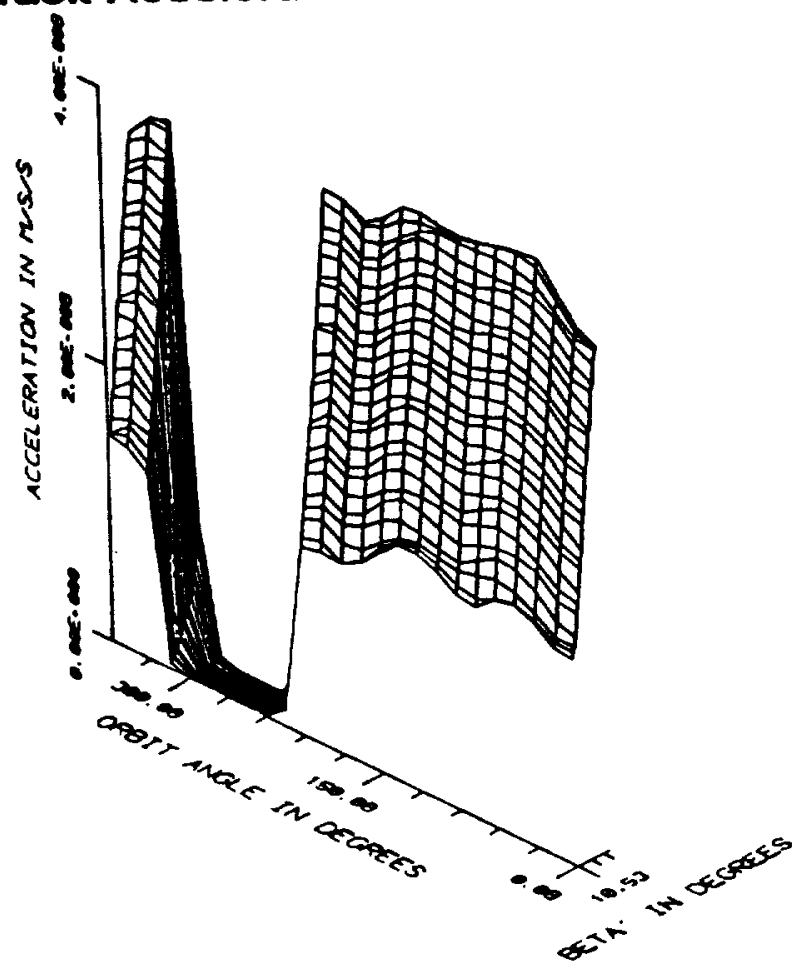

Figure 69. Bilinear Interpolated Micro Model Solar Radiation Pressure Cross. Track Acceleration. For $10.5^{\circ}$ to $36^{\circ} \mathrm{B}^{\prime}$ 


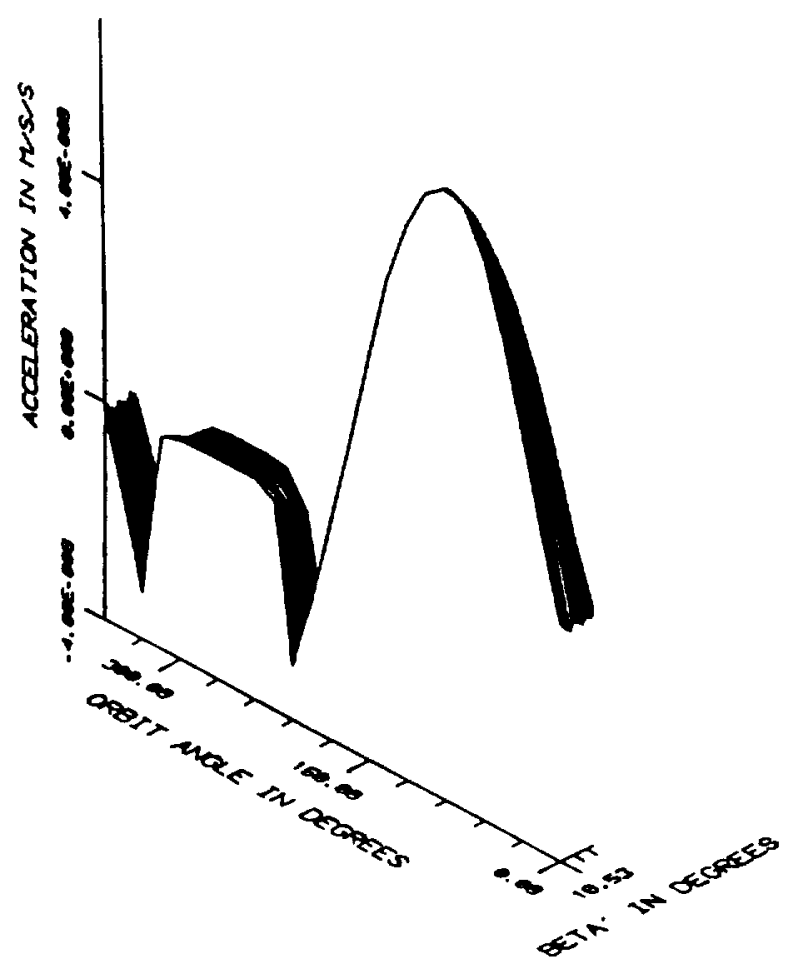

Figure 70. Bilinear Interpolated Micro Model Solar Radiation Pressure Radial Acceleration For $10.5^{\circ}$ to $36^{\circ} \mathrm{B}^{\prime}$

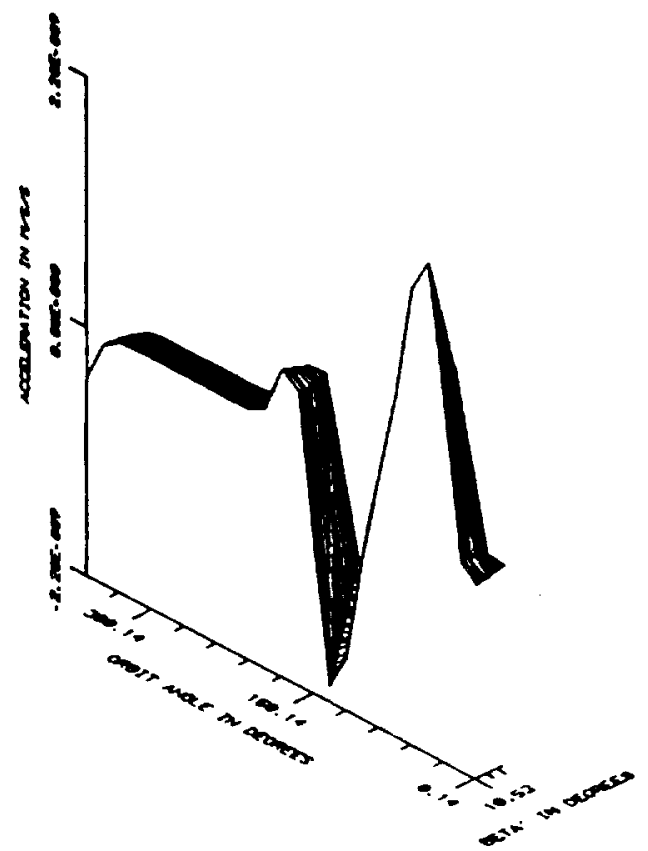

Figure 71. Bilinear Interpolated Micro Model Albedo Along Track Acceleration. For $10.5^{\circ}$ to $36^{\circ} \mathrm{B}^{\prime}$ 


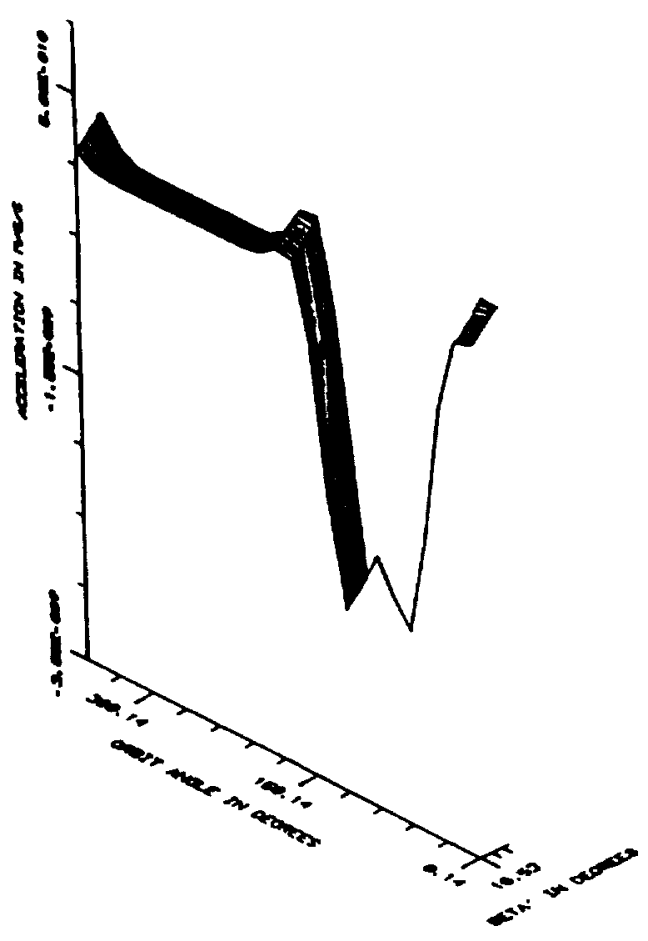

Figure 72. Bilinear Interpolated Micro Model Albedo Cross Track Acceleration. For $10.5^{\circ}$ to $36^{\circ} \mathrm{B}^{\prime}$

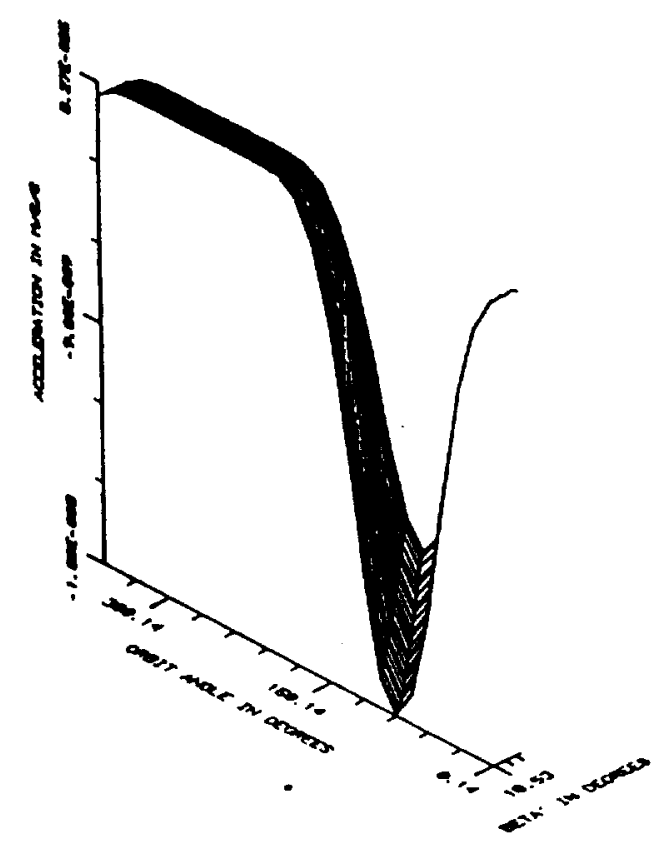

Figure 73. Bilinear Interpolated Micro Model Albedo Radial Acceleration. For $10.5^{\circ}$ to $36^{\circ} \mathrm{B}^{\prime}$

72 


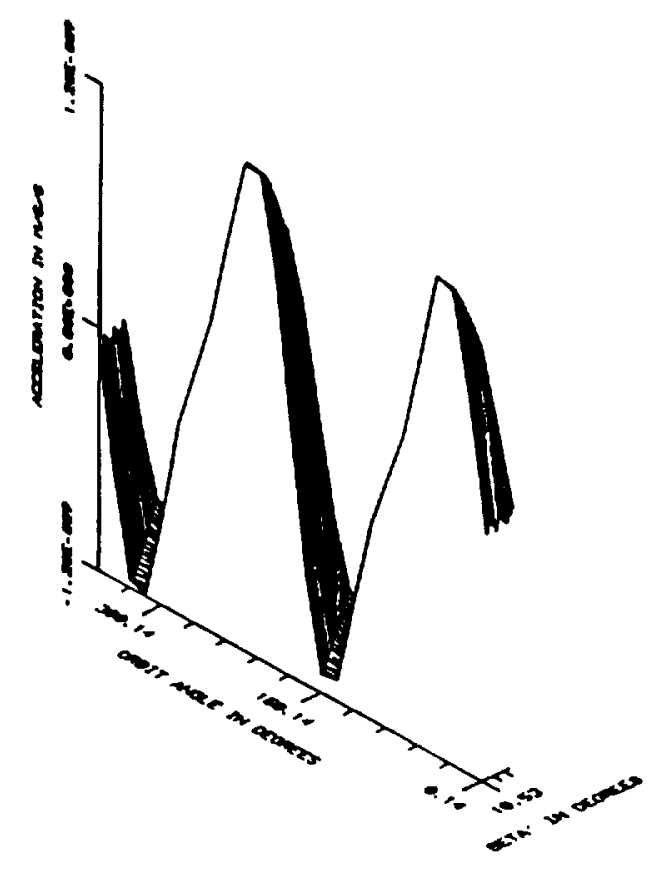

Figure 74. Bilinear Interpolated Micro Model IR Along Track Acceleration. For $10.5^{\circ}$ to $36^{\circ} B^{\prime}$

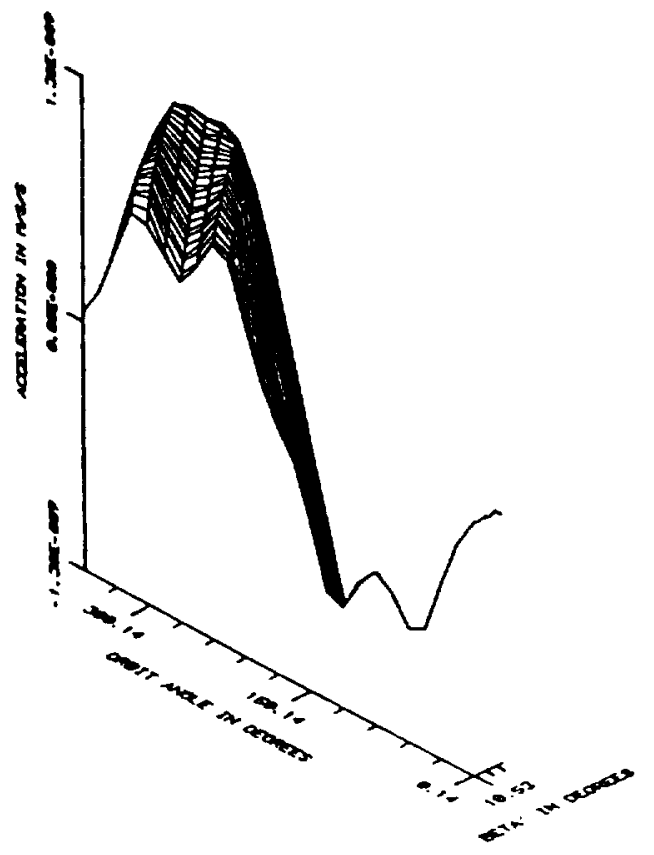

Figure 75. Bilinear Interpolated Micro Model IR Cross Track Acceleration. For $10.5^{\circ}$ to $36^{\circ} \mathrm{B}^{\prime}$ 


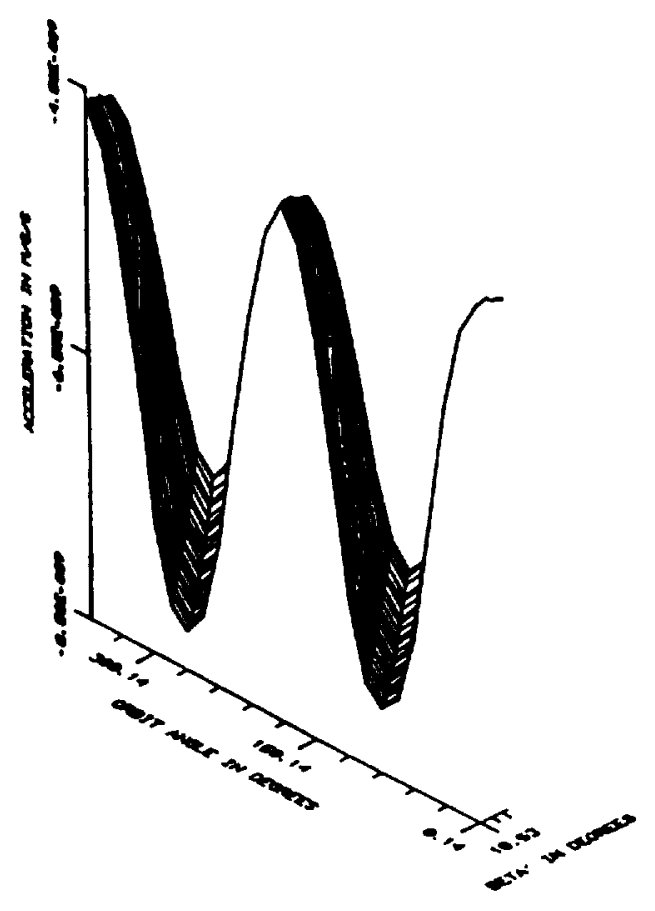

Figure 76. Bilinear Interpolated Micro Model IR Radial Acceleration. For $10.5^{\circ}$ to $36^{\circ} \mathrm{B}^{\prime}$

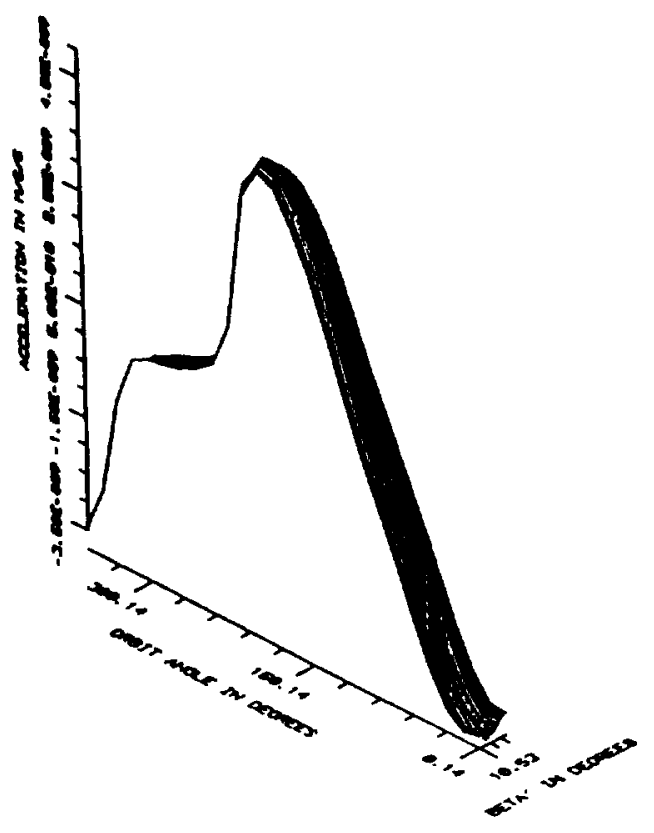

Figure 77. Bilinear Interpolated Micro Model Thermal Along Track Acceleration. For $10.5^{\circ}$ to $36^{\circ} B^{\prime}$ 


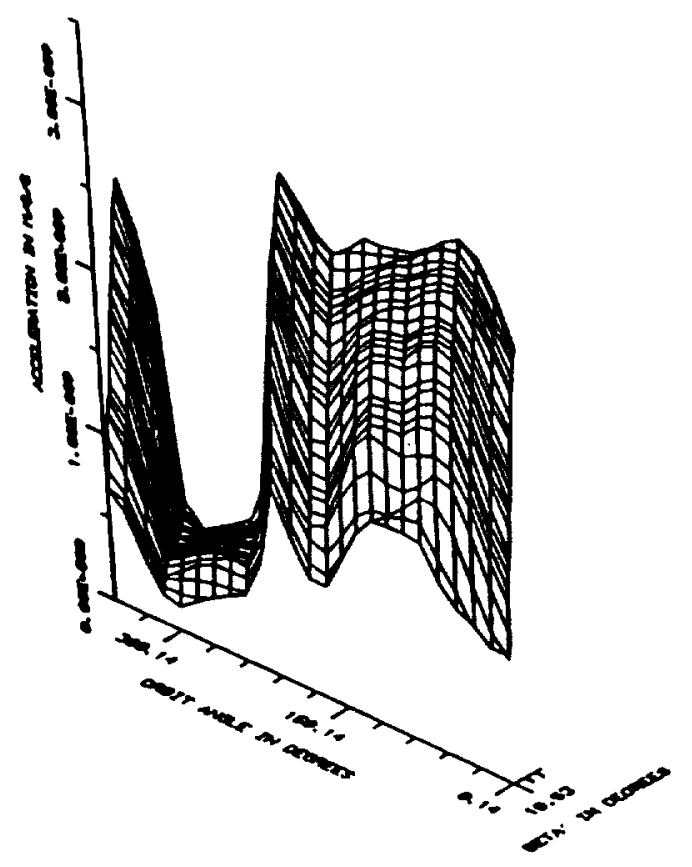

Figure 78. Bilinear Interpolated Micro Model Thermal Cross Track Acceleration. For $10.5^{\circ}$ to $36^{\circ} \mathrm{B}^{\prime}$

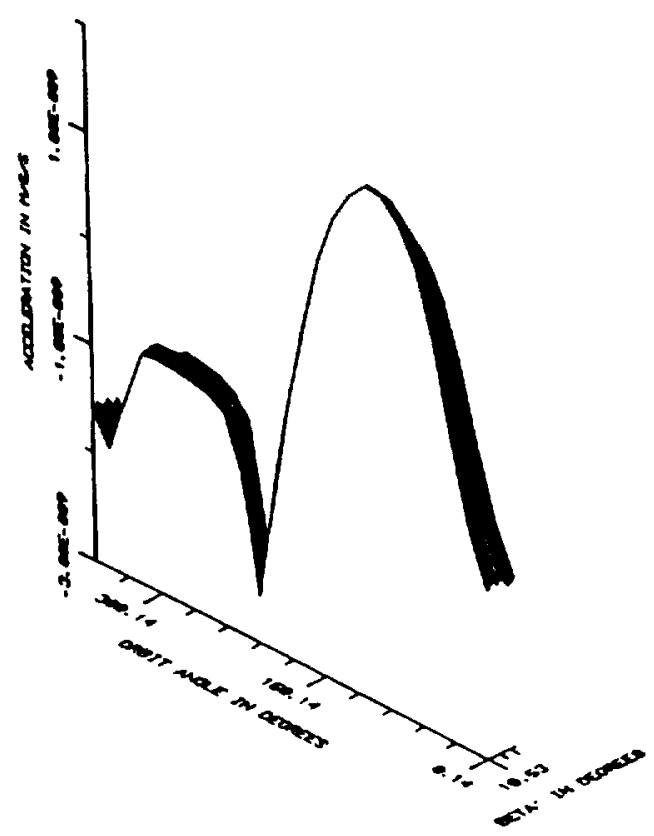

Figure 79. Bilinear Interpolated Micro Model Thermal Radial Acceleration. For $10.5^{\circ}$ to $36^{\circ} \mathrm{B}^{\prime}$ 


\section{REPORT DOCUMENTATION PAGE}

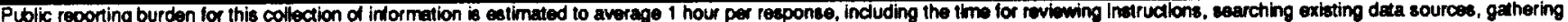
and maintaining the data needed, and complening and reviewing the collection of information. Send comments regarding this burden extimate of any other aspect of this collection of intormation, including suggestlons for reducing this bunden, to Wachington Headquarters Services, Directorate for Intormation Operations and Reports, 1215 Jefterson Davis Highway, Suke 1204, Anlington, VA 222024302, and to the Office of Managernent and Budget, Paperwork Reduction Project (0704-0188), Waahlngton, 0 C 20503.

\begin{tabular}{l|l|l|l} 
1. AGENCY USE ONLY (Leeve blenk) & 2. REPORT DATE & 3. REPORT TYPE AND DATES COVERED
\end{tabular} June 1992 Technical Memorandum

\section{TITLE AND SUBTITLE \\ Modeling Radiation Forces Acting on TOPEX/Poseidon}

for Precision Orbit Determination

\section{FUNDING NUMBERS}

6. AUTHOR(S)

J.A. Marshall, S.B. Luthcke, P.G. Antreasian, and G.W. Rosborough

7. PERFORMING ORGANIZATION NAME(S) AND ADDRESS(ES)

NASA-Goddard Space Flight Center

8. PERFORINING ORGANIZATION REPORT NUMBER

Greenbelt, Maryland 20771

$92 \mathrm{~B} 00089$

9. SPONSORINGANONITORING AGENCY NAME(S) AND ADDRESS(ES)

10. SPONSORINGMONITORING AGENCY REPORT NUMBER

National Aeronautics and Space Administration

Washington, D.C. 20546-0001

TM-104565

104544

\section{SUPPLEMENTARY NOTES}

J.A. Marshall: NASA-GSFC, Greenbelt, Maryland; S.B. Luthcke: Hughes STX Corporation, Lanham, Maryland; P.G. Antreasian and G.W. Rosborough: Department of Aerospace Engineering Sciences, University of Colorado, Boulder, Colorado.

12a. DISTRIBUTION/AVAILABIUTY STATEMENT

Unclassified - Unlimited

Subject Category 14 12b. DISTAIBUTION CODE

\section{ABSTRACT (Maximum 200 words)}

Geodetic satellites such as GEOSAT, SPOT, ERS-1, and TOPEX/Poseidon require accurate orbital computations to support the scientific data they collect. Until recently, gravity field mismodeling was the major source of error in precise orbit definition. However, with recent model improvements, accurate modeling of the nonconservative forces has become a significant concem. TOPEX/Poseidon mission requirements dictate that mismodeling of the nonconservative forces of solar radiation, Earth albedo and infrared re-radiation, and spacecraft thermal imbalances produce in combination no more than a 6-cm radial rms error over a 10-day period. This requires the development of nonconservative force models that take the satellite's complex geometry, attitude, and surface properties into account. For TOPEX/Poseidon, a "box-wing" satellite form has been investigated that models the satellite as a combination of flat plates arranged in a box shape with a connected solar array. The nonconservative forces acting on each of the eight surfaces are computed independently, yielding vector accelerations which are summed to compute the total aggregate effect on the satellite center-of-mass. In order to test the validity of this concept, "micro-models" based on finite element analysis of TOPEX/Poseidon have been used to generate acceleration histories in a wide variety of orbit orientations. These profiles are then compared to the boxwing model. The results of these simulations and their implication on the ability to precisely model the TOPEX/Poseidon orbit are discussed.

\section{SUBJECT TERMS}

15. NUMBER OF PAGES

Orbit Calculation, Geodesy, TOPEX/Poseidon, Solar Radiation, Earth Albedo, Spacecraft Thermal Properties, Nonconservative Forces, Finite Element Analysis, 75 Modeling

17. SECURITY CLASSIFICATION 1 18. SECURITY CLASSIFICATION 1 19. SECUAITY CLASSIFICA TION OF REPORT OF THIS PAGE

Unclassified

Unclassified
Unclassified OF ABSTRACT

\begin{tabular}{|l|} 
15. NUNBER OF PAGES \\
75 \\
\hline 16. PRICE CODE \\
$\begin{array}{l}\text { 20. UMITATION OF AB- } \\
\text { STRACT } \\
\text { Unlimited }\end{array}$ \\
\hline
\end{tabular}

